\title{
CODEX weak lensing mass catalogue and implications on the mass-richness relation
}

Kiiveri, K.

2021-03

Kiiveri , K , Gruen , D , Finoguenov , A, Erben , T , van Waerbeke , L , Rykoff , E , Miller , L , Hagstotz , S, Dupke , R , Henry , J P , Kneib , J-P , Gozaliasl , G, Kirkpatrick , C C , Cibirka , N , Clerc , N , Costanzi , M , Cypriano , E S, Rozo , E, Shan , H, Spinelli , P , Valiviita , J \& Weller , J 2021, ' CODEX weak lensing mass catalogue and implications on the mass-richness relation ' , Monthly Notices of the Royal Astronomical Society, vol. 502 , no. 1 , pp. 1494-1526 . https://doi.org/10.1093/mnras/staa3936

http://hdl.handle.net/10138/336506

https://doi.org/10.1093/mnras/staa3936

acceptedVersion

Downloaded from Helda, University of Helsinki institutional repository.

This is an electronic reprint of the original article.

This reprint may differ from the original in pagination and typographic detail.

Please cite the original version. 


\title{
CODEX Weak Lensing Mass Catalogue and implications on the mass-richness relation
}

\author{
K. Kiiveri ${ }^{1,2}$, D. Gruen ${ }^{3,4}$, A. Finoguenov ${ }^{1}$, T. Erben $^{5}$, L. van Waerbeke ${ }^{6}$, E. Rykoff ${ }^{3,4}$, \\ L. Miller ${ }^{7}$, S. Hagstotz ${ }^{8}$, R. Dupke ${ }^{9}$, J. Patrick Henry ${ }^{10}$, J-P. Kneib ${ }^{11}$, G. Gozaliasl ${ }^{1,2}$, \\ C. C. Kirkpatrick ${ }^{1,2}$, N. Cibirka ${ }^{13,14}$, N. Clerc ${ }^{15}$, M. Costanzi ${ }^{16}$, E. S. Cypriano ${ }^{13}$, \\ E. Rozo ${ }^{17}$, H. Shan ${ }^{18}$, P. Spinellii ${ }^{19}$, J. Valiviita ${ }^{2,20}$, J. Weller ${ }^{14,21}$ \\ ${ }^{1}$ Department of Physics, University of Helsinki, PO Box 64, FI-00014 Helsinki, Finland \\ ${ }^{2}$ Helsinki Institute of Physics, Gustaf Hällströmin katu 2, University of Helsinki, Helsinki, Finland \\ ${ }^{3}$ Kavli Institute for Particle Astrophysics \& Cosmology, P. O. Box 2450, Stanford University, Stanford, CA 94305, USA \\ ${ }^{4}$ SLAC National Accelerator Laboratory, Menlo Park, CA 94025, USA \\ ${ }^{5}$ Argelander-Institut für Astronomie, Auf dem Hügel 71, D-53121 Bonn, German \\ ${ }^{6}$ Department of Physics and Astronomy, University of British Columbia, 6224 Agricultural road, Vancouver, BC V6T 1Z1, Canada \\ ${ }^{7}$ Department of Physics, Oxford University, Keble Road, Oxford OX1 3RH, UK \\ ${ }^{8}$ Oskar Klein Centre, Department of Physics, Stockholm University, AlbaNova University Centre, SE 10691 Stockholm, Sweden \\ ${ }^{9}$ Observatório Nacional, Rua Gal. José Cristino, 20921-400, Rio de Janeiro, Brasil \\ Department of Astronomy, University of Michigan, 311 West Hall 1085 South University Ave. Ann Arbor, MI 48109-1107, USA \\ Department of Physics and Astronomy, University of Alabama, Box 870324, Tuscaloosa, AL 35487, USA \\ Eureka Scientific Inc., 2452 Delmer St. Suite 100, Oakland, CA 94602, USA \\ ${ }^{10}$ Institute for Astronomy, 2680 Woodlawn Drive, Honolulu, HI 96822, USA \\ ${ }^{11}$ Laboratoire d'Astrophysique, Ecole Polytechnique Fédérale de Lausanne (EPFL), Observatoire de Sauverny, CH-1290 Versoix, Switzerland \\ Aix Marseille Université, CNRS, LAM (Laboratoire d'Astrophysique de Marseille) UMR 7326, 13388, Marseille, France \\ ${ }^{13}$ Instituto de Astronomia, Geofísica e Ciências Atmosféricas, Universidade de São Paulo, Brasil \\ ${ }^{14}$ Universitäts-Sternwarte, Fakultät für Physik, Ludwig-Maximilians-Universität München, Scheinerstraße 1, D-81679 München, Germany \\ ${ }^{15}$ CNRS, IRAP, 9 Av. colonel Roche, BP 44346, F-31028; Université de Toulouse, UPS-OMP, Toulouse, France \\ ${ }^{16}$ INAF-Osservatorio Astronomico di Trieste, Via G. B Tiepolo 11, I-34143; IFPU-Institute for Fundamental Physics of the Universe, Via Beirut 2, 34014 Trieste, Italy \\ ${ }^{17}$ Department of Physics, University of Arizona, 1118 E. Fourth St, Tucson, AZ 85721, USA \\ ${ }^{18}$ Shanghai Astronomical Observatory (SHAO), Nandan Road 80, Shanghai 200030, China \\ ${ }^{19}$ Museu de Astronomia e Ciências Afins (MAST), Rua General Bruce 586, 20921-030 Rio de Janeiro, Brasil \\ ${ }^{20}$ Department of Physics, P.O.Box 35 (YFL), FI-40014 University of Jyväskylä, Finland \\ ${ }^{21}$ Excellence Cluster Origins, Boltzmannstraße 2, D-85748 Garching, Germany
}

Accepted XXX. Received YYY; in original form ZZZ

\begin{abstract}
The COnstrain Dark Energy with X-ray clusters (CODEX) sample contains the largest flux limited sample of X-ray clusters at $0.35<z<0.65$. It was selected from ROSAT data in the 10,000 square degrees of overlap with BOSS, mapping a total number of 2770 high-z galaxy clusters. We present here the full results of the CFHT CODEX program on cluster mass measurement, including a reanalysis of CFHTLS Wide data, with 25 individual lensingconstrained cluster masses. We employ lensfit shape measurement and perform a conservative colour-space selection and weighting of background galaxies. Using the combination of shape noise and an analytic covariance for intrinsic variations of cluster profiles at fixed mass due to large scale structure, miscentring, and variations in concentration and ellipticity, we determine the likelihood of the observed shear signal as a function of true mass for each cluster. We combine 25 individual cluster mass likelihoods in a Bayesian hierarchical scheme with the inclusion of optical and X-ray selection functions to derive constraints on the slope $\alpha$, normalization $\beta$, and scatter $\sigma_{\ln \lambda \mid \mu}$ of our richness-mass scaling relation model in logspace: $\langle\ln \lambda \mid \mu\rangle=\alpha \mu+\beta$, with $\mu=\ln \left(M_{200 c} / M_{\text {piv }}\right)$, and $M_{\text {piv }}=10^{14.81} M_{\odot}$. We find a slope $\alpha=0.49_{-0.15}^{+0.20}$, normalization $\exp (\beta)=84.0_{-14.8}^{+9.2}$ and $\sigma_{\ln \lambda \mid \mu}=0.17_{-0.09}^{+0.13}$ using CFHT richness estimates. In comparison to other weak lensing richness-mass relations, we find the normalization of the richness statistically agreeing with the normalization of other scaling relations from a broad redshift range $(0.0<z<0.65)$ and with different cluster selection (X-ray, Sunyaev-Zeldovich, and optical).
\end{abstract}

Key words: galaxy: clusters - cosmology: observations - gravitational lensing: weak 


\section{$2 \quad$ K. Kiiveri et al.}

\section{INTRODUCTION}

Clusters of galaxies represent the end product of hierarchical structure formation. They play a key role in understanding the cosmological interplay of dark matter and dark energy. Their number density, baryonic content, and their growth are sensitive probes of cosmological parameters, such as the mean dark matter and dark energy density $\Omega_{\mathrm{m}}$ and $\Omega_{\Lambda}$, the dark energy equation of state parameter $w$ and the normalization of the matter power spectrum $\sigma_{8}$ (see Allen et al. 2011 for a recent review). The idea of using cluster counts to probe cosmology is based on the halo mass function, which predicts their number density as a function of mass, redshift and cosmological parameters (see e.g. Press \& Schechter 1974; Sheth $\&$ Tormen 1999; Tinker et al. 2008). The observational task consists of obtaining an ensemble of galaxy clusters with an observable that correlates with their true mass and a well defined selection function. In recent years a number of multiwavelength, deep, and wide observations and surveys have been conducted which allow to detect galaxy clusters with a high signal-to-noise ratio $(\mathrm{S} / \mathrm{N})$ out to high redshifts (e.g., $z \sim 2.5$ ). Observations are based on properties of baryonic origin, among them the number count of red galaxies (called richness, see e.g. Gladders \& Yee 2005; Koester et al. 2007; Rykoff et al. 2014) or the inverse Compton scattering of cosmic microwave photons on the hot intra-cluster gas (the Sunyaev \& Zeldovich 1980 effect, see Bleem et al. 2015 and Planck Collaboration et al. 2016 for the latest observational results). Another approach is to select a galaxy cluster sample from X-ray observations (see e.g. Ebeling et al. 1998; Böhringer et al. 2004; Ebeling et al. 2010; Gozaliasl et al. 2014, 2019). However, hydrodynamical simulations have shown that even for excellently measured X-ray observables with small intrinsic scatter at fixed mass and dynamically relaxed clusters at optimal measurement radii $\left(r \sim r_{2500}\right)$, non-thermal pressure support from residual gas bulk motion and other processes are expected to bias the hydrostatic X-ray mass estimates down by up to 5-10 per cent (see Nagai et al. 2007; Rasia et al. 2012), which represents the currently dominant systematic uncertainty in constraining cosmology from X-ray cluster samples (see Henry et al. 2009; Vikhlinin et al. 2009; Mantz et al. 2010; Rozo et al. 2010; Benson et al. 2013; Mantz et al. 2015).

For this reason, the idea of absolute calibration of the mass scale of large cluster samples by weak gravitational cluster lensing (see e.g. Hoekstra 2007; Marrone et al. 2012; Gruen et al. 2014; von der Linden et al. 2014a,b; Melchior et al. 2016; Herbonnet et al. 2019) has gained traction over the last years. Weak gravitational lensing is sensitive to the entire gravitational matter and is therefore mostly free of systematic uncertainty that relates to the more complex interaction of baryons.

However, weak lensing mass measurements for individual clusters are inherently quite noisy, as the measured ellipticities of background galaxies do not only depend on the gravitational shear induced by the analysed galaxy cluster but also on the quite broad intrinsic ellipticity distribution, and on the gravitational imprint of all matter along the line of sight, including unrelated projected structure (see e.g. Hoekstra 2001, 2003; Spinelli et al. 2012). On top of this, at fixed true mass the density profiles of clusters intrinsically vary, causing additional scatter in weak lensing mass estimation (Becker \& Kravtsov 2011; Gruen et al. 2011, 2015). For this reason, relatively large samples of galaxy clusters need to be investigated to statistically meet the calibration requirements of cosmology.

Even with large samples of clusters and sufficiently deep optical data to measure the shapes of numerous background galaxies, several systematic uncertainties limit the power of weak lensing mass calibration. Firstly, shape measurement algorithms commonly recover the amplitude of gravitational shear only with a one to several per-cent level multiplicative bias (e.g. Mandelbaum et al. 2015; Jarvis et al. 2016; Fenech Conti et al. 2017, but see the recent advances of Huff \& Mandelbaum 2017; Sheldon \& Huff 2017). Secondly, the amplitude of the weak lensing signal does not only depend on the cluster mass, but also on the geometric configuration between observer, lens and background objects, more specific on their angular diameter distances among the observer, lens, and source. For interpreting the shear signal, additional photometric data are required to obtain the necessary distance information by photometric redshifts (Lerchster et al. 2011; Gruen et al. 2013), colour cuts or distance estimates by colour-magnitude properties (Gruen et al. 2014; Cibirka et al. 2016). All these methods suffer from systematic uncertainties (see e.g. Applegate et al. 2014; Gruen \& Brimioulle 2016) that translate to systematic errors in cluster masses. On a related note, cluster member galaxies can enter the photometrically selected background galaxy sample and lower the observed gravitational shear signal (see e.g. Sheldon et al. 2004; Gruen et al. 2014; Melchior et al. 2016 for different methods of estimating and correcting the impact of this). Finally, a mismatch between the fitted density profile and the underlying true mean profile of clusters at a given mass (including the miscentring of clusters relative to the assumed positions in the lensing analysis) can cause significant uncertainty in weak lensing cluster mass estimates (see e.g. Melchior et al. 2016).

In this COnstrain Dark Energy with X-ray clusters (CODEX) study we present weak lensing mass analysis for a total of 25 galaxy clusters. The initial CODEX sample of 407 clusters, from which the main lensing sample is obtained, is cut at $0.35<z<0.65$ with $\lambda \geqslant 60$ with $\mathrm{X}$-ray based selection function. To this end, we also develop new methods to provide a full likelihood of the lensing signal as a function of individual cluster mass, and carefully characterize the systematic uncertainty.

This paper is structured as follows. In section 2 we present the data and analysis, including data reduction, photometric processing, richness estimation, shape measurement and mass likelihood. In section 3 we describe our Hierarchical Bayesian model, which we use to estimate richness-mass relation. In section 4 we apply the Hierarchical Bayesian model to find the richness-mass relation of all 25 clusters in the weak lensing mass catalog. In section 5 we present our results of the Bayesian analysis, and in section 6 we summarize and conclude. In the Appendix, we detail our systematic uncertainties, fields with incomplete colour information, and present weak lensing mass measurements for 32 clusters excluded from the richness-mass calibration.

We adopt a concordance $\Lambda$ CDM cosmology and WMAP7 results (Komatsu et al. 2011) with $\Omega_{\mathrm{m}}=0.27, \Omega_{\Lambda}=0.73$ and $H_{0}=70$ $\mathrm{km} \mathrm{s}^{-1} \mathrm{Mpc}^{-1}$. The halo mass of galaxy clusters in this study corresponds to $M_{200 c}$, defined as the mass within radius $r_{200 c}$, the radius in which the mass and concentration definitions is taken to be 200 times the critical density of the Universe $\left(\rho_{\mathrm{c}}\right)$.

\section{DATA AND ANALYSIS}

\subsection{Cluster catalogue}

The CODEX sample was initially selected by a $4 \sigma$ photon excess inside a wavelet aperture in the overlap of the ROSAT All-Sky Survey (RASS, Voges et al. 1999) with the Sloan Digital Sky Survey (SDSS). We use RASS photon images and search for X-ray 
sources on scales from 1.5 arcmin to 6 arcmin using wavelets. Any source detected is considered as a cluster candidate and enters the redMaPPer code (see Rykoff et al. 2014 and subsection 2.4), which associates an optical counterpart for each source and reports its richness and redshift. For this sample, we consider a high threshold of richness 60 and redshifts above 0.35 , which yields the sample of most massive X-ray selected high-z clusters, for which we seek to perform a weak lensing calibration. While other X-ray source catalogues using RASS data exist (e.g. Boller et al. 2016), the advantage of our approach consists of performing detailed modelling of the cluster selection function using our detection pipeline, which takes into account the RASS sensitivity as a function of sky position, Galactic absorption, and cluster detectability as a function of mass and redshift. Availability of such a selection function enables precise modelling of the cluster appearance in the catalog, critically important for the Bayesian modelling of the scaling relations.

At the positions of these overdensities, the redMaPPer algorithm is run to extract estimates of photometric redshift, richness, and a refined position and ROSAT X-ray flux estimate. For more details on the catalog construction, see Clerc et al. (2016), Cibirka et al. (2016) and Finoguenov et al. (2020).

The initial sample of 407 clusters is selected by the richness $\lambda_{\mathrm{RM} \text {,SDSS }}$ and redshift $z_{\mathrm{RM}}$,SDSS estimated from the redMaPPer run on SDSS photometric catalogues, cut at $\lambda_{\mathrm{RM}}$,SDSS $\geqslant 60$ and $0.35<z_{\text {RM }}$ SDSS $<0.65$. A subsample of the initial sample was chosen as a weak lensing follow-up with CFHT (Canada-FranceHawaii Telescope) designed to calibrate richness-mass relation for this survey. This deeper CFHT survey of 36 clusters, that we call S-I, falls into the CFHT Legacy Survey ${ }^{1}$ (CFHTLS) footprint, and is selected only by observability. To have an optically clean sample without missing data in CFHT richness or weak lensing mass, we exclude a total of 11 clusters, and define the remaining 25 cluster sample as our main lensing sample. The main lensing sample of 25 clusters is listed in Table 1. The excluded clusters of S-I are described in section 4, and listed in the Appendix Table C1.

Since weak lensing analysis requires precise knowledge of the cluster redshift, for 20 clusters without spectroscopic redshifts in $\mathrm{S}-\mathrm{I}$, we targeted red-sequence member galaxies for spectroscopy. The clusters observed as a part of the CFHT program, are targeted by several Nordic Optical Telescope (NOT) programs (PI A. Finoguenov, 48-025, 52-026, 53-020, 51-034). Each cluster is observed in multi-object spectroscopy mode, targeting $\sim 20$ member galaxies including Brightest Cluster Galaxies (BCGs) and having spectral resolving power of $\sim 500$. The typical exposure per mask is $2700 \mathrm{~s}$ with a grism that provides wavelength coverage between approximately $400-700 \mathrm{~nm}$. The average seeing over the four programmes is near 1 arcsec. Because we are solely interested in the redshift of the $\mathrm{Ca} \mathrm{H}+\mathrm{K}$ lines, only wavelength calibration frames are additionally obtained. Standard IRAF7 packages are used in the data reduction, spectra extraction and wavelength calibration process. The redshifts are determined finally using RVIDLINES to measure the positions of the two calcium lines for a weighted average fit. The acquired spectroscopic cluster redshifts for the weak lensing sample are listed in Table 1, along with X-ray observables, richness estimates, and available photometric data.

\subsection{Imaging data and data reduction}

This study comprises imaging data covering 34 pointings centred on CODEX clusters observed with the wide field optical camera MegaCam (Boulade et al. 2003) at the CFHT. For 28 of these pointings full colour information of filters $u$.MP9301, $g$.MP9401, $r$.MP9601, $i$.MP9702, z.MP9801 is available. All considered pointings possess $i$-band information. A summary of the imaging data of S-I can be seen in Appendix Table D1.

A detailed description of the data reduction can be found in Cibirka et al. (2016). We only give a brief overview here.

We process the CODEX data using the algorithms and processing pipelines (THELI) developed within the CFHTLS-Archive Research Survey (CARS, see Erben et al. 2009, 2005; Schirmer 2013) and CFHT Lensing Survey ${ }^{2}$, (CFHTLenS, see Erben et al. 2013; Heymans et al. 2012).

Starting point is the CODEX data, preprocessed with Elixir, available at the Canadian Astronomical Data Centre ${ }^{3}$ (CADC). The Elixir preprocessing removes the entire instrumental signature from the raw data and provides all necessary photometric calibration information.

The final data reduction comprises deselection of damaged raw images or images of low quality, astrometric and relative photometric calibration using scamp 4 (Bertin et al. 2002), coaddition of the final reduced single frames with swarp $^{5}$ and creation of image masks by running the automasktool ${ }^{6}$ (Dietrich et al. 2007) to indicate photometrically defective areas (satellite and asteroid tracks, bright, saturated stars and areas which would influence the analysis of faint background sources).

\subsection{Photometric catalogue creation}

The photometric redshift calibration, photometric catalogue creation and the photometric redshift estimation are presented in Brimioulle et al. (2013). We only give a brief overview here.

The estimation of meaningful colours from aperture fluxes requires same or at least similar shape of the point spread function (PSF) in the different filters of one pointing. Therefore in the first step we adjust the PSF by convolving all images of one pointing/filter with a fixed Gaussian kernel, degrading the PSF to the value of the worst band (in general $u$ ). We select the appropriate kernel in an iterative process, so the observational stellar colours no longer depend on the diameter of the circular aperture they are measured in. We then run SExtractor ${ }^{7}$ (see Bertin \& Arnouts 1996) in dual-image-mode, selecting the unconvolved $i$-band as detection band and extracting the photometric information from the convolved images. We extract all objects which are at least $2 \sigma$ above the background on at least four contiguous pixels.

Unfortunately the original magnitude zeropoint determination by the Elixir pipeline proved to be inaccurate. The colours of stars and galaxies can vary from field to field due to galactic extinction and because of remaining zero-point calibration errors. Since the CFHTLS-Wide fields are selected to be off the galactic plane, the extinction is rather small and does not change a lot over one square

\footnotetext{
2 http://cfhtlens.org

3 http://www4.cadc-ccda.hia-iha.nrc-cnrc.gc.ca/cadc

4 http://www.astromatic.net/software/scamp

5 http://www.astromatic.net/software/swarp

6 http://marvinweb.astro.uni- bonn.de/data

products/THELIWWW/automask.html

$7 \mathrm{http} / / / \mathrm{www}$.astromatic.net/software/sextractor
} 
degree tiles: the maximum and minimum extinction in all Wide fields is 0.03 and 0.14 , respectively, and the difference between maximum and minimum extinction value per square degree can be up to 0.03 for high extinction fields and 0.01 for fields with low extinction values. We account for one zero-point and extinction correction value per square degree field by shifting the observed stellar colours to those predicted from the Pickles stellar library (Pickles 1998) for the given photometric system. In this way we do not only correct for the inaccurate magnitude zeropoints, but do also correct for galactic extinction and field-to-field zeropoint variations.

\section{4 redMaPPer}

redMaPPer (Rykoff et al. 2014) is a red-sequence photometric cluster finding procedure that builds an empirical model for the redsequence colour-magnitude relation of early type cluster galaxies. It is built around the optimized richness estimator developed in Rozo et al. (2009) and Rykoff et al. (2012). redMaPPer detects clusters as overdensities of red galaxies, and measures the probability that each red galaxy is a member of a cluster according to a matched filter approach that models the galaxy distribution as the sum of a cluster and background component. The main design criterion for redMaPPer is to provide a galaxy count based mass proxy with as little intrinsic scatter as possible. To this end, member galaxies are selected at luminosities $L>0.2 L_{\star}$, based on their match to the red-sequence model, and with an optimal spatial filter scale (see Rykoff et al. 2016).

The redMaPPer richness of clusters is the sum of the membership probabilities of all galaxies. The aperture used as a cluster radius to estimate the cluster richness is self-consistently computed with the cluster richness, ensuring that richer clusters have larger cluster radii. This radius is selected to minimize the scatter of richness estimates at a given mass. The cluster richness estimated by redMaPPer has been shown to be strongly correlated with cluster mass by comparing the richness to well-known mass proxies such as X-ray gas mass and Sunyaev-Zel'dovich (SZ) decrements. The main (v5.2) redMaPPer algorithm was presented in Rykoff et al. (2014), to which the reader is referred for more details.

Especially at higher cluster redshift, the shallow SDSS photometry only allows for a relatively uncertain estimate of richness due to incompleteness at a magnitude corresponding to galaxies fainter than the redMaPPer limit of $0.2 L_{\star}$. The acquired follow-up CFHT photometry is significantly deeper, and therefore allows for improved estimates of $\lambda$ for the observed CODEX lensing sample. This, however, requires an independent calibration of the red sequence in the used set of filters $g$.MP9401, $r$.MP9601, $i$.MP9702 and $z$.MP9801. In section 4, we calibrate the richness-mass relation based on these improved CFHT richness estimates, and use the observed SDSS richnesses only to determine the shape of the sampling function, as is described in subsection 4.3 .

Due to incomplete observations in $g$ and $z$ for some of the clusters in our sample, we perform this in three separate variants, namely based on griz, gri and riz photometry. In the case of CODEX35646, where no $i . \mathrm{MP0702}$ band data is available, we generate artificial magnitudes by adding the $i$.MP9702-colour of a red galaxy template at the cluster redshift to the available $i$.MP9701-magnitude of all galaxies in the field.

For calibrating the red sequence, we use the spectroscopic cluster redshifts (see Table $\mathrm{C} 1$ and Table C2 ), where available. To account for masking to correct galaxy counts for undetected members, we convert the polygon masks applied to the CFHT object

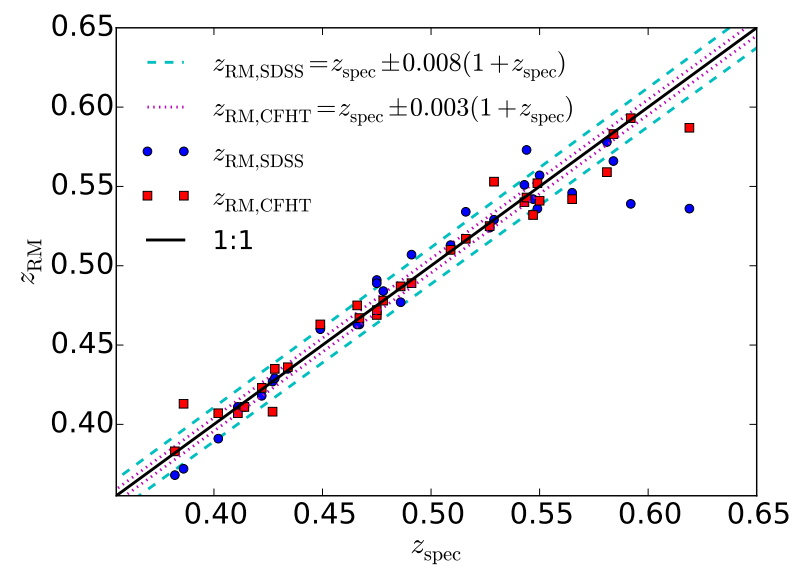

Figure 1. Spectroscopic redshifts versus CFHT/SDSS photometric cluster redshift estimates by redMaPPer for all spectroscopically covered clusters. Through a comparison with the spectroscopic redshifts of clusters, we measure photometric redshift precision of $\sigma_{\Delta_{z_{\mathrm{RM}} \text { SDSS }}} /\left(1+z_{\text {spec }}\right)=0.008$ and $\sigma_{\Delta_{z_{\mathrm{RM}, \mathrm{CFHT}}} /\left(1+z_{\text {spec }}\right)}=0.003$.

catalogues to a HEALPIX mask (Górski et al. 2005) with $N_{\text {side }}=$ 4096.

Using the spectroscopic redshifts obtained for this sample we can verify redMaPPer redshift determination. Fig. 1 shows spectroscopic redshift of the cluster BCGs versus the redMaPPer photometric redshift estimate $z_{\mathrm{RM}}$. Through this comparison the photometric redshift precision for both samples of SDSS and CFHT are found to correspond to $\sigma_{\Delta_{z_{\mathrm{RM}, S D S S}}} /\left(1+z_{\mathrm{spec}}\right)=$ 0.008 and $\sigma_{\Delta_{z_{\mathrm{RM}, \mathrm{CFHT}}}} /\left(1+z_{\mathrm{spec}}\right)=0.003$. While the redMaPPer photometric redshift precision of the SDSS-DR8 catalog is $\sigma_{z_{\text {SDSS,DR8 }} /\left(1+z_{\text {spec }}\right)}=0.006$, as estimated by Rykoff et al. (2014).

\subsection{Shape measurement}

We use the LENSFIT algorithm (see Miller et al. 2013) to measure galaxy shapes. We chose the $i$-band images for shape extraction as this band has usually smaller FWHM and lower atmospheric differential diffraction than the bluer bands.

The extracted quantities are the measured ellipticity components $e_{1}$ and $e_{2}$ and the weight taking into account shape measurement errors and the expected intrinsic shape distribution as defined in Miller et al. (2013). In order to sort out failed measurements and stellar contamination of our background sample we only consider background objects with a lensfit weight greater than 0 and a lensfit fitclass equal to 0 .

For our sample S-I we make use of the latest 'self-calibrating' version of the lensfit shape measurement (see Fenech Conti et al. 2017). Here we only highlight a few important facts about the selfcalibration, for a detailed description we refer the reader to its first application in the Kilo-Degree Survey (KiDS, see Fenech Conti et al. 2017; Hildebrandt et al. 2017). The main motivation for the self-calibration is given by the noise bias problem plaguing shape measurements techniques (see e.g. Melchior \& Viola 2012; Refregier et al. 2012; Miller et al. 2013; Fenech Conti et al. 2017; Kannawadi et al. 2019). However, the self-calibration is not perfect as it is shown to contain a residual calibration of the order of 2 per cent. Fenech Conti et al. (2017) discussed how to further reduce this 
with help of image simulations to the sub-per cent level as required for cosmic shear studies as presented by Hildebrandt et al. (2017), but given the residual statistical uncertainties in our cluster lensing studies, we discard this step and use the self-calibrated shapes directly. We estimate the uncertainty associated with this step to be around 3-5 percent of the actual shear value.

\subsection{Source selection and redshift estimation}

The observable in a weak lensing analysis is the mean tangential component of reduced gravitational shear $g_{\mathrm{t}}$ (see equation 5) of an ensemble of sources. At a given projected radius $r$ from the centre of the lens, it is related to the physical surface mass density profile of the latter, $\Sigma(r)$, by

$$
g_{\mathrm{t}}(r)=\frac{\Delta \Sigma(r) / \Sigma_{\text {crit }}}{1-\Sigma(r) / \Sigma_{\text {crit }}}+\text { Noise }
$$

where we have defined $\Delta \Sigma(r)=\left\langle\Sigma\left(r^{\prime}\right)\right\rangle_{r^{\prime}<r}-\Sigma(r)$. In the limit where $\Sigma \ll \Sigma_{\text {crit }}, g_{t}$ is equal to the tangential gravitational shear $\gamma_{t}$,

$$
g_{t}(r) \approx \gamma_{t}(r)=\Delta \Sigma(r) / \Sigma_{\text {crit }} .
$$

The critical surface mass density,

$$
\Sigma_{\text {crit }}=\frac{c^{2}}{4 \pi G D_{\mathrm{d}}} \frac{D_{\mathrm{s}}}{D_{\mathrm{ds}}},
$$

is a function of the angular diameter distances between the observer and lens $D_{\mathrm{d}}$, observer and source $D_{\mathrm{s}}$, and lens and source $D_{\mathrm{ds}}$. The ratio of the latter two is denoted in the following as the shorthand

$$
\beta=\frac{D_{\mathrm{ds}}}{D_{\mathrm{s}}}
$$

This is the part of equation 3 that depends on source redshifts, illustrating that the latter need to be known for converting lensing observables to physical surface densities.

Based on five-band photometry, redshifts of individual galaxies cannot be estimated unambiguously. However, since the lensing signal of each cluster is measured as the mean $\left\langle g_{t}\right\rangle$ over a large number of galaxies, for an unbiased interpretation of the signal it is sufficient to know the overall redshift distributions of the lensing-weighted source sample only. Here, we do this by defining subregions of the CFHT color-magnitude space with a decision tree algorithm. Each source galaxy can then be assigned to one of these subregions. A reference sample of galaxies with measurements in the same and additional photometric bands can be assigned to the same subregions. The redshift distribution of galaxies in each subregion can be estimated as the histogram of the high-quality photometric redshifts for the reference sample of galaxies assigned to the same subregion. The redshift distribution of the whole sample is a linear combination of the redshift distributions of the contributing subregions.

To this end, we follow the same algorithm as in Cibirka et al. (2016), described in more detail in Gruen \& Brimioulle (2016). In a nutshell, we divide five-band colour-magnitude space into boxes (hyper-rectangle subregions) and estimate the redshift distribution in each box from a reference catalog of 9-band optical+near-Infrared photo- $z$.

The reference catalogue of high-quality photo- $z$ is based on a magnitude-limited galaxy sample with 9band (u.MP9301, g.MP9401, r.MP9601, i.MP9701, i.MP9702, z.MP9801, J.WC8101, H.WC8201, Ks.WC8302)-photometry from the four pointings of the CFHTLS Deep and WIRCam Deep
(Bielby et al. 2012) Surveys. The outlier rate of these redshift estimates is $\eta=2.1$ per cent, with a photo- $z$ scatter $\sigma_{\Delta z /(1+z)}=0.026$ for $i<24$ (see Gruen \& Brimioulle 2016, their Fig. 4). We emphasize that the photometric catalogues in this work and the reference catalogue in Gruen \& Brimioulle (2016) have been created in the exact same way. In order to reduce contamination and enhance signalto-noise-ratio we apply several cuts during the construction of the colour-magnitude decision tree, as in Cibirka et al. (2016). This way, we remove parts of colour-magnitude space in which contamination with galaxies at the cluster redshift is possible. In addition, we identify and remove parts of color-magnitude space in which our 9-band photometric redshifts disagree with the COSMOS2015 photo- $z$ of Laigle et al. (2016). We also use the latter catalog to identify systematic uncertainties due to potential remaining biases in the high-quality photo- $z$ (see Appendix A2).

To perform the cuts described above, before construction of the decision tree we remove all galaxies from cluster and reference fields whose colour is in the range spanned by galaxies in the reference catalogue best fitted by a red galaxy template in the redshift interval $z_{\mathrm{d}} \pm 0.04$.

After construction of the decision tree we remove

- all galaxies in colour-magnitude hyper-rectangles for which $\langle\beta\rangle$ from COSMOS2015 photometric redshifts are below 0.2.

- all galaxies in colour-magnitude hyper-rectangles populated with any galaxies in the reference catalogue for which the redshift estimate is within $z_{\mathrm{d}} \pm 0.06$. In particular we remove all galaxies with a cprob-estimate unequal 0 to prevent contamination of the source sample with cluster members. We estimate the precision of the resulting estimate might still be biased up to a level of 2 per cent.

- all galaxies in colour-magnitude hyper-rectangles where the ratio of $\langle\beta\rangle$-estimates from COSMOS2015 versus our 9-band photometric redshifts deviates by more than 10 per cent from the median ratio over all hyper-rectangles.

The final estimate of the redshift distribution of a colormagnitude box comes from the 9-band photometric redshifts. We estimate the $\beta$ of a source galaxy as the mean $\beta$ of galaxies in the same box which it falls into. We refer the reader to Appendix A2 for details on systematic errors in the redshift calibration.

\subsection{Tangential shear and $\Delta \Sigma$ profile}

For a cluster $C$ and any radial bin $R$, we use the weighted mean of tangential ellipticities measured for a set of source galaxies $i$,

$$
g_{\mathrm{t}}(C, R)=\sum_{i} w_{i} \epsilon_{\mathrm{t}, i}
$$

where $\epsilon_{\mathrm{t}, i}$ is the component of the measured shape of galaxy $i$ tangential to the cluster centre and the sum runs over all sources around $C$ in a radial bin $R$, with weights $w_{i}$ that are normalized to $1=\sum_{i} w_{i}$.

Equivalently, in the limit of equation 2, we can estimate

$$
\Delta \Sigma(C, R)=\sum_{i} W_{i} \Delta \Sigma_{i}=\sum_{i} W_{i} \epsilon_{\mathrm{t}, i} /\left\langle\Sigma_{\mathrm{crit}, i}^{-1}\right\rangle,
$$

with a different set of weights $W_{i}$, again with $1=\sum_{i} W_{i}$. The expectation value of $\Sigma_{\text {crit }}^{-1}$ is calculated from equation 3 with the value of $\beta$ estimated in subsection 2.6. The statistically optimally weighted mean (i.e., the one with the highest signal-to-noise ratio) is achieved by using weights equivalent to the $\Delta \Sigma$ estimator of Sheldon 
et al. (2004), namely

$$
\begin{gathered}
w_{i} \propto \frac{\beta_{i}}{\sigma_{\mathrm{intr}}^{2}+\sigma_{\mathrm{obs}}^{2}}, \\
W_{i} \propto \frac{\left\langle\Sigma_{\mathrm{crit}, i}^{-1}\right\rangle^{2}}{\sigma_{\mathrm{intr}}^{2}+\sigma_{\mathrm{obs}}^{2}} \propto \frac{\beta_{i}^{2}}{\sigma_{\mathrm{intr}}^{2}+\sigma_{\mathrm{obs}}^{2}},
\end{gathered}
$$

where $\beta_{i}$ is the estimate of a galaxy's $\beta$ as described above, $\sigma_{\text {intr }}^{2}$ is the intrinsic variance of an individual component of galaxy ellipticity, and $\sigma_{\mathrm{obs}}^{2}$ is the variance in an individual component of galaxy shape due to observational uncertainty, both variances obtained from lensfit.

Equation 5 with these weights $w$ yields what we will call, in the following, mean tangential shear, and equation 6 with $W$ what we will call mean $\Delta \Sigma$. The above definitions and normalization conditions lead to the relation

$$
\Delta \Sigma(C, R)=g_{t}(C, R) /\left\langle\Sigma_{\text {crit }}^{-1}\right\rangle,
$$

where

$$
\left\langle\Sigma_{\text {crit }}^{-1}\right\rangle=\sum_{i} w_{i} \Sigma_{\text {crit }, i}^{-1}
$$

Mean shear and mean $\Delta \Sigma$ are therefore identical, up to normalization by the $w$-weighted mean of $\Sigma_{\text {crit }, i}^{-1}$. We do not show individual shear profiles, as they are rather noisy, but stacked profiles of the same cluster sample, that we have used in this work, can be found in Cibirka et al. (2016).

\subsection{Surface density model}

The interpretation of the weak lensing signal in order to derive a mass estimate for the galaxy cluster requires modelling of the surface density profile $\Sigma$. $\Sigma$ is related to the tangential reduced gravitational shear $g_{t}$ (equation 1) through the critical surface mass density (equation 3).

In our analysis we assume the galaxy cluster mass profile to follow a universal density profile, also known as NFW profile (see Navarro et al. 1996, 1997), which is described by

$$
\rho(r)=\frac{\delta_{\mathrm{c}} \rho_{\mathrm{c}}(z)}{\left(r / r_{\mathrm{s}}\right)\left(1+r / r_{\mathrm{s}}\right)^{2}},
$$

where $\rho_{\mathrm{c}}=\frac{3 H(z)^{2}}{8 \pi G}$ represents the critical density of the Universe at redshift $z, r_{s}$ refers to the scale radius where the logarithmic profile slope changes from -1 to -3 , and $\delta_{c}$ describes the characteristic over-density of the halo

$$
\delta_{\mathrm{c}}=\frac{200}{3} \frac{c^{3}}{\ln (1+\mathrm{c})-\mathrm{c} /(1+\mathrm{c})} .
$$

The characteristic over-density $\delta_{c}$ itself is a function of the so-called concentration parameter $c=r_{200} / r_{s}$.

For the explicit parametrizations for NFW shear components $\gamma_{\mathrm{t}}, g_{\mathrm{t}}$ and density contrast $\Delta \Sigma$ we refer to equations $11-16$ of Wright $\&$ Brainerd (2000). Note that the measured mean $\Delta \Sigma$ of equation 6 is equal to the true $\Delta \Sigma$ only in the weak shear limit, $\kappa \ll 1$, where $\kappa \equiv \Sigma(r) / \Sigma_{\text {crit }}$ denotes convergence, i.e. the dimensionless surfacemass density (cf. equation 1). To compensate the effect of reduced shear, we boost our model by $(1-\kappa)^{-1}$ when comparing it to the data.

In order to evaluate the weak lensing signal, we calculate the average of $\Delta \Sigma$ in logarithmically equidistantly binned annuli, both for the observational data and the analytic NFW profile that we use as a model. The radial range around the gravitational lens has to be chosen to minimize systematic effects but maximize our statistical power. Removing too much information on small scales results in loss of the region with the highest $\mathrm{S} / \mathrm{N}$. However, it is those small scales which are affected the most by off-centring. This subject will be investigated in further detail in Appendix A3 by examining simulated galaxy cluster halo profiles. As a trade-off, we decide to discard all background sources closer to the cluster centre than 500 $h^{-1} \mathrm{kpc}$, reducing a possible mass bias from off-centring to a minimum. On the other side large scales come with two effects. Firstly, the integrated NFW mass diverges for infinite scales, i.e. at a certain point the integrated analytic mass will exceed the physical cluster mass and thus bias low. On the other hand large scales start to be affected by higher-order effects as e.g. 2-halo-term, enhancing the observational mass profile, counter-acting at least partially the first effect. However, since these effects are not trivial to model, in our case the safer option is to discard those regions where these complicating effects start increasing, selecting as an outer analysis radius cut a distance of $2500 \mathrm{~h}^{-1} \mathrm{kpc}$. In a nutshell, we logarithmically bin our data in 12 radial annuli within 500 and $2500 \mathrm{~h}^{-1} \mathrm{kpc}$. Remaining biases by off-centring, large scale effects and other differences between our assumed NFW profile and the actual profile of galaxy clusters will be determined by calibration on recovered masses from simulated cluster halo profiles from Becker \& Kravtsov (2011) in Appendix A3 as mentioned before and be taken into account. Given this choice of scales, we fit mass only, fixing the concentration parameter by the concentration-mass relation of Dutton \& Macciò (2014) to

$$
\log _{10} \mathrm{c}=a+b \log _{10}\left(M /\left[10^{12} h^{-1} \mathrm{M}_{\odot}\right]\right),
$$

with

$$
b=-0.101+0.026 z
$$

and

$$
a=0.520+(0.905-0.520) \exp \left(-0.617 z^{1.21}\right)
$$

\subsection{Covariance matrix}

The measured profile $\Delta \Sigma_{\text {obs }}$ of any cluster of true mass $M$ deviates from the mean profile $\Delta \Sigma(M)$ of clusters of the same mass and redshift. In some annulus $i$, we can write

$$
\Delta \Sigma_{\mathrm{obs}, i}=\Delta \Sigma_{i}(M)+\delta_{i} .
$$

The covariance matrix element $C_{i j}$ required when determining a likelihood of $\Delta \Sigma_{\text {obs }}$ as a function of mass is the expectation value

$$
C_{i j}=\left\langle\delta_{i} \delta_{j}\right\rangle,
$$

which contains several components:

(i) shape noise, i.e. the scatter in measured mean shear due to intrinsic shapes and measurement uncertainty of shapes of background galaxies,

(ii) uncorrelated large-scale structure, i.e. statistical fluctuations of the matter density along the line of sight to the cluster, influencing the light path from the ensemble of background galaxies to the observer,

(iii) intrinsic variations of cluster profiles that would be present even under idealized conditions of infinite background source density and perfectly homogeneous lines of sight. 
All these components can be described as independent contributions to the covariance matrix, i.e.

$$
C_{i j}(M)=\left\langle\delta_{i} \delta_{j}\right\rangle=C_{i j}^{\mathrm{shape}}+C_{i j}^{\mathrm{LSS}}+C_{i j}^{\mathrm{intr}}(M) .
$$

We have made the dependence of the intrinsic variations of the cluster profile on mass $M$ explicit. The following sections describe these terms in turn. Since the overlap of annuli of pairs of different clusters in our sample is minimal, we assume that there is no crosscorrelation of shears measured around different clusters.

\subsubsection{Shape noise}

The lensfit algorithm provides the sum of intrinsic and measurement related variance of the ellipticity of each source $i, \sigma_{g, i}^{2}=\sigma_{\text {intr }}^{2}+\sigma_{\mathrm{obs}}^{2}$.

Using this to get the shape noise related variance in $\Delta \Sigma_{i}$,

$$
\sigma_{\Delta \Sigma, i}^{2}=\left(\frac{\sigma_{g, i}}{\left\langle\Sigma_{\text {crit }, i}^{-1}\right\rangle}\right)^{2} \propto W_{i}^{-1}
$$

the mean $\Delta \Sigma$ with the weights $W_{i}$ of equation 8 has a variance

$$
C_{i i}^{\text {shape }}=\frac{1}{\sum_{i} \sigma_{\Delta \Sigma, i}^{-2}} \propto \frac{1}{\sum_{i} W_{i}} .
$$

Due to the negligible correlation of shape noise between different galaxies, off-diagonal components are set to zero.

\subsubsection{Uncorrelated large-scale structure}

Random structures along the line of sight towards the source galaxies used for measuring the cluster shear profiles cause an additional shear signal of their own. The latter is zero on average, but has a variance (and co-variance between different annuli) that is an integral over the convergence power spectrum and therefore depends both on the matter power spectrum and the weighted distribution of source redshifts. We analytically account for this contribution to the covariance matrix as (e.g. Schneider et al. 1998; Hoekstra 2003; Umetsu et al. 2011; Gruen et al. 2015)

$$
C_{i j}^{\mathrm{LSS}}=\int \frac{\ell \mathrm{d} \ell}{2 \pi} P_{\kappa}(\ell) \hat{J}_{0}\left(\ell \theta_{i}\right) \hat{J}_{0}\left(\ell \theta_{j}\right) .
$$

Here, $\hat{J}_{0}\left(\ell \theta_{i}\right)$ is the area-weighted average of the Bessel function of the first kind $J_{0}$ over annulus $i$. The convergence power spectrum $P_{\kappa}$ is obtained from the matter power spectrum by the Limber (1954) approximation as

$$
\begin{array}{r}
P_{K}(\ell)=\frac{9 H_{0}^{2} \Omega_{m}^{2}}{4 c^{2}} \quad \int_{0}^{\chi_{\max }} \mathrm{d} \chi a^{-2}(\chi) P_{\mathrm{nl}}(\ell / \chi, \chi) \\
\int_{\chi}^{\chi_{\max }} \mathrm{d} \chi_{s} p\left(\chi_{s}\right)\left(\frac{\chi_{s}-\chi}{\chi_{s}}\right)^{2} .
\end{array}
$$

Here $\chi$ denotes comoving distance to a given redshift, and $p\left(\chi_{s}\right)$ is the PDF of comoving distance to sources in the lensing sample, defined as the sum of each individual source PDF (subsection 2.6), weighted by the $w$ of equation 7. For the non-linear matter power spectrum $P_{\mathrm{nl}}$ we use the model of Smith et al. (2003) with the Eisenstein \& Hu (1998) transfer function including baryonic effects. Note that since the source sample, weighting, and angular size of annuli is different for each cluster, we calculate a different $C^{\mathrm{LSS}}$ for each one of them.

\subsubsection{Intrinsic variations of cluster profiles}

Even under perfect observing conditions without shape noise, and in the hypothetical case of a line of sight undisturbed by inhomogeneities along the line of sight, the shear profiles of a sample of clusters of identical mass would still vary around their mean. The reason for this are intrinsic variations in cluster profiles, halo ellipticity and orientation, and subhaloes in their interior and correlated environment. We describe these variations using the semi-analytic model of Gruen et al. (2015), which proposes templates for each of these components and determines their amplitudes to match the actual variations of true cluster profiles at fixed mass seen in simulations (Becker \& Kravtsov 2011). We write

$$
C_{i j}^{\mathrm{intr}}(M)=C_{i j}^{\mathrm{conc}}(M)+C_{i j}^{\mathrm{ell}}(M)+C_{i j}^{\mathrm{corr}}(M)+C_{i j}^{\mathrm{off}}(M),
$$

where we assume the best-fit re-scaled model of Gruen et al. (2015) for the contributions from halo concentration variation $C_{i j}^{\text {conc }}$, halo ellipticity and orientation $C_{i j}^{\text {ell }}$ and correlated secondary haloes $C_{i j}^{\text {corr }}$. For the purpose of this work, the templates in Gruen et al. (2015) are resampled from convergence to shear measurement and re-scaled to the $\Delta \Sigma$ units of our measurement with the weighted mean $\Sigma_{\text {crit }}$ of the source sample. The final component, $C_{i j}^{\text {off }}$ is added to account for variations in off-centring width of haloes. It is calculated as the covariance of shear profiles of haloes of fixed mass, with miscentring offsets drawn according to the prescription of Rykoff et al. (2016). We note that each of these components depends on halo mass, halo redshift, and angular binning scheme. We therefore calculate a different $C_{i j}^{\text {intr }}(M)$ for each cluster in our sample. The code producing these covariance matrices is available at https://github.com/danielgruen/ccv.

\subsection{Mass likelihood}

The lensing likelihood for an individual cluster is proportional to the probability of observing the present mean $\Delta \Sigma$ given a true cluster mass $M=M_{200 c}$. Assuming multivariate Gaussian errors in the observed signal, it can be written as

$$
p(\Delta \Sigma \mid M) \propto \frac{1}{\sqrt{\operatorname{det} C(M)}} \times \exp \left(-\frac{1}{2} \boldsymbol{E}(M)^{\mathrm{T}} C^{-1}(M) \boldsymbol{E}(M)\right),
$$

where $\boldsymbol{E}$ is the vector of residuals between data and model evaluated at mass $M$,

$$
E_{i}(M)=\Delta \Sigma_{i}^{\mathrm{obs}}-\Delta \Sigma_{i}^{\mathrm{model}}(M)
$$

and $C$ is the covariance matrix (cf. equation 16). The mass dependence of the covariance, due entirely to $C_{\mathrm{intr}}$, causes a complication relative to a simple minimum- $\chi^{2}$ analysis: the normalization of the Gaussian PDF depends on mass that needs to be accounted for by the $\operatorname{det}^{-1 / 2} C(M)$ term in equation 22 . If the covariance is modelled perfectly, including the mass dependence, the above is the correct likelihood (see e.g. Kodwani et al. 2019). If, however, the mass dependence of the covariance is modeled with some statistical or systematic uncertainty, the $\operatorname{det}^{-1 / 2} C(M)$ term can cause a bias in the best-fit masses.

For this reason, we use a two-step scheme:

(i) determine the best-fit mass using a covariance that consists of shape noise and LSS contributions only, i.e. has no mass dependence

(ii) evaluate $C^{\text {intr }}$ at the best fit mass of step (i), add this to the covariance without mass dependence and repeat the likelihood analysis with this updated, full, yet mass-independent covariance. 
Table 1. Main weak lensing sample $\left(\lambda_{\mathrm{RM}, \mathrm{SDSS}}>60\right.$ and $\left.z \geqslant 0.35\right)$ of 25 clusters

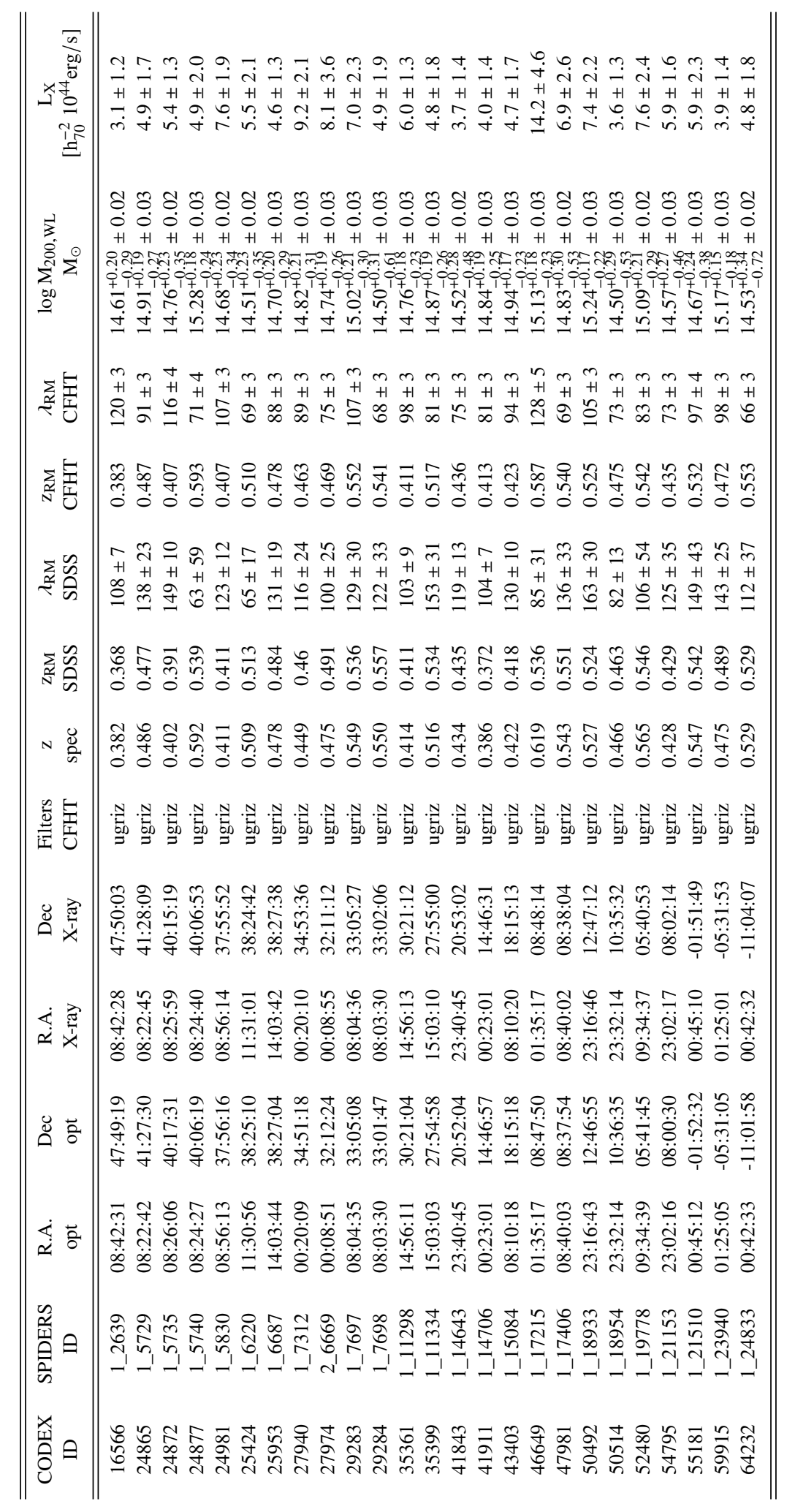




\section{HIERARCHICAL BAYESIAN MODEL}

Below we describe the hierarchical Bayesian model, which we use to determine the posterior distribution of the parameters of interest. The following section follows a similar framework as in Nagarajan et al. (2018) and Mulroy et al. (2019), except, instead of one selection function, we introduce two separate selection functions, the CODEX selection function and the sampling function, for our lensing subsample.

The true underlying halo mass of the cluster $i$ in log-space $\mu_{i}=\ln \left(M_{i}\right)$ is related to all other observables through a scaling model $P\left(s_{i}, \mu_{i} \mid \theta\right)$, where $s_{i}=\ln \left(S_{i}\right)$ is the vector of true quantities in $\log$-space and $\boldsymbol{\theta}$ represents a vector of parameters of interest. At given redshift, the joint probability distribution that there exist a cluster of mass $\mu_{i}$ can be written as

$$
P\left(s_{i}, \mu_{i} \mid \boldsymbol{\theta}, z_{i}\right)=P\left(s_{i} \mid \mu_{i}, \boldsymbol{\theta}\right) P\left(\mu_{i} \mid z_{i}\right) P(z),
$$

where we model the conditional distribution for the mass at given redshift $z_{i}, P\left(\mu_{i} \mid z_{i}\right)$, as the halo mass function (HMF) $\frac{d n}{d \ln m}\left(\mu_{i} \mid z_{i}\right)$ and $P(z)$ is the comoving differential volume element $d V / d z(z)$. In practice, $P\left(\mu_{i} \mid z_{i}\right)$ is evaluated as a Tinker et al. (2008) mass function using fixed $\Lambda \mathrm{CDM}$ cosmology, where $\Omega_{m}=0.27, \Omega_{\Lambda}=0.73$, $\Omega_{b}=0.049, H_{0}=70 \mathrm{~km} \mathrm{~s}^{-1} \mathrm{Mpc}^{-1}, \sigma_{8}=0.82, n_{s}=0.962$, for a density contrast of $200 \times \rho_{c}$.

The underlying true values of the observables in log-space $s_{i}$ are assumed to come from a multivariate Gaussian distribution:

$$
P\left(s_{i} \mid \mu_{i}, \boldsymbol{\theta}\right) \propto \operatorname{det}\left(\boldsymbol{\Sigma}_{i}^{-1 / 2}\right) \exp \left[-\frac{1}{2}\left(s_{i}-\left\langle s_{i}\right\rangle\right)^{T} \boldsymbol{\Sigma}_{i}^{-1}\left(s_{i}-\left\langle s_{i}\right\rangle\right)\right],
$$

where the mean of the probability distribution of observables is modelled as a linear function in log-space $\left\langle\boldsymbol{s}_{i}\right\rangle=\alpha \mu_{i}+\boldsymbol{\beta}$. The model parameters are defined as $\boldsymbol{\theta}=\left\{\boldsymbol{\alpha}, \boldsymbol{\beta}, \boldsymbol{\Sigma}_{i}\right\}$, where $\boldsymbol{\alpha}$ is the vector of slopes, $\boldsymbol{\beta}$ is vector of intercepts and $\boldsymbol{\Sigma}$ is the intrinsic covariance matrix of the cluster observables at fixed mass. The diagonal elements of the intrinsic covariance matrix, $\sigma_{\ln s_{i} \mid \mu}$, represent the intrinsic scatter for a cluster observable $s_{i}$ at fixed mass. The off-diagonal elements are the covariance terms between different cluster observables at fixed mass.

However, we cannot directly access cluster observables, but only have estimates through observations, which contain observational uncertainties. We denote the observed logarithmic quantities with tilde: $\tilde{s}_{i}, \tilde{\mu}_{i}, \tilde{z}_{i}$, and the vector of all observables as $\tilde{\mathbf{o}} \in\left\{\tilde{\boldsymbol{s}}_{i}, \tilde{\mu}_{i}, \tilde{z}_{i}\right\}$. To connect them to their respective underlying true observables $\mathbf{o} \in\left\{\mathbf{s}_{\mathbf{i}}, \mu_{i}, z_{i}\right\}$, we assume the full lensing likelihood from equation 22 for the mass, which we denote here $P\left(\tilde{\mu}_{i} \mid \mu_{i}\right)$, and, for other parameters, a multivariate Gaussian distribution $P\left(\tilde{\boldsymbol{s}}_{i}, \tilde{z}_{i} \mid \boldsymbol{s}_{i}, z_{i}\right)$, which acts as our measurement error model:

$$
P\left(\tilde{\boldsymbol{s}}_{i}, \tilde{z}_{i} \mid \boldsymbol{s}_{i}, z_{i}\right) \propto \operatorname{det}\left(\tilde{\boldsymbol{\Sigma}}_{i}^{-1 / 2}\right) \exp \left[-\frac{1}{2}\left(\tilde{\boldsymbol{s}}_{i}-\boldsymbol{s}_{i}\right)^{T} \tilde{\boldsymbol{\Sigma}}_{i}^{-1}\left(\tilde{\boldsymbol{s}}_{i}-\boldsymbol{s}_{i}\right)\right] .
$$

The diagonal elements of the covariance matrix in equation 26 represent the relative statistical errors in the observables for cluster $i$ and the off-diagonal elements the covariance between the relative errors of different observables. In practice, instead of using the evaluated richness measurement errors from the redMaPPer algorithm, we assume a Poisson noise model, described further in equations 36 and 37 . For simplicity, for a single cluster, we expect independent measurement errors between different observables.

For the total population, the probability of measuring the observed cluster property $\tilde{\boldsymbol{s}}_{i}$ for a single cluster $i$ at fixed observed mass $\tilde{\mu}_{i}$ and observed redshift $\tilde{z}_{i}$, can be expressed as

$$
\begin{aligned}
P\left(\tilde{\boldsymbol{s}}_{i}, \tilde{\mu}_{i}, \tilde{z}_{i} \mid \boldsymbol{\theta}\right)= & \int d \boldsymbol{s}_{i} \int d \mu_{i} \int d z_{i} P\left(\tilde{\boldsymbol{s}}_{i}, \tilde{z}_{i} \mid \boldsymbol{s}_{i}, z_{i}\right) \\
& \cdot P\left(\tilde{\mu}_{i} \mid \mu_{i}\right) P\left(\boldsymbol{s}_{i} \mid \mu_{i}, \boldsymbol{\theta}\right) P\left(\mu_{i} \mid z_{i}\right) P(z) .
\end{aligned}
$$

Note that in equation 27 , we have to marginalize over all the unobserved cluster properties, i.e., underlying halo mass, true observables and true redshift.

In reality, one cannot directly observe the full population of clusters, but a subsample of it based on some easily observable cluster property, such as luminosity or richness of the cluster. In order to rectify the bias coming from the observed censored population, one has to include the selection process in the model. If the selection is done several times with different observables, e.g., taking a subsample from a sample that represents the population, one should introduce all different selection processes into the modelling.

In order to introduce a selection effect into the Bayesian modelling, we define a boolean variable for the selection $I$, which we will use as a conditional variable to specify whether a cluster is detected or not. Let's first consider a single selection variable $\tilde{\lambda}$. Assume we have made a cut at $\tilde{\lambda}$, and we observe all the clusters above this limit. Then $P(I=1 \mid \tilde{\lambda} \geqslant$ cut $)=1$ for all observed clusters, and $P(I=0 \mid \tilde{\lambda}<$ cut $)=0$, for all unobserved clusters.

However, if we don't detect all the clusters above the cut, just a subsample of clusters, but know how many clusters we miss, we can calculate the fraction of clusters from the subsample that belong to the sample at certain richness $f\left(\tilde{\lambda}_{i, \text { sub }}\right)=\tilde{N}_{\text {sub }} / \tilde{N}_{\text {sample }}\left(\tilde{\lambda}_{i, \mathrm{sub}}\right)$, and treat this fraction as our subsample detection probability, for which $P\left(I=1 \mid \tilde{\lambda}_{i \text {, sub }}\right)=f\left(\tilde{\lambda}_{i \text {, sub }}\right) \leqslant 1$. We note that $f$ returns to the heaviside step function, if we observe all the clusters above the cut $\tilde{\lambda}$. Below, we generalize the selection probability $P\left(I \mid \tilde{\mathbf{o}}_{i}, \boldsymbol{\theta}\right)$ by considering any selection function to depend on multiple selection variables $\tilde{\mathbf{o}}_{i}$, and the vector of parameters of interest $\boldsymbol{\theta}$.

Using the Bayes' theorem, the probability of measuring the observed cluster properties $\tilde{\mathbf{o}}_{i}$, given fixed vector of parameters $\boldsymbol{\theta}$ and that the cluster passed the selection is

$$
P\left(\tilde{\mathbf{o}}_{i} \mid I, \boldsymbol{\theta}\right)=\frac{P\left(I \mid \tilde{\mathbf{o}}_{i}, \boldsymbol{\theta}\right) P\left(\tilde{\mathbf{o}}_{i} \mid \boldsymbol{\theta}\right)}{P(I \mid \boldsymbol{\theta})},
$$

where $P\left(I \mid \tilde{\mathbf{o}}_{i}, \boldsymbol{\theta}\right)$ quantifies the probability of detecting a single cluster, and $P(I \mid \boldsymbol{\theta})$, is the overall probability for all the clusters to be selected, which can be evaluated by marginalizing over the observed cluster properties from the numerator in equation 28 :

$$
P(I \mid \boldsymbol{\theta})=\int d \tilde{\mathbf{o}}_{i} P\left(I \mid \tilde{\mathbf{o}}_{i}, \boldsymbol{\theta}\right) P\left(\tilde{\mathbf{o}}_{i} \mid \boldsymbol{\theta}\right) .
$$

In the case where the selection depends on both observed and true quantities, equation 28 becomes, according to Bayes theorem:

$$
P\left(\tilde{\mathbf{o}}_{i}, \mid I_{\mathrm{tot}}, \boldsymbol{\theta}\right)=P\left(\tilde{\mathbf{o}}_{i}, \mid I_{\mathrm{obs}}, I_{\mathrm{true}}, \boldsymbol{\theta}\right)=\frac{P\left(I_{\mathrm{obs}} \mid \tilde{\mathbf{o}}_{i}, \boldsymbol{\theta}\right) P\left(I_{\mathrm{true}}, \tilde{\mathbf{o}}_{i} \mid \boldsymbol{\theta}\right)}{P\left(I_{\mathrm{obs}}, I_{\mathrm{true}} \mid \boldsymbol{\theta}\right)},
$$

where we have introduced a second selection parameter $I_{\text {true }}$, that denotes the selection based on true quantities. The first term is the same selection function $P\left(I \mid \tilde{\mathbf{o}}_{i}, \boldsymbol{\theta}\right)$ as in equation 28 , and the second term in the numerator can be expressed as

$$
\begin{array}{r}
P\left(I_{\text {true }}, \tilde{\mathbf{o}}_{i} \mid \boldsymbol{\theta}\right)=\int d \boldsymbol{s}_{i} \int d \mu_{i} P\left(I_{\text {true }} \mid \boldsymbol{s}_{i}, \mu_{i}\right) \\
\cdot P\left(\tilde{\boldsymbol{s}}_{i}, \tilde{\mu}_{i} \mid \boldsymbol{s}_{i}, \mu_{i}\right) P\left(\boldsymbol{s}_{i}, \mu_{i} \mid \boldsymbol{\theta}\right) .
\end{array}
$$

Equation 27 is assumed to work only if no censoring is involved, but equation 31 assumes that the observed set belongs to a larger population, and the selection $P\left(I_{\text {true }} \mid s_{i}, \mu_{i}\right)$ can be modelled 
with simulations, where the true observables are known. In section 4.2, we introduce the CODEX X-ray selection, $P\left(I_{X} \mid s_{i}, \mu_{i}\right)$, which is defined as a function of true observables.

The normalization of the likelihood function in equation 30 can also be expressed as an integral over all observables:

$$
P\left(I_{\mathrm{obs}}, I_{\text {true }} \mid \boldsymbol{\theta}\right)=\int d \tilde{\mathbf{o}}_{i} P\left(I_{\mathrm{obs}} \mid \tilde{\mathbf{o}}_{i}, \boldsymbol{\theta}\right) P\left(I_{\text {true }}, \tilde{\mathbf{o}}_{i} \mid \boldsymbol{\theta}\right) .
$$

Finally, the full likelihood function for the subsample, with the inclusion of the selection effects, becomes a product of the single cluster likelihood functions from equation 30 :

$$
\mathcal{L}\left(\tilde{\mathbf{o}}_{N} \mid \boldsymbol{\theta}\right)=\prod_{i=1}^{N} P\left(\tilde{\mathbf{o}}_{i} \mid I_{\mathrm{tot}}, \boldsymbol{\theta}\right),
$$

where subscript $\mathrm{N}$ denotes the full vector of observed measurements from all the clusters. The full posterior distribution, which describes the probability distribution of parameters of interest, given the observed mass, redshift and set of observables is then

$$
P\left(\boldsymbol{\theta} \mid \tilde{\boldsymbol{s}}_{N}, \tilde{\mu}_{N}, \tilde{z}_{N}\right) \propto \pi(\boldsymbol{\theta}) \mathcal{L}\left(\tilde{\boldsymbol{s}}_{N}, \tilde{\mu}_{N}, \tilde{z}_{N} \mid \boldsymbol{\theta}\right),
$$

where $\pi(\boldsymbol{\theta})$ describes the prior knowledge of the parameters.

\section{APPLICATION TO THE CODEX WEAK LENSING SAMPLE}

We apply the above described Bayesian method to the lensing sample S-I, and exclude eleven clusters: CODEX ID 53436 and 53495 as they are missing both CFHT richness and weak lensing information; 37098 as it is missing weak lensing information; 13390, 29811 and 56934 as they are missing CFHT richness information; CODEX ID 13062 (griz) and 35646 (griz) as we only employed our method to clusters measured with five filters (ugriz); CODEX ID 12451,18127 and 36818 as their CFHT richness are below the $10 \%$ CODEX survey completeness limit, which is further described in section 4.1 .

We aim to constrain both the intrinsic scatter in richness and the scaling relation parameters describing the richness-mass relation, see equation 35. For that we fit a model of richness-mass relation to CFHT richness estimates and weak lensing mass likelihood (see Table C1 for CFHT richness estimates). We don't fit for the SDSS richness-mass relation as the SDSS richness estimates have mean relative uncertainty of $\sim 20 \%$, in contrast to CFHT richness mean relative uncertainty of $\sim 4 \%$. However, since the lensing sample of 25 clusters, i.e., a subsample of the initial CODEX sample, is based purely on observability, such that not all clusters above the $\tilde{\lambda}_{\text {SDSS }}=60$ cut are observed, we use the fraction of SDSS richnesses $P\left(I=1 \mid \ln \tilde{\lambda}_{\text {SDSS }}\right)$ as our subsample selection function, and treat the SDSS richness in our likelihood function as one of the selection variables, which we will marginalize over. As for CFHT and SDSS richnesses, we assume both are coming from the similar log-normal richness distribution, i.e., $P(\ln \tilde{\lambda} \mid \ln \lambda)=\mathcal{N}\left(\ln \tilde{\lambda} ; \ln \lambda, \sigma_{\ln \lambda}\right)$, but with somewhat larger scatter for the SDSS richness, which is described below.

The relation between underlying true richness and true mass of the cluster is assumed to be a Gaussian distribution in logarithmic space, with the mean of this relation given by the logarithm of a power-law:

$$
\left\langle\ln \lambda_{i} \mid \mu_{i}\right\rangle=\alpha \mu_{i}+\beta,
$$

where we have defined $\mu_{i} \equiv \ln \left(M_{i} / M_{\text {piv }}\right)$ with pivot mass set to $M_{\text {piv }}=10^{14.81} M_{\odot}$, i.e., the median mass of the lensing subsample.
The model parameters of interest, $\alpha$ and $\beta$, describe the scaling relation slope and intercept, respectively. This parametrization follows Saro et al. (2015). We write the full scatter in $\tilde{\lambda}_{\text {SDSS }}$ as the sum in quadrature of a Poisson and an intrinsic variance terms. Thus, the total variance in observed SDSS richness at a fixed true mass $\mu_{i}$ can be written as (Capasso et al. 2019):

$$
\sigma_{\text {tot,SDSS }}^{2}\left(\ln \lambda_{i} \mid \mu_{i}\right)=\frac{\eta\left(z_{i}\right)}{\exp \left\langle\ln \lambda_{i} \mid \mu_{i}\right\rangle}+\sigma_{\ln \lambda \mid \mu, \text { intr }}^{2},
$$

where $\sigma_{\ln \lambda \mid \mu, \text { intr }}^{2}$ is the third free parameter of our model. As described in Capasso et al. (2019), a redshift dependent correction factor $\eta(z)$ is estimated for high redshift clusters to remedy the effect that the SDSS photometric data is not deep enough to correctly measure the richness after a certain magnitude limit is reached. As the CFHT photometric richnesses come from a sufficiently deep survey, we can set the survey depth correction factor to unity, so that the total variance in CFHT richness can be modelled as:

$$
\sigma_{\text {tot,CFHT }}^{2}\left(\ln \lambda_{i} \mid \mu_{i}\right)=\frac{1}{\exp \left\langle\ln \lambda_{i} \mid \mu_{i}\right\rangle}+\sigma_{\ln \lambda \mid \mu, \text { intr }}^{2} .
$$

We also test the Poisson term in terms of true richness, in contrast to mean richness, and the difference between these two error estimation methods are negligible.

For the observed mass estimation, we use the single cluster mass likelihood function $P(\tilde{\mu} \mid \mu)$, from equation 22 . We introduce a fourth scalar parameter, $l_{\text {sys }}$ with standard normal distributed prior, to draw how different the noiseless logarithmic lensing masses are from the true logarithmic masses due to imperfect calibration of lensing shapes, redshifts, and the cluster density profiles.

We assume that the observed spectroscopic redshift is close to the true redshift of the cluster, i.e., we model the term $P(\tilde{z} \mid z)$ as a delta function.

In the case the sample is only limited by observed richness $\tilde{\lambda}_{i}$, with the calibration of the richness-mass scaling relation based on weak lensing data, the probability distribution can be written according to equation 28. The initial CODEX sample contains both optical and X-ray selection. The X-ray selection requires the inclusion of the CODEX selection function, replacing equation 28 with equation 30 .

\subsection{Optical selection functions}

We consider two separate optical selection functions below that account for optical cleaning and incompleteness of the survey. We describe by $P\left(I_{\text {clean }} \mid \tilde{\lambda}, \tilde{z}\right)$ the optical cleaning applied to the catalog. In practice, this is a redshift dependent cut in observed richness used to minimize false X-ray sources while keeping as many true systems as possible. For the CODEX survey, this redshift cut is chosen by the $10 \%$ sensitivity limit. We adopt the $10 \%$ CODEX sensitivity limit

$$
P\left(I_{\text {clean }} \mid \tilde{\lambda}, \tilde{z}\right)= \begin{cases}1, & \text { if } \tilde{\lambda}>22\left(\frac{\tilde{z}}{0.15}\right)^{0.8} \\ 0, & \text { otherwise. }\end{cases}
$$

from Finoguenov et al. (2020) to CFHT richnesses to only account for clusters which have richness completeness over $10 \%$. This cut excludes three clusters from S-I (CODEX ID 12451, 18127, and 36818).

We also consider the 50\% SDSS richness completeness boundary:

$$
\ln \lambda_{50 \%}(z)=\ln \left(17.2+\exp \left(\frac{z}{0.32}\right)^{2}\right)
$$


i.e., clusters with SDSS richness above these limits have at least $50 \%$ completeness, respectively. We include the 50\% SDSS richness completion as an optical selection function

$$
P\left(I_{\mathrm{opt}} \mid \ln \lambda\right)=1-\frac{1}{2} \operatorname{erfc}\left(\frac{\ln \lambda-\ln \lambda_{50 \%}}{\sqrt{2} \sigma}\right)
$$

in the likelihood function with a scatter of $\sigma=0.2$, as described in Finoguenov et al. (2020). This term accounts for incompleteness due to limited photometric depth of the SDSS survey causing a fraction of clusters to go unobserved.

\subsection{X-ray selection function}

Details of the CODEX selection function are given in Finoguenov et al. (2020). The CODEX selection function $P\left(I_{X} \mid \mu, \mathrm{z}, v\right)$ provides an effective survey area at a given mass, redshift, and deviation from the mean richness at fixed mass $v \equiv \frac{\ln \lambda_{i}-\left\langle\ln \lambda \mid \mu_{i}\right\rangle}{\sigma_{\ln \lambda}^{\text {intr }}}$, which accounts for the covariance between scatter in richness and X-ray luminosity. The limits for $v$ is fixed between \pm 4 . In the modelling the CODEX selection function, the $L_{X}$-mass scaling relations are fixed to those by the XMM-XXL survey (Lieu et al. 2016; Giles, P. A. et al. 2016), but the richness-mass relation is not modelled explicitly in the selection function, only the covariance between richness and luminosity. For the selection function modelling, the covariance coefficient is fixed to $\rho_{\mathrm{L}_{X}-\lambda}=-0.3$, which is based on results from Farahi et al. (2019). In this work, the CODEX selection function is evaluated at fixed cosmology with $\Omega_{m}=0.27$. The formulation of selection function allows us to propagate these effects into the full selection function.

As the CODEX selection function depends on $v(\lambda,\langle\ln \lambda\rangle)$, and the mean richness in $v$ depends on scaling relation parameters, we can simplify the likelihood function by evaluating it in $v$-space instead of in $\lambda$-space. In $v$-space, equation 31 can be rewritten as

$$
\begin{aligned}
P\left(I_{X}, \ln \tilde{\lambda}, \tilde{\mu}, \tilde{z} \mid \boldsymbol{\theta}\right)= & \int d v \int d \mu \int d z P\left(I_{X} \mid \mu, v, z\right) P(\tilde{\mu} \mid \mu) P(\tilde{z} \mid z) \\
& \cdot P(\ln \tilde{\lambda} \mid v, \theta, \mu) P(v) P(\mu \mid z) P(z),
\end{aligned}
$$

which is the probability of observing a full sample with the inclusion of CODEX selection. However, we are dealing with a subsample, which gets selected with the sampling function, described below.

\subsection{Subsample selection function}

For evaluating the sampling function, based on SDSS richness, we use the initial CODEX sample (407 clusters, three light blue bins behind the three dark blue bins in Fig. 2) and its subsample ( 25 clusters, three dark blue bins in Fig. 2). We bin both the initial sample and the subsample, the lensing sample, into equal bin widths and evaluate the ratio of the height of the bins. We then fit a linear piecewise function between the mean of the bins, which becomes our sampling function that depends on observed SDSS richness, depicted by the orange curve in Fig. 2.

The sampling function has the following form:

$$
P\left(I_{\text {samp }} \mid \text { Kii } \tilde{\lambda}_{\text {SDSS }}\right)= \begin{cases}0 & \tilde{\lambda}<60 \\ \frac{1}{1000}(\tilde{\lambda}-60)+\frac{7}{1000} & 60 \leqslant \tilde{\lambda}<91 \\ \frac{33}{1000}(\tilde{\lambda}-91)+\frac{38}{1000} & 91 \leqslant \tilde{\lambda}<136 \\ \frac{186}{1000} & 136 \leqslant \tilde{\lambda} \leqslant 163,\end{cases}
$$

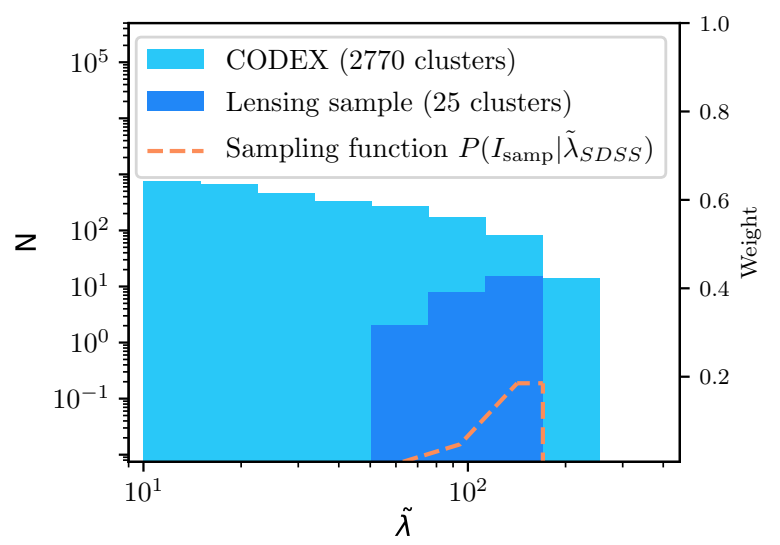

Figure 2. SDSS richness distributions of CODEX sample and lensing sample, from which the sampling function (weight as a function of observed richness) is derived.

As the clusters in the 407 cluster initial sample has cut at $\tilde{\lambda}_{\text {SDSS }} \geqslant 60$, the sampling function defines a null probability for clusters below this cut. Since the lensing sample, a subsample of the initial sample, is selected based only by observability, some of the clusters in the initial sample above the richness cut are unobserved, the sampling function differs from a typical heaviside step function.

The sampling function depends only on SDSS richness, which we can consider as an effective richness. We introduce an additional Gaussian distribution $P\left(\ln \tilde{\lambda}_{S D S S} \mid \ln \lambda\right)$ to account for the connection between SDSS richness and true richness and marginalize the likelihood function over the SDSS richness.

\subsection{Full data likelihood function}

Included for completeness is the full likelihood function in $v$-space that we use to constrain the parameters of interest $\theta=\left\{\alpha, \beta, \sigma_{\ln \lambda}^{\text {intr }}\right\}$ :

$$
\begin{aligned}
\mathcal{L}=\prod_{i=1}^{N} \phi\left(I_{X}, I_{\mathrm{samp}}, I_{\mathrm{opt}} \mid \theta\right)^{-1} & \int d v_{i} \int d \mu_{i} \int d \ln \tilde{\lambda}_{i, S D S S} \\
& \cdot P\left(I_{\mathrm{samp}} \mid \ln \tilde{\lambda}_{i, S D S S}\right) \\
& \cdot P\left(I_{X} \mid \mu_{i}, \tilde{z}_{i}, v_{i}\right) \\
& \cdot P\left(I_{\mathrm{opt}} \mid v_{i}, \mu_{i}, \theta\right) \\
& \cdot P\left(\ln \tilde{\lambda}_{i, S D S S} \mid v_{i}, \mu_{i}, \theta, \tilde{z}_{i}\right) \\
& \cdot P\left(\ln \tilde{\lambda}_{i, C F H T} \mid v_{i}, \mu_{i}, \theta\right) \\
& \cdot P\left(\tilde{\mu}_{i} \mid \mu_{i}\right) \\
& \cdot P\left(v_{i}\right) \\
& \cdot P\left(\mu_{i}, \tilde{z}_{i}\right)
\end{aligned}
$$

where the normalization of the likelihood is :

$$
\begin{aligned}
\phi\left(I_{X}, I_{\mathrm{samp}}, I_{\mathrm{opt}} \mid \theta\right)= & \int d v \int d \mu \int d \ln \tilde{\lambda}_{S D S S} \int d \tilde{z} \\
& \cdot P\left(I_{\mathrm{samp}} \mid \ln \tilde{\lambda}_{S D S S}\right) \\
& \cdot P\left(I_{X} \mid \mu, \tilde{z}, v\right) \\
& \cdot P\left(I_{\mathrm{opt}} \mid v, \mu, \theta\right) \\
& \cdot P\left(\ln \tilde{\lambda}_{S D S S} \mid v, \mu, \theta, \tilde{z}\right) \\
& \cdot P(v) \\
& \cdot P(\mu, \tilde{z}) .
\end{aligned}
$$

where $\tilde{\lambda} \equiv \tilde{\lambda}_{\text {SDSS }}$. 


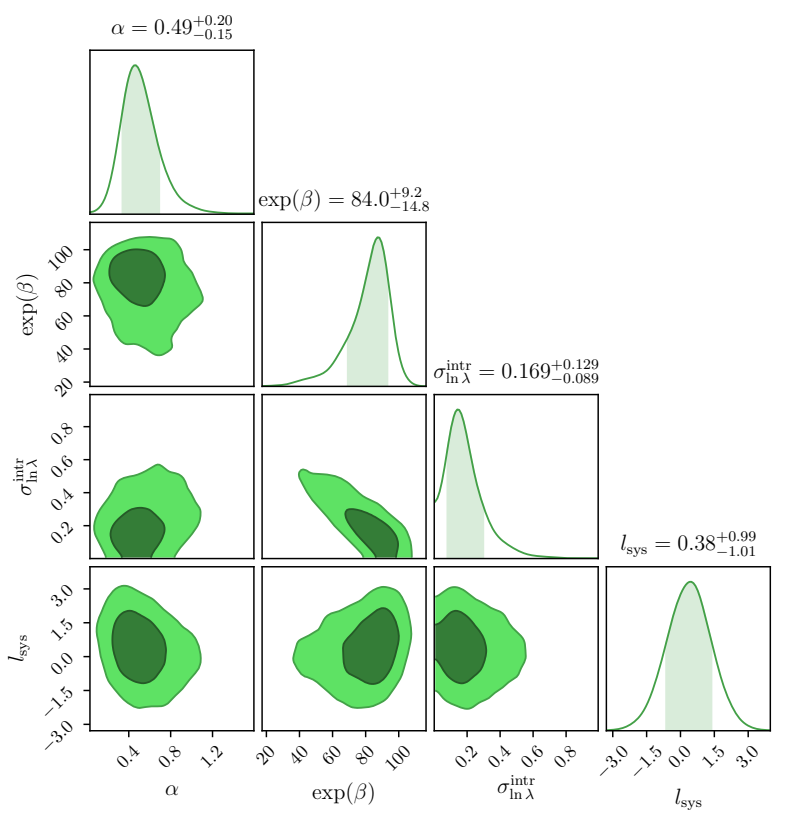

Figure 3. Result from the MCMC fitting, with the one and two dimensional projections of the posterior distributions for the CFHT samples. Contours indicate the statistical $1 \sigma(68 \%)$, and $2 \sigma(95 \%)$ credible regions.

The subscript $i$ is omitted in the normalization as it is identical for all clusters. We note, that the full likelihood function incorporates three of the four selection effects: X-ray selection $P\left(I_{X} \mid \mu_{i}, \tilde{z}_{i}, v_{i}\right)$, to account for covariance between X-ray cluster properties with richness, optical selection $P\left(I_{\mathrm{opt}} \mid v_{i}, \mu_{i}, \theta\right)$, to account for the incompleteness of the SDSS richness, and the sampling function $P\left(I_{\text {samp }} \mid \ln \tilde{\lambda}_{S D S S}\right)$, to account for the fact that we analyse a subsample of the initial CODEX sample. We don't include the fourth selection function, the optical cleaning function $P\left(I_{\text {clean }} \mid \tilde{\lambda}, \tilde{z}\right)$ in the data likelihood, as it is only used to make the redshift dependent cut, removing cluster ID 12451, 18127, and 36818 from the S-I sample.

\section{RESULTS AND DISCUSSION}

We sample the likelihood of the parameters using the EMCEE package (Foreman-Mackey et al. 2013a), which is a Markov Chain Monte Carlo (MCMC) algorithm. We run 24 walkers with 2.000 steps each, excluding the first 400 steps of each chain to remove the burn-in region. We checked the chain convergence by running a successful Gelman-Rubin and Geweke statistic for it using the ChainConsumer package (Hinton 2016). The summary of both initial and prior parameter values used for the MCMC and their posterior values and $1 \sigma$ statistical uncertainties are listed in Table 2 . The initial values for these scaling relations are set to the results of the SPIDERS cluster work (Capasso et al. 2019). Originally, we set the upper limit of $\alpha$ prior to 3, but above 1.6, this upper limit introduced two additional disconnected regions of relatively good likelihood. The two regions had mean values of $\alpha=2.4, \beta=4.4$ and $\sigma_{\ln \lambda}^{\text {intr }}=0.25$, and $\alpha=2.1, \beta=4.2$ and $\sigma_{\ln \lambda}^{\text {intr }}=1.00$. The scaling relations of these two regions have nonphysically low true and mean richness at low masses $\left(<3 \times 10^{14} M_{\odot}\right)$. Therefore, we rerun the MCMC algorithm with the upper limit of $\alpha$ prior set to 1.6, which removed the two nonphysical regions. We report the maximum likelihood of the posterior distribution as our best-fit values, and the uncertainties correspond to the interval containing $68 \%$ of the points.

Fig. 3 shows the results of the MCMC fitting. For the normalization $\lambda_{0}$ of the richness-mass relation, in logarithmic form $\left\langle\ln \lambda \mid \mu_{200 c}\right\rangle=\ln \lambda_{0}+\alpha \mu_{200 c}$, we found $\lambda_{0}=\exp \beta=84.0_{-14.8}^{+9.2}$, and for the slope $\alpha=0.49_{-0.15}^{+0.20}$ at pivot mass $M_{200 \mathrm{c} \text {, piv }}=$ $10^{14.81} M_{\odot}$. Our result for the intrinsic scatter in richness at fixed mass is $\sigma_{\ln \lambda \mid \mu}^{\text {intr }}=0.17_{-0.09}^{+0.13}$.

We compare our richness-mass relation to previous work from Mulroy et al. (2019), Capasso et al. (2019), McClintock et al. (2019), and Bleem et al. (2020). We give a brief summary of each of their results below.

In Mulroy et al. (2019), a simultaneous analysis on several galaxy cluster scaling relations between weak lensing mass and multiple cluster observables is done, including richness-mass relation in logarithmic space $\left\langle\ln \lambda \mid \mu_{500 c}\right\rangle=\beta+\alpha \mu_{500 c}$ using a sample of 41 X-ray luminous clusters from the Local Cluster Substructure Survey (LoCuSS), spanning the redshift range of $0.15<z<0.3$ and mass range of $2.1 \times 10^{14} M_{\odot}<M_{500 c, W L}<1.6 \times 10^{15} M_{\odot}$, with $z_{\text {piv }}=0.22$, and $M_{500 c \text {, piv }}=7.14 \times 10^{14} M_{\odot}$. Their method for estimating the data likelihood function has the same basis as this work, thus we expect the least disagreement between their results and ours.

Capasso et al. (2019) derive the richness-mass-redshift relation $\left\langle\lambda \mid \mu_{200 c}, z\right\rangle=A \mu_{200 c}^{\alpha}\left(\frac{1+z}{1+z_{\text {piv }}}\right)^{\gamma}$ using a sample of $428 \mathrm{X}$ ray luminous clusters from the SPIDERS survey, spanning the redshift range $0.03 \leqslant z \leqslant 0.66$ and dynamical mass range $1.6 \times 10^{14} M_{\odot}<M_{200 c \text {, dyn }}<1.6 \times 10^{15} M_{\odot}$ with $z_{\text {piv }}=0.18$ and $M_{200 c \text {, piv }}=3 \times 10^{14} M_{\odot}$. We compare our richness-mass results to their baseline analysis that accounted for the CODEX selection function. Since the CODEX survey is part of the SPIDERS programme, they share a similar CODEX selection function as we do. Between $0.4<z<0.65$ our CODEX cluster sample overlap with Capasso et al. (2019) with the cluster mass, richness, and redshift range. However, clusters with $z>0.4$ in both Capasso et al. (2019) and our work have the median number of spectroscopic redshift members $\leqslant 20$, as can be seen from Fig. 7 , below, thus the quality of dynamical mass estimates is very different at $z<0.2$, where there are many more than 20 members (median is up to 60 members at $z<0.1$ ).

McClintock et al. (2019) derive mass-richness-redshift relation $\left\langle M_{200 m} \mid \lambda, z\right\rangle=M_{0}(\lambda / 40)^{F}((1+z) / 1.35)^{G}$, and they constrained the normalization of their scaling relation at the 5.0 per cent level, finding $M_{0}=(3.081 \pm 0.075) \times 10^{14} M_{\odot}$ at $\lambda=40$ and $z=0.35$. They find the richness slope at $F=1.356 \pm 0.051$ and the redshift scaling index $G=-0.3 \pm 0.30$. They use redMaPPer galaxy cluster identifier in the Dark Energy Survey Year 1 data using weak gravitational lensing, and $4 \times 3$ bins of richness $\lambda$ and redshift $z$ for $\lambda \geqslant 20$ and $0.2 \leqslant z \leqslant 0.65$. The analysis of McClintock et al. (2019) is the most statistically constraining result from the literature that we consider. However, they consider purely optically selected clusters, which are known to be prone to contamination of low-mass systems.

Bleem et al. (2020) derive richness-mass-redshift relation $\left\langle\ln \lambda \mid M_{500 c}\right\rangle=\ln A+B \ln \left(M_{500 c} / 3 \times 10^{14} M_{\odot} h^{-1}\right)+$ $C \ln (E(z) / E(z=0.6))$, and found $A=76.9 \pm 8.2, B=1.020 \pm 0.08$, $C=0.29 \pm 0.27$. They report finding a $28 \%$ shallower slope $F=1 / B$ than McClintock et al. (2019) with the difference significant at the $4 \sigma$ level. This $2770 \mathrm{deg}^{2}$ survey is conducted using the polarization sensitive receiver in the South Pole Telescope (SPTpol) using the identified Sunyaev-Zel'dovich (SZ) signal of 652 clusters to 
Table 2. Summary of measured parameters, their initial values, priors and posteriors. The initial parameter values for each of the 24 random walkers in the MCMC run are randomly drawn around a circle with the center value listed in the Initial column and with radius $10^{-2}$. This way all walkers start to scan the parameter space at slightly different initial position.

\begin{tabular}{cccc}
\hline \hline Parameter & Initial & Prior & Posterior \\
\hline \hline$\alpha$ & 0.98 & flat $(0,1.6)$ & $0.49_{-0.15}^{+0.20}$ \\
$\beta$ & 3.68 & flat $(0,6)$ & $4.42_{-0.20}^{+0.13}$ \\
$\sigma_{\ln \lambda}^{\text {intr }}$ & 0.22 & flat $(0,1)$ & $0.17_{-0.09}^{+0.13}$ \\
$l_{\text {sys }}^{+0.0}$ & 0.0 & $\mathcal{N}[0,1]$ & $0.38_{-1.01}^{+0.99}$ \\
\hline
\end{tabular}

$\alpha$ is the mass slope of the richness-mass relation $\langle\ln \lambda \mid \mu\rangle=\alpha \mu+\beta$.

$\beta$ is intercept (normalization) of the richness-mass relation.

$\sigma_{\ln \lambda}^{\text {intr }}$ is the intrinsic scatter in richness, which quantifies how much true richness at given mass scatters from the mean.

$l_{\text {sys }}$ is a scalar lensing systematic parameter. It is used to draw how different the noiseless log lensing masses are from the log true masses due to imperfect calibration of lensing shapes, redshifts, and the cluster density profiles.

Table 3. Scaling relation parameter comparison to literature. The credible intervals refers to $1 \sigma(68 \%)$ statistical uncertainties.

\begin{tabular}{cccc}
\hline \hline Bayesian analysis results & $\begin{array}{c}\text { Intercept } \\
\lambda_{0}=\exp (\beta)\end{array}$ & $\begin{array}{c}\text { Slope } \\
\alpha\end{array}$ & $\begin{array}{c}\text { Scatter } \\
\sigma_{\ln \lambda}^{\text {intr }}\end{array}$ \\
\hline CODEX lensing sample & $84.0_{-14.8}^{+9.2}$ & $0.49_{-0.15}^{+0.20}$ & $0.17_{-0.09}^{+0.13}$ \\
\hline \hline Previously published results & $\lambda_{0}\left(10^{14.81} M_{\odot}, z=0.5\right)$ & $M_{200 c}^{\alpha}$ & $\sigma_{\ln \lambda}^{\text {intr }}$ \\
\hline LoCuSS prediction (Mulroy et al. 2019) & $93.66 \pm 7.43$ & $0.74 \pm 0.06$ & $0.24 \pm 0.05$ \\
SPIDERS prediction (Capasso et al. 2019) & $65.10 \pm 7.21$ & $0.98 \pm 0.07$ & $0.22_{-0.09}^{+0.08}$ \\
SPTpol prediction (Bleem et al. 2020) & $79.15 \pm 8.30$ & $1.02 \pm 0.08$ & $0.23 \pm 0.16$ \\
DES Y1 prediction (McClintock et al. 2019) & $70.66 \pm 2.55$ & $0.73 \pm 0.03$ & - \\
\hline \hline
\end{tabular}

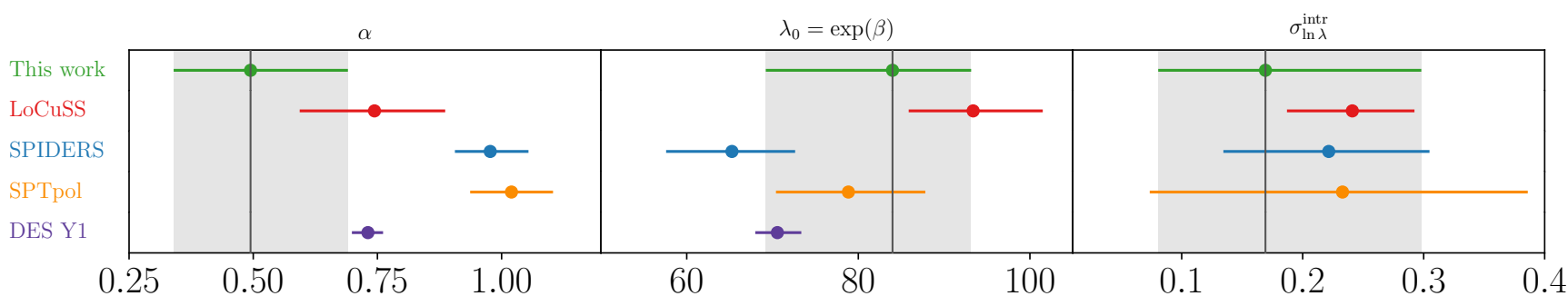

Figure 4. Comparison between the predicted richness and other results from the literature. The predicted richnesses are evaluated at $M_{200 c}=10^{14.81} M_{\odot}$ and $z=0.5$. Gray bands denote the statistical $1 \sigma(68 \%)$ uncertainty of this work. For the DES Y1 analysis, the intrinsic scatter and its $1 \sigma$ uncertainty is not shown, as it is not constrained in their work.

estimate the cluster masses. The richnesses of the clusters are estimated using the redMaPPer algorithm and matched with DES Y3 RM catalog to calibrate the richness-mass relation, taking the SPT selection into account. This sample is closest to ours in terms of sample definition, as both X-ray and SZ signal require the presence of hot intracluster medium (ICM), which cleans the contamination of optical samples.

In a recently published CODEX weak lensing analysis by Phriksee et al. (2020), a mass-richness comparison was made to Capasso et al. (2019), with 279 clusters in the optical richness range at $20 \leqslant \lambda \leqslant 110$, and $0.1 \leqslant z \leqslant 0.2$. They found an excellent agreement with both dynamical mass estimates and weak lensing mass estimates at $z \leqslant 0.15$.

We use the colossus python package (Diemer 2018) to convert the $M_{500 c}$, and $M_{200 m}$ to $M_{200 c}$ when necessary, and evaluate the slope and intercept at $M_{200 c \text {, piv }}=10^{14.81} M_{\odot}$, in order to com- pare our constraints with other results. Since Capasso et al. (2019), McClintock et al. (2019), and Bleem et al. (2020) included the $z$ evolution of their scaling relation, we estimate their relation at $z=0.5$, the mean $z$ of our 25 cluster subsample, to make our results comparable. For Mulroy et al. (2019), we rescale the scaling relation parameters by assuming $\lambda_{0}(z)=\exp \beta(z)=$ const. For the McClintock et al. (2019) results, we use the Leauthaud et al. (2009) to invert the mass-richness relation, and evaluate the relation at $z=0.5$, $M_{200 \mathrm{c}, \text { piv }}=10^{14.81} M_{\odot}$. The inversion requires a bias term, which depends on the $\sigma_{\ln \lambda}^{\mathrm{intr}}$, for which we use our intrinsic scatter value of $\sigma_{\ln \lambda}^{\text {intr }}=0.17_{-0.09}^{+0.13}$, as McClintock et al. (2019) did not constrain it. In Table 3, we show the predicted richness-mass mean parameter values and their $1 \sigma$ statistical uncertainties from the LoCuSS, SPIDERS, SPTpol, and DES Y1 work, all evaluated at $z=0.5$ and $M_{200 c \text {, piv }}=10^{14.81} M_{\odot}$. In Fig. 4, we compare the slope and 


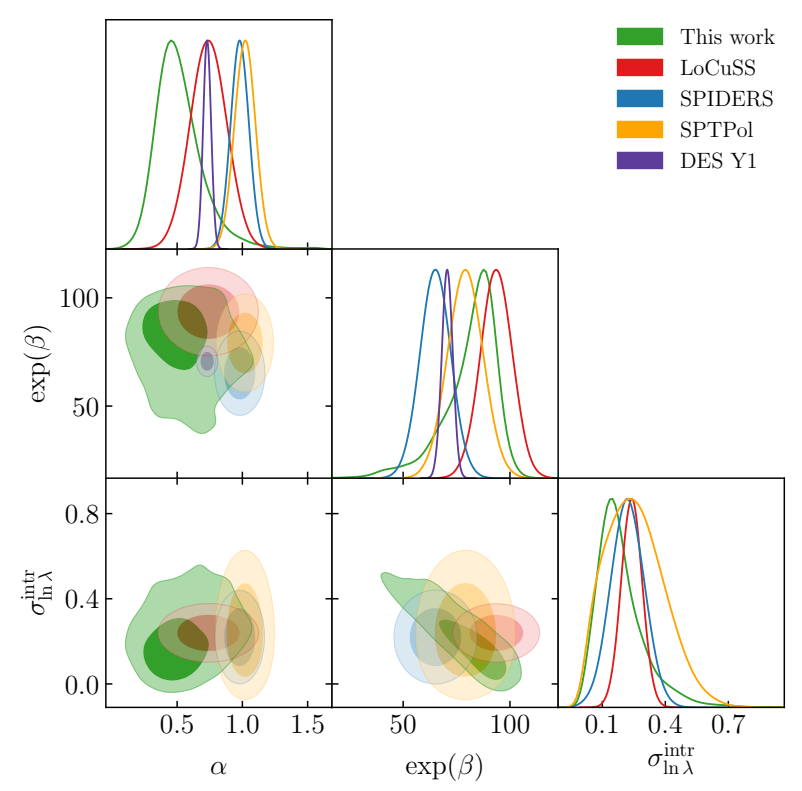

Figure 5. Identical MCMC fitting results as in Fig. 3, but with the inclusion of the scaling relation results from the literature, rescaled at $M_{200 c}=10^{14.81} M_{\odot}$ and $z=0.5$. Contours indicate the statistical $1 \sigma$ $(68 \%)$, and $2 \sigma(95 \%)$ credible regions.

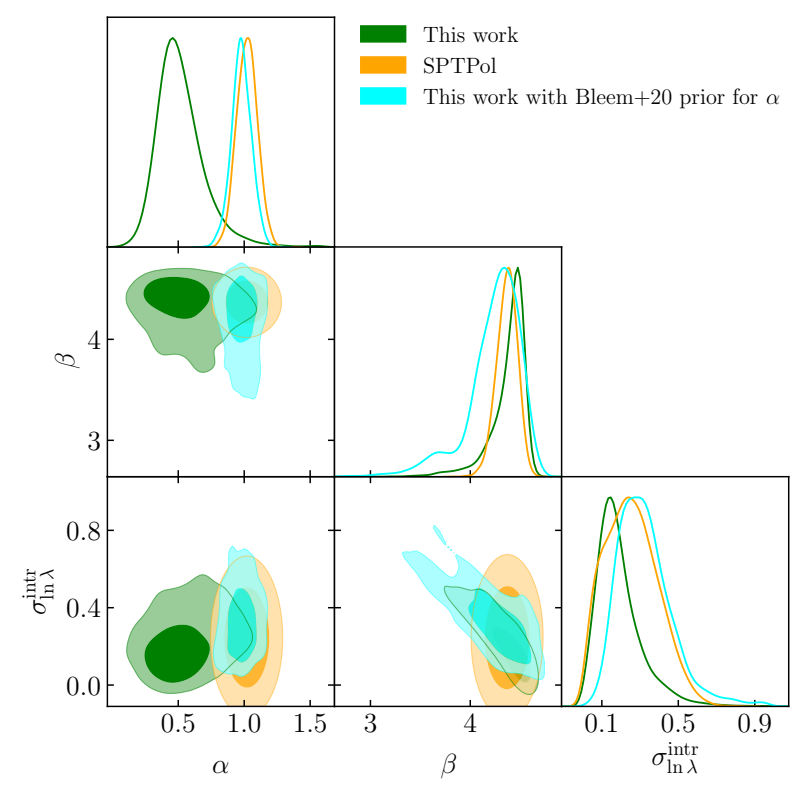

Figure 6. Comparison of the predicted Bleem et al. (2020) parameter distributions (in orange) with respect to this work, but assuming a similar slope as in Bleem et al. (2020) (in cyan). For the slope, instead of using a flat prior, we use a Gaussian prior with the mean and scatter set to SPTpol prediction listed in Table 3. Contours indicate the statistical $1 \sigma(68 \%)$, and $2 \sigma(95 \%)$ credible regions.

predicted richness $\lambda_{0}=\left\langle\lambda \mid M=10^{14.81} M_{\odot}, z=0.5,\right\rangle=\exp (\beta)$ from our work (gray bands) to the ones in the literature.

Fig. 5 shows the predicted mean relations from Table 3 overplotted to our MCMC fitting results from Fig. 3. We note that all the predicted mean results fall within $2 \sigma$ region of our posterior distributions, where the largest deviation in both slope and intercept is with Capasso et al. (2019) and Bleem et al. (2020).

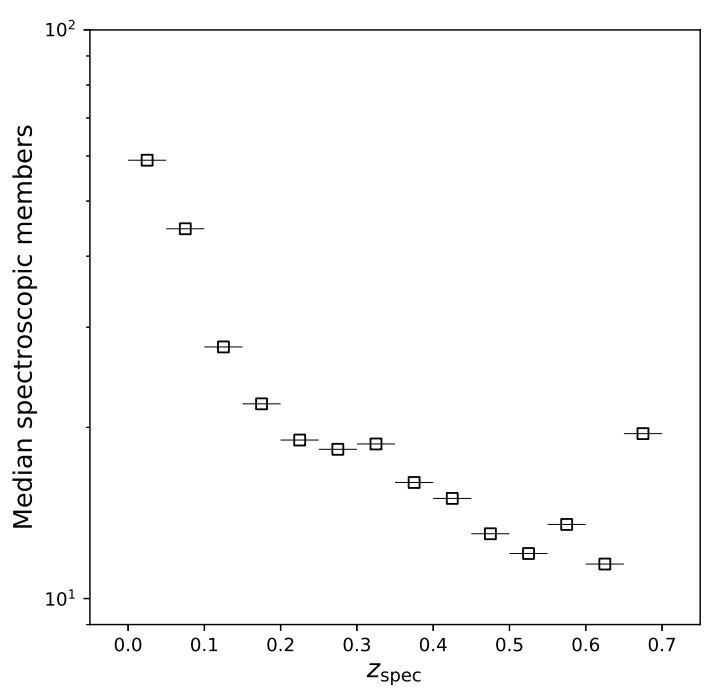

Figure 7. Median of the spectroscopic members as a function of spectroscopic redshift of the SPIDERS sample, which CODEX sample is part of. The redshift bin is set to $\Delta z=0.05$, and the selection cuts are set those of Capasso et al. (2019) $\left(\lambda \geqslant 60\right.$ and $\left.N_{\text {mem }} \geqslant 10\right)$.

Since our slope is only accurate up to $2 \sigma$ for both Capasso et al. (2019) and Bleem et al. (2020), with both centered around unity, and the latter having shallower constraints for the slope, to see how different prior of the slope affects our parameter estimation, we redo our Bayesian analysis with the same 25 clusters as before, but using a Gaussian prior for the slope, set to the mean and the scatter from SPTpol prediction of Table 3. In Fig. 6, we show the posterior distributions of the Gaussian prior for the slope in cyan, and compare the parameter distributions against the predicted SPTpol parameter distributions, shown in orange. When using a Gaussian prior for the slope, we found the posterior slope $\alpha=0.98 \pm 0.09$, normalization $\lambda_{0}=\exp (\beta)=74.4_{-18.2}^{+21.4}$, and intrinsic scatter in richness $\sigma_{\ln \lambda}^{\text {intr }}=0.28_{-0.14}^{+0.16}$. We create the SPTpol parameter distributions by using a multivariate Gaussian with mean and elements of the diagonal scatter matrix set at the mean and the square of the $1 \sigma$ uncertainties of the SPTpol predictions from Table 3. We note that a tight parameter constraint on the slope loosens both the normalization, and the intrinsic scatter to wider range, forcing the mean of the normalization parameters towards smaller values, but intrinsic scatter towards the predicted SPTpol results. Since the number of clusters is small in our subsample, the prior shape has a larger impact on the final marginalized posterior distributions. We have a preference for choosing a flat prior for the slope, as our data points are within narrow mass range with large uncertainty on the mass, and small uncertainty on the richness.

In Fig. 8, we show the richness-mass relations from Table 3 . In the upper panel, we only consider the statistical $1 \sigma(68 \%)$ uncertainty around the mean relations, whereas in the lower panel, we consider the $1 \sigma(68 \%)$ interval, where new richness observations may fall at fixed mass. We do this by introducing the $\sigma_{\ln \lambda}^{\text {intr }}$ and its $1 \sigma$ uncertainty to all surveys, except for DES Y1, which lacked intrinsic scatter information. The $1 \sigma$ confidence regions in Fig. 8 are done the following way:

(i) Draw 5000 new scaling relation parameter samples $(\alpha, \beta$, 
and $\sigma_{\ln \lambda}^{\text {intr }}$ ) from a multivariate Gaussian distribution with mean and diagonal scatter matrix set to results from Table 3,

(ii) Use new values of $\alpha$ and $\beta$ to generate 5000 new mean richnesses at each mass point,

(iii) For the upper panel, calculate the $1 \sigma$ statistics of these 5000 mean richness values and plot them,

(iv) For the lower panel, sample 1000 new richness values for each of the 5000 mean richness values from a log-normal distribution with mean and scatter set to values sampled from the multivariate Gaussian in step (i),

(v) Calculate the $1 \sigma$ uncertainty from the 1000 new richness values for each of the 5000 mean richnesses and plot those uncertainties to the lower panel.

The error envelopes in the lower panel include the $1 \sigma$ uncertainties of the slope, the intercept and the intrinsic scatter in richness. Typically in the literature, only the mean with $1 \sigma$ uncertainties are shown as the scaling relation, like in the upper panel of Fig. 8, but this method only accounts for uncertainty in the slope and intercept, and does not consider that the mean relation may deviate from the fixed data points by the intrinsic scatter. In the lower panel of Fig. 8, we also take account the effect of intrinsic scatter in richness and its $1 \sigma$ uncertainty in the scaling relations. The latter method takes into account both the uncertainty of the mean relation due to intrinsic scatter, along with the uncertainty on the parameters. We note that the data points in Fig. 8 refer to observed values from Table 1, not to their true values. We show these here to point out the narrow mass range of the observed data with large statistical uncertainty in weak lensing mass and small uncertainty in the observed richness.

From Fig. 4, the richness normalization $\lambda_{0}$, at $z=0.5$ and $M_{200 c}=10^{14.81} M_{\odot}$, from our work overlaps within $1 \sigma$ uncertainty with all four different survey richness normalizations that we consider. The main difference in the normalization is between LoCuSS, which had measured clusters at $0.15<z<0.3$, and the rest of the surveys, but given that LoCuSS richness relation is estimated without redshift dependent evolution in richness, so this might mean that there is an evolution of cluster richness at a given mass, as discussed in (Capasso et al. 2019).

Relatively flat slopes found in this and in LoCuSS work could be attributed to a combination of probing small mass range, and that intrinsic scatter in richness could increase with decreasing mass $\sigma_{\ln \lambda}^{\text {intr }}(m) \propto 1 / m$. Although, our mass slope is only $1 \sigma$ away from the slope found by McClintock et al. (2019), a steeper slope of $\alpha=1.0_{-0.22}^{+0.22}$ was robustly established in low-z CODEX studies (Phriksee et al. 2020), and was attributed to CODEX X-ray clusters being less prone to possible contamination by projected low mass groups of galaxies along line-of-sight than purely optically selected clusters, such as McClintock et al. (2019).

Also, from Fig. 4, we see that our result on the intrinsic scatter in richness overlaps within $1 \sigma$ with other results found from the literature, however with smaller mean at $\sigma_{\ln \lambda}^{\text {intr }}=0.17_{-0.09}^{+0.13}$. When the same analysis is done with a Gaussian prior on the slope, $\alpha \sim$ $\mathcal{N}(1.02,0.08)$ (see Fig. 6), we find the intrinsic scatter at $\sigma_{\ln \lambda}^{\text {intr }}=$ $0.28_{-0.14}^{+0.16}$, indicating the importance of the prior choice, when a small sample size is considered.

Our comparison to the results of the dynamical mass modelling, presented in Capasso et al. (2019), indicate marginally lower mass for a given richness at richness values around 80 . Considering other weak lensing calibrations, performed on X-ray clusters, we quote from Phriksee et al. (2020) that at $z<0.15$ the weak lensing calibration of CODEX clusters of Phriksee et al. (2020) agrees well with Capasso et al. (2019), while we find from Fig. 4 that LoCuSS
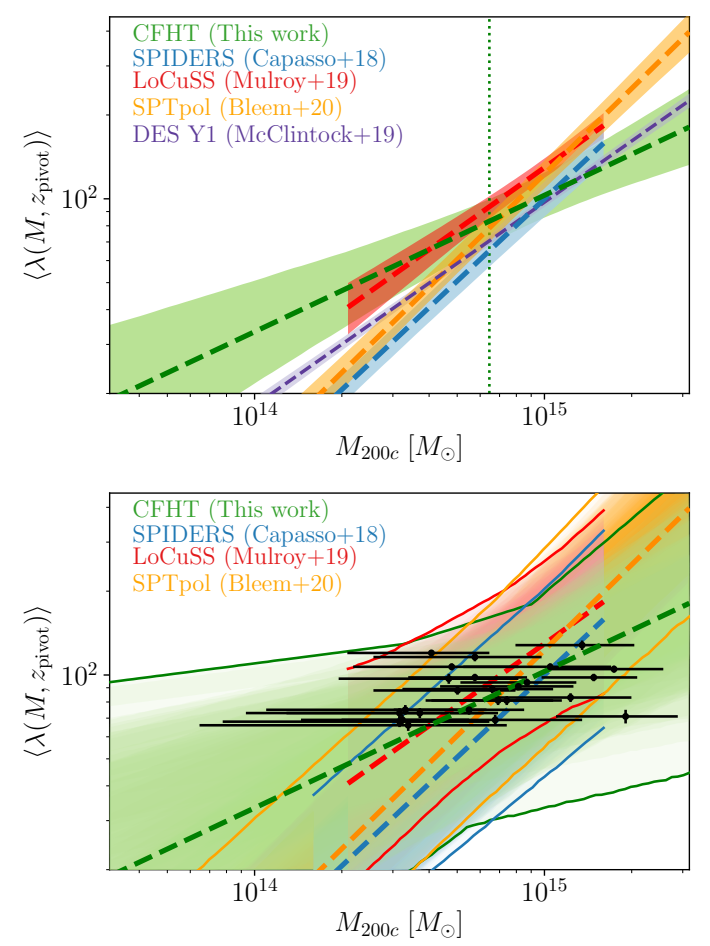

Figure 8. Upper panel: Mean relation comparison with the predicted results from the literature. The confidence regions (light blue, light green, light red, light orange, and light violet envelopes) represent the $1 \sigma$ uncertainty of the slope and intercept of the mean relations (blue, green, red, orange and violet dashed lines, respectively). The predicted relations from DES Y1, SPTpol, and SPIDERS have been scaled to $z_{\text {pivot }}=0.5$, and the DES Y1 relation is inverted according to Leauthaud et al. (2009). The vertical green line is the pivot mass of this work. We limit each predicted relation to their respective mass and richness range.

Lower panel: Since in the data likelihood function, we account for the intrinsic scatter in richness, it is meaningful to include its effect to the overall parameter uncertainty budget. The error envelopes takes into account the $1 \sigma$ uncertainties of the slope, intercept and the intrinsic scatter in richness. The uncertainties in data points represent $1 \sigma$ statistical error in mass and observed richness.

(Mulroy et al. 2019) results $(0.15<z<0.3)$ are in significant tension with Capasso et al. (2019). These results, if confirmed, could be used to constrain the models of modified gravity (Arnold et al. 2014; Sakstein et al. 2016; Wilcox et al. 2016; Mitchell et al. 2018; Tamosiunas et al. 2019). Improvements in spectroscopic follow-up of high-z clusters is however, very critical. As Zhang et al. (2017) showed, a low number of spectroscopic redshifts per cluster and fiber-collisions of SPIDERS tiling can have strong effect on bias and scatter of dynamical mass estimates.

\section{CONCLUSIONS}

We present the results of Bayesian weak lensing mass calibration analysis of CODEX cluster sample of 25 clusters for high redshift $(0.35<z<0.62)$, with redMaPPer richness $\geqslant 60$, and with a detailed consideration of systematic uncertainties. The weak lensing data is obtained by pointed CFHT observations of CODEX clusters, to which we add a reanalysis of the public CFHTLS data. We obtain the cluster masses by running a likelihood analysis including a covariance matrix to account for contributions by large scale structure and intrinsic properties. We refine the original richness estimates 
based on SDSS photometry by rerunning redMaPPer on CFHT photometry and obtain richness-mass relation $\langle\ln \lambda \mid \mu\rangle=\alpha \mu+\beta$, with $\mu=\ln \left(M_{200 c} / 10^{14.81} M_{\odot}\right)$, and compare this relation to the one obtained by Mulroy et al. (2019) $(z \sim 0.2)$, and $\mathrm{z}=0.5$ predictions of Capasso et al. (2019), McClintock et al. (2019), and Bleem et al. (2020). We measure richness-mass relation with slope of $\alpha=0.49_{-0.15}^{+0.20}$ and intercept of $\lambda_{0}=\exp (\beta)=84.0_{-14.8}^{9.2}$, using a data likelihood function that incorporate the overall error budget of the weak lensing mass calibration analysis, along with optical, $\mathrm{X}$-ray, survey incompleteness and subsample selection effects.

We find our results on the slope, intercept, and intrinsic scatter in richness overlap with the weak lensing analysis of low-z $(0.15<z<0.3)$ LoCuSS clusters by Mulroy et al. (2019) within $1 \sigma$ uncertainty over the entire LoCuSS mass range.

At masses of $10^{14.81} M_{\odot}$, our $68 \%$ credible region for the mean cluster richness overlaps with that of Mulroy et al. (2019), McClintock et al. (2019), and Bleem et al. (2020), and at around the 16th percentile, slightly overlaps the 84th percentile of the Capasso et al. (2019). The $1 \sigma$ statistical uncertainty in richness is at the level of difference in the results based on different cluster selection and different mass measurements. Even though we consider a multitude of selection effects with a narrow mass range and a small sample size, we find relatively flat slope. Thus, future improvements should not be directed solely towards increasing the sample size, but also on understanding the selection effects and improvements in the mass measurements. The importance of our work consists in extending the weak lensing calibration of massive X-ray clusters to $z \leqslant 0.6$, where previously, large disagreements on weak lensing calibrations were reported (Smith et al. 2016).

\section{ACKNOWLEDGEMENTS}

We thank an anonymous referee for thorough review of the manuscript, Raffaella Capasso and Jacob Ider Chitham for discussion of the results. This work is based on observations obtained with MegaPrime/MegaCam, a joint project of CFHT and CEA/IRFU, at the Canada-France-Hawaii Telescope (CFHT) which is operated by the National Research Council (NRC) of Canada, the Institut National des Science de l'Univers of the Centre National de la Recherche Scientifique (CNRS) of France, and the University of Hawaii.

We use data from the Canada-France-Hawaii Lensing Survey (Heymans et al. 2012), hereafter referred to as CFHTLenS. The CFHTLenS survey analysis combined weak lensing data processing with THELI (Erben et al. 2013) and shear measurement with lensfit (Miller et al. 2013). A full systematic error analysis of the shear measurements in combination with the photometric redshifts is presented in Heymans et al. (2012).

Based on observations made with the Nordic Optical Telescope, operated by the Nordic Optical Telescope Scientific Association at the Observatorio del Roque de los Muchachos, La Palma, Spain, of the Instituto de Astrofisica de Canarias.

We acknowledge Fabrice Brimioulle for his substantial work on an early version of this manuscript, and we understand his decision not to be listed on the paper, since he is no longer working in astronomy. We thank Matthew R. Becker and Andrey Kravtsov for making their cluster simulations available.

KK and JV acknowledge financial support from the Finnish Cultural Foundation, KK the Magnus Ehrnrooth foundation, and the Academy of Finland grant 295113. This work was supported by the Department of Energy, Laboratory Directed Research and Develop- ment program at SLAC National Accelerator Laboratory, under contract DE-AC02-76SF00515 and as part of the Panofsky Fellowship awarded to DG. NC acknowledges financial support from the Brazilian agencies CNPQ and CAPES (process \#2684/2015-2 PDSE). NC also acknowledges support from the Max-Planck-Institute for Extraterrestrial Physics and the Excellence Cluster Universe. ESC acknowledges financial support from Brazilian agencies CNPQ and FAPESP (process \#2014/13723-3). LM acknowledges STFC grant ST/N000919/1. AF \& CK acknowledge the Finnish Academy award, decision 266918. HYS acknowledges the support from the Shanghai Committee of Science and Technology grant No. 19ZR1466600. We acknowledge R. Bender for the use of his photometric redshift pipeline in this work. NC acknowledge J. Weller for the hospitality.

This work made use of the astronomical data analysis software TOPCAT (Taylor 2005). Data analysis has been carried out with University of Helsinki computing clusters Alcyone and Kale. We acknowledge the use of the research infrastructures Euclid Science Data Center Finland (SDC-FI, urn:nbn:fi:research-infras2016072529) and the Finnish Grid and Cloud Computing Infrastructure (FGCI, urn:nbn:fi:research-infras-2016072533), and the Academy of Finland infrastructure grant 292882. The author acknowledges the usage of the following python packages, in alphabetical order: astropy (Astropy Collaboration et al. 2013; The Astropy Collaboration et al. 2018), chainConsumer (Hinton 2016), emcee (Foreman-Mackey et al. 2013b), matplotlib (Hunter 2007), numpy (Oliphant 06 ; van der Walt et al. 2011), and scipy (Virtanen et al. 2019).

\section{DATA AVAILABILITY}

The raw data underlying this article are available in CFHT server, at https://www.cadc-ccda.hia-iha.nrc-cnrc.gc.ca/en/ cfht/

\section{REFERENCES}

Allen S. W., Evrard A. E., Mantz A. B., 2011, ARA\&A, 49, 409

Applegate D. E., et al., 2014, MNRAS, 439, 48

Arnold C., Puchwein E., Springel V., 2014, MNRAS, 440, 833

Astropy Collaboration et al., 2013, A\&A, 558, A33

Becker M. R., Kravtsov A. V., 2011, ApJ, 740, 25

Benson B. A., et al., 2013, ApJ, 763, 147

Bertin E., Arnouts S., 1996, A\&AS, 117, 393

Bertin E., Mellier Y., Radovich M., Missonnier G., Didelon P., Morin B., 2002, in Bohlender D. A., Durand D., Handley T. H., eds, Astronomical Society of the Pacific Conference Series Vol. 281, Astronomical Data Analysis Software and Systems XI. p. 228

Bielby R., et al., 2012, A\&A, 545, A23

Bleem L. E., et al., 2015, ApJS, 216, 27

Bleem L. E., et al., 2020, The Astrophysical Journal Supplement Series, 247,25

Böhringer H., et al., 2004, A\&A, 425, 367

Boller T., Freyberg M. J., Trümper J., Haberl F., Voges W., Nandra K., 2016, A\&A, 588, A103

Boulade O., et al., 2003, in M. Iye \& A. F. M. Moorwood ed., Society of Photo-Optical Instrumentation Engineers (SPIE) Conference Series Vol. 4841, Society of Photo-Optical Instrumentation Engineers (SPIE) Conference Series. pp 72-81, doi:10.1117/12.459890

Brimioulle F., Seitz S., Lerchster M., Bender R., Snigula J., 2013, MNRAS, 432, 1046

Capasso R., et al., 2019, MNRAS, 486, 1594

Cibirka N., et al., 2016, preprint, (arXiv:1612.06871)

Clerc N., et al., 2016, MNRAS, 463, 4490 
Diemer B., 2018, ApJS, 239, 35

Dietrich J. P., Erben T., Lamer G., Schneider P., Schwope A., Hartlap J., Maturi M., 2007, A\&A, 470, 821

Duffy A. R., Schaye J., Kay S. T., Dalla Vecchia C., 2008, Mon. Not. Roy. Astron. Soc., 390, L64

Dutton A. A., Macciò A. V., 2014, MNRAS, 441, 3359

Ebeling H., Edge A. C., Bohringer H., Allen S. W., Crawford C. S., Fabian A. C., Voges W., Huchra J. P., 1998, MNRAS, 301, 881

Ebeling H., Edge A. C., Mantz A., Barrett E., Henry J. P., Ma C. J., van Speybroeck L., 2010, MNRAS, 407, 83

Eisenstein D. J., Hu W., 1998, ApJ, 496, 605

Erben T., et al., 2005, Astronomische Nachrichten, 326, 432

Erben T., et al., 2009, A\&A, 493, 1197

Erben T., et al., 2013, MNRAS, 433, 2545

Farahi A., et al., 2019, Nature Communications, 10

Fenech Conti I., Herbonnet R., Hoekstra H., Merten J., Miller L., Viola M., 2017, MNRAS, 467, 1627

Finoguenov A., et al., 2020, CODEX clusters. Survey, catalog, and cosmology of the X-ray luminosity function (arXiv: 1912.03262), doi:10.1051/0004-6361/201937283

Foreman-Mackey D., Hogg D. W., Lang D., Goodman J., 2013a, PASP, 125, 306

Foreman-Mackey D., Hogg D. W., Lang D., Goodman J., 2013b, PASP, 125, 306

Giles, P. A. et al., 2016, A\&A, 592, A3

Gladders M. D., Yee H. K. C., 2005, ApJS, 157, 1

Górski K. M., Hivon E., Banday A. J., Wandelt B. D., Hansen F. K., Reinecke M., Bartelmann M., 2005, ApJ, 622, 759

Gozaliasl G., et al., 2014, A\&A, 566, A140

Gozaliasl G., et al., 2019, MNRAS, 483, 3545

Gruen D., Brimioulle F., 2016, preprint, (arXiv: 1610.01160)

Gruen D., Bernstein G. M., Lam T. Y., Seitz S., 2011, MNRAS, 416, 1392

Gruen D., et al., 2013, MNRAS, 432, 1455

Gruen D., et al., 2014, MNRAS, 442, 1507

Gruen D., Seitz S., Becker M. R., Friedrich O., Mana A., 2015, Mon. Not. Roy. Astron. Soc., 449, 4264

Henry J. P., Evrard A. E., Hoekstra H., Babul A., Mahdavi A., 2009, ApJ, 691,1307

Herbonnet R., et al., 2019, arXiv e-prints, p. arXiv:1912.04414

Heymans C., et al., 2012, MNRAS, 427, 146

Hildebrandt H., et al., 2017, MNRAS, 465, 1454

Hinton S., 2016, The Journal of Open Source Software, 1, 45

Hoekstra H., 2001, A\&A, 370, 743

Hoekstra H., 2003, MNRAS, 339, 1155

Hoekstra H., 2007, MNRAS, 379, 317

Huff E., Mandelbaum R., 2017, preprint, (arXiv:1702.02600)

Hunter J. D., 2007, Computing In Science \& Engineering, 9, 90

Jarvis M., et al., 2016, MNRAS, 460, 2245

Kannawadi A., et al., 2019, Astronomy \& Astrophysics, 624, A92

Kodwani D., Alonso D., Ferreira P., 2019, The Open Journal of Astrophysics, 2,3

Koester B. P., et al., 2007, ApJ, 660, 221

Komatsu E., et al., 2011, The Astrophysical Journal Supplement Series, 192, 18

Laigle C., et al., 2016, ApJS, 224, 24

Leauthaud A., et al., 2009, The Astrophysical Journal, 709, 97-114

Lerchster M., et al., 2011, MNRAS, 411, 2667

Lieu M., et al., 2016, A\&A, 592, A4

Limber D. N., 1954, ApJ, 119, 655

Mandelbaum R., et al., 2015, MNRAS, 450, 2963

Mantz A., Allen S. W., Rapetti D., Ebeling H., 2010, MNRAS, 406, 1759

Mantz A. B., et al., 2015, MNRAS, 446, 2205

Marrone D. P., et al., 2012, ApJ, 754, 119

McClintock T., et al., 2019, MNRAS, 482, 1352

Melchior P., Viola M., 2012, MNRAS, 424, 2757

Melchior P., et al., 2016, preprint, (arXiv: 1610.06890)

Miller L., et al., 2013, MNRAS, 429, 2858

Mitchell M. A., He J.-h., Arnold C., Li B., 2018, MNRAS, 477, 1133
Mulroy S. L., et al., 2019, Mon. Not. Roy. Astron. Soc., 484, 60

Nagai D., Vikhlinin A., Kravtsov A. V., 2007, ApJ, 655, 98

Nagarajan A., et al., 2018, Monthly Notices of the Royal Astronomical Society, 488, 1728-1759

Navarro J. F., Frenk C. S., White S. D. M., 1996, ApJ, 462, 563

Navarro J. F., Frenk C. S., White S. D. M., 1997, ApJ, 490, 493

Oliphant T., 2006-, NumPy: A guide to NumPy, USA: Trelgol Publishing, http: //www. numpy.org/

Phriksee A., Jullo E., Limousin M., Shan H., Finoguenov A., Komonjinda S., Wannawichian S., Sawangwit U., 2020, MNRAS, 491, 1643

Pickles A. J., 1998, PASP, 110, 863

Planck Collaboration et al., 2016, A\&A, 594, A27

Press W. H., Schechter P., 1974, ApJ, 187, 425

Rasia E., et al., 2012, New Journal of Physics, 14, 055018

Refregier A., Kacprzak T., Amara A., Bridle S., Rowe B., 2012, MNRAS, 425,1951

Rozo E., et al., 2009, The Astrophysical Journal, 703, 601

Rozo E., et al., 2010, ApJ, 708, 645

Rykoff E., et al., 2012, The Astrophysical Journal, 746, 178

Rykoff E. S., et al., 2014, ApJ, 785, 104

Rykoff E. S., et al., 2016, ApJS, 224, 1

Sakstein J., Wilcox H., Bacon D., Koyama K., Nichol R. C., 2016, J. Cosmology Astropart. Phys., 2016, 019

Saro A., et al., 2015, MNRAS, 454, 2305

Schirmer M., 2013, ApJS, 209, 21

Schneider P., van Waerbeke L., Jain B., Kruse G., 1998, MNRAS, 296, 873

Sheldon E. S., Huff E. M., 2017, preprint, (arXiv: 1702.02601)

Sheldon E. S., et al., 2004, AJ, 127, 2544

Sheth R. K., Tormen G., 1999, MNRAS, 308, 119

Simet M., McClintock T., Mandelbaum R., Rozo E., Rykoff E., Sheldon E., Wechsler R. H., 2016, preprint, (arXiv: 1603.06953)

Smith R. E., et al., 2003, MNRAS, 341, 1311

Smith G. P., et al., 2016, MNRAS, 456, L74

Spinelli P. F., Seitz S., Lerchster M., Brimioulle F., Finoguenov A., 2012, MNRAS, 420, 1384

Sunyaev R. A., Zeldovich I. B., 1980, ARA\&A, 18, 537

Tamosiunas A., Bacon D., Koyama K., Nichol R. C., 2019, Journal of Cosmology and Astroparticle Physics, 2019, 053-053

Taylor M. B., 2005, in Shopbell P., Britton M., Ebert R., eds, Astronomical Society of the Pacific Conference Series Vol. 347, Astronomical Data Analysis Software and Systems XIV. p. 29

The Astropy Collaboration et al., 2018, preprint, (arXiv: 1801.02634)

Tinker J., Kravtsov A. V., Klypin A., Abazajian K., Warren M., Yepes G., Gottlöber S., Holz D. E., 2008, ApJ, 688, 709

Umetsu K., Broadhurst T., Zitrin A., Medezinski E., Coe D., Postman M., 2011, ApJ, 738, 41

Vikhlinin A., et al., 2009, ApJ, 692, 1060

Virtanen P., et al., 2019, arXiv e-prints, p. arXiv:1907.10121

Voges W., et al., 1999, A\&A, 349, 389

Wilcox H., Nichol R. C., Zhao G.-B., Bacon D., Koyama K., Romer A. K., 2016, MNRAS, 462, 715

Wright C. O., Brainerd T. G., 2000, ApJ, 534, 34

Zhang Y.-Y., et al., 2017, A\&A, 599, A138

van der Walt S., Colbert S. C., Varoquaux G., 2011, Computing in Science and Engineering, 13, 22

von der Linden A., et al., 2014a, MNRAS, 439, 2

von der Linden A., et al., 2014b, MNRAS, 443, 1973

\section{APPENDIX A: SYSTEMATIC UNCERTAINTIES}

Our lensing signal is affected by two sources of systematics: the errors from shape measurements and distance estimates computed using the colour-magnitude decision tree. The systematic uncertainties enter in our lensing model as a factor that multiplies the theoretical density profile, changing its amplitude to assimilate the errors. This factor follows a Gaussian prior with the mean shifted by 
the bias from both shear and photometric redshift measurements, $1-\delta_{\mathrm{cm}}-\delta_{\mathrm{sm}}$, and width corresponding to the quadratic sum of the variances $\sigma_{\mathrm{cm}}$ and $\sigma_{\mathrm{sm}}$. In the following sections we describe how we derive these contributions from shape and distance measurements.

\section{A1 Shear bias}

As mentioned in subsection 2.5, we expect the residual uncertainty in the lensfit shape measurement to be in the order of 2 per cent (see Fenech Conti et al. 2017) and assume the same uncertainty in case of CFHTLenS lensfit shapes after applying the corrections shown in equations $\mathrm{C} 1$ and $\mathrm{C} 2$. We account for this uncertainty by introducing a shear calibration factor with mean $\delta_{\mathrm{sm}}=0$ and Gaussian width $\sigma_{\mathrm{sm}}=0.02$ in our modelling.

\section{A2 Bias of source redshift distribution}

The colour-magnitude decision tree method contributes to the final error budget through two sources of systematic uncertainties: cosmic variance and errors in the reference catalogue of photometric redshifts. We assess the contribution from photo- $z$ errors by comparing the values of $\beta$ from the CFHTLS D2 field $\left(\beta_{\mathrm{D} 2}\right)$ and $\operatorname{COSMOS} 2015\left(\beta_{\mathrm{C} 2015}\right)$ catalogues. The difference in $\beta$ from this matched catalogue is free of cosmic variance because we use different template fits over the same galaxies. The mean shift of each individual cluster $i$ is computed as

$$
\delta_{\mathrm{cm}, \mathrm{i}}=\frac{1}{2} \frac{\left\langle\beta_{\mathrm{C} 2015}\right\rangle-\left\langle\beta_{\mathrm{D} 2}\right\rangle}{\left\langle\beta_{\mathrm{D} 2}\right\rangle}
$$

with the variance, assuming a Gaussian of the same variance as a top-hat distribution between $\beta_{\mathrm{D} 2}$ and $\beta_{\mathrm{C} 2015}$, given by

$$
\sigma_{\mathrm{cm}, \mathrm{i}}=\frac{1}{\sqrt{3}}\left|\delta_{\mathrm{cm}, \mathrm{i}}\right|
$$

To derive the cosmic variance contribution $\sigma_{\mathrm{cv}}$ of each cluster we use a jackknife estimate over the four pointings of CFHTLS Deep:

$$
\sigma_{\mathrm{cv}, \mathrm{i}}=\sqrt{\frac{3}{4} \sum_{j=1}^{4}\left[\left(\left\langle\beta_{i}\right\rangle_{\neg j}-\left\langle\beta_{i}\right\rangle\right)^{2}\right] /\left\langle\beta_{i}\right\rangle},
$$

with $\left\langle\beta_{i}\right\rangle_{\neg j}$ being the lensing-weighted mean $\beta$ excluding CFHTLS Deep pointing $j$ and $\left\langle\beta_{i}\right\rangle$ the average over all pointings. The final shift of each individual cluster takes into account the lensing weight $w$ and is given by

$$
\delta_{\mathrm{cm}, \mathrm{i}}=w_{i} \delta_{\mathrm{cm}, \mathrm{i}}
$$

with variance

$$
\sigma_{\mathrm{cm}, \mathrm{i}}=w_{i} \sigma_{i}
$$

where $\sigma_{i}$ incorporates the contribution from cosmic variance and photo-z errors:

$$
\sigma_{i}=\sqrt{\sigma_{\mathrm{cv}, \mathrm{i}}^{2}+\sigma_{\mathrm{cm}, \mathrm{i}}^{2}} .
$$

In selecting our sources, we removed regions of colourmagnitude space that are contaminated by galaxies at the cluster redshift. Due to redshift uncertainties we estimate this to be at a level of about 2 per cent (see section 2.6). For this reason, we expect dilution of our source sample with cluster members to be minor. To account for residual cluster member dilution, we assume a value of $\delta_{\mathrm{cmd}}=0$ with an uncertainty of $\sigma_{\mathrm{cmd}}=0.02$.
The final shear calibration term is derived by

$$
S_{\mathrm{m}}=1-\delta_{\mathrm{cm}}-\delta_{\mathrm{sm}}-\delta_{\mathrm{cmd}}
$$

with the uncertainty given by

$$
\sigma_{\mathrm{S}}=\sqrt{\sigma_{\mathrm{cm}}^{2}+\sigma_{\mathrm{cv}}^{2}+\sigma_{\mathrm{sm}}^{2}+\sigma_{\mathrm{cmd}}^{2}} .
$$

We correct the measured lensing signal dividing by $S_{\mathrm{m}}$. Given the large statistical errors originating from shape noise the shear calibration error hardly carries weight. Nonetheless we take the uncertainty into account by remeasuring weak lensing masses with shear calibration values of $S_{\mathrm{m}} \pm \sigma_{\mathrm{S}}$ and adding the deviation from the actual best-fit value quadratically into our systematic error budget. The shear calibration values for the individual CODEX clusters can be seen in Table A1.

\section{A3 Surface density profile}

Our model for the $\Delta \Sigma$ profile of a cluster of given mass is not perfect: on small scales, the off-centring of redMaPPer-identified BCG candidates smears out the profile; on large scales, truncation reduces the surface density of the main halo, while correlated secondary haloes add to it; and there are additional differences between the mean density profiles of haloes and the NFW prescription, as measured from detailed $N$-body simulations. We calibrate these effects using simulated clusters of galaxies. To this end, we convert the shear maps extracted by Becker \& Kravtsov (2011) from the simulation labelled L1000W in Tinker et al. (2008). We use two snapshots with dark matter particles of mass $6.98 \times 10^{10} h^{-1} M_{\odot}$ in a box of comoving size $1 h^{-1} \mathrm{Gpc}$, one at $z_{d}=0.245$ for all haloes with $M_{200 \mathrm{c}} \geqslant 10^{14} h^{-1} M_{\odot}$ and one at $z_{d}=0.50$ for haloes with $M_{200 c} \geqslant 3 \times 10^{14} h^{-1} M_{\odot}$. We convert the gravitational shear maps centred on these haloes and simulated for sources at $z_{s}=1$ to observable reduced shear profiles. We run the mass likelihood described in subsection 2.10, using a covariance matrix including the mean shape noise of our cluster sample, LSS contributions calculated for the lensing-weighted stacked source $p(z)$ of our cluster sample, and intrinsic profile variations at the respective cluster mass and redshift. The surface density model differs from the one described in subsection 2.10 in that we use the massconcentration relation of Duffy et al. (2008) that better matches the cosmological parameters and resulting halo profiles of the L1000W simulation. Fig. A1 shows the mean recovered mass in bins of true mass without off-centring (in the two upper panels). We find no significant bias and no significant evolution of bias with cluster mass or redshift. The mean bias of all clusters in the two snapshots is $\Delta \log M_{200 \mathrm{c}}=0.001 \pm 0.002$. We include these corrections in our analysis and their uncertainty in our systematic error budget.

\section{A3.1 Concentration-mass relation}

We verify the robustness of the measured cluster masses with respect to the chosen concentration-mass relation. For that purpose we repeat the mass measurements on the simulated cluster sample, modifying the applied concentration-mass relation. In a first run we increase the original concentration value by 10 per cent, in a second run we increase it by 33 per cent with respect to the original value. The retrieved average logarithmic mass is lower by 0.002 in the first case and by 0.007 in the second case. If we further increase the concentration by 50 per cent with respect to the original relation we measure a logarithmic mass lower by 0.010 .

Cibirka et al. (2016) found a mean concentration value for the 
Table A1. Weak lensing shear calibration values from $\mathrm{p}(\mathrm{z})$ for all three CODEX subsamples of galaxy clusters. We set $\sigma_{\mathrm{cmd}}=\sigma_{\mathrm{sm}}=0.02$. The final shear and total uncertainty are given in equations $\mathrm{A} 7$, and $\mathrm{A} 8$, respectively.

\begin{tabular}{|c|c|c|c|c|c|c|}
\hline Subsample & CODEX ID & $\delta_{\mathrm{cm}}$ & $\sigma_{\mathrm{cm}}$ & $\sigma_{\mathrm{cv}}$ & $S_{\mathrm{m}}$ & $\sigma_{\mathrm{S}}$ \\
\hline \multirow[t]{36}{*}{ S-I } & 12451 & 0.023 & 0.013 & 0.005 & 0.977 & 0.032 \\
\hline & 13062 & 0.012 & 0.007 & 0.004 & 0.988 & 0.029 \\
\hline & 13390 & 0.025 & 0.014 & 0.005 & 0.975 & 0.032 \\
\hline & 16566 & 0.006 & 0.004 & 0.003 & 0.994 & 0.029 \\
\hline & 18127 & 0.014 & 0.008 & 0.004 & 0.986 & 0.030 \\
\hline & 24865 & 0.013 & 0.008 & 0.004 & 0.987 & 0.029 \\
\hline & 24872 & 0.006 & 0.004 & 0.003 & 0.994 & 0.029 \\
\hline & 24877 & 0.023 & 0.013 & 0.006 & 0.977 & 0.032 \\
\hline & 24981 & 0.004 & 0.003 & 0.003 & 0.996 & 0.029 \\
\hline & 25424 & 0.015 & 0.009 & 0.004 & 0.985 & 0.030 \\
\hline & 25953 & 0.013 & 0.008 & 0.003 & 0.987 & 0.030 \\
\hline & 27940 & 0.007 & 0.004 & 0.003 & 0.993 & 0.029 \\
\hline & 27974 & 0.011 & 0.007 & 0.003 & 0.989 & 0.029 \\
\hline & 29283 & 0.017 & 0.010 & 0.006 & 0.983 & 0.031 \\
\hline & 29284 & 0.017 & 0.010 & 0.005 & 0.983 & 0.030 \\
\hline & 29811 & 0.014 & 0.008 & 0.003 & 0.986 & 0.030 \\
\hline & 35361 & 0.007 & 0.004 & 0.003 & 0.993 & 0.029 \\
\hline & 35399 & 0.016 & 0.009 & 0.003 & 0.984 & 0.030 \\
\hline & 35646 & 0.010 & 0.006 & 0.003 & 0.990 & 0.029 \\
\hline & 36818 & 0.022 & 0.013 & 0.005 & 0.978 & 0.031 \\
\hline & 37098 & 0.017 & 0.010 & 0.005 & 0.983 & 0.030 \\
\hline & 41843 & 0.008 & 0.004 & 0.003 & 0.992 & 0.029 \\
\hline & 41911 & 0.007 & 0.004 & 0.003 & 0.993 & 0.029 \\
\hline & 43403 & 0.008 & 0.005 & 0.003 & 0.992 & 0.029 \\
\hline & 46649 & 0.024 & 0.014 & 0.005 & 0.976 & 0.032 \\
\hline & 47981 & 0.018 & 0.010 & 0.004 & 0.982 & 0.030 \\
\hline & 50492 & 0.016 & 0.009 & 0.004 & 0.984 & 0.030 \\
\hline & 50514 & 0.011 & 0.007 & 0.004 & 0.989 & 0.029 \\
\hline & 52480 & 0.020 & 0.012 & 0.006 & 0.980 & 0.031 \\
\hline & 53436 & 0.018 & 0.010 & 0.004 & 0.982 & 0.030 \\
\hline & 53495 & 0.011 & 0.006 & 0.003 & 0.989 & 0.029 \\
\hline & 54795 & 0.009 & 0.005 & 0.003 & 0.991 & 0.029 \\
\hline & 55181 & 0.017 & 0.010 & 0.005 & 0.983 & 0.030 \\
\hline & 56934 & 0.009 & 0.005 & 0.003 & 0.991 & 0.029 \\
\hline & 59915 & 0.011 & 0.007 & 0.003 & 0.989 & 0.029 \\
\hline & 64232 & 0.017 & 0.010 & 0.004 & 0.983 & 0.030 \\
\hline \multirow[t]{18}{*}{ S-II } & 13311 & 0.007 & 0.004 & 0.003 & 0.993 & 0.029 \\
\hline & 13315 & 0.024 & 0.014 & 0.006 & 0.976 & 0.032 \\
\hline & 13380 & -0.001 & 0.001 & 0.002 & 1.001 & 0.028 \\
\hline & 13390 & 0.025 & 0.014 & 0.005 & 0.975 & 0.032 \\
\hline & 13391 & -0.001 & 0.001 & 0.002 & 1.001 & 0.028 \\
\hline & 13400 & 0.013 & 0.007 & 0.003 & 0.987 & 0.029 \\
\hline & 17449 & 0.009 & 0.005 & 0.003 & 0.991 & 0.029 \\
\hline & 17453 & 0.002 & 0.001 & 0.003 & 0.998 & 0.028 \\
\hline & 54652 & 0.017 & 0.010 & 0.005 & 0.983 & 0.030 \\
\hline & 56934 & 0.009 & 0.005 & 0.003 & 0.991 & 0.029 \\
\hline & 57017 & 0.002 & 0.001 & 0.002 & 0.998 & 0.028 \\
\hline & 60076 & -0.002 & 0.001 & 0.002 & 1.002 & 0.028 \\
\hline & 60131 & 0.001 & 0.000 & 0.002 & 0.999 & 0.028 \\
\hline & 60155 & -0.001 & 0.001 & 0.003 & 1.001 & 0.028 \\
\hline & 64565 & 0.003 & 0.002 & 0.003 & 0.997 & 0.028 \\
\hline & 64636 & 0.008 & 0.004 & 0.003 & 0.992 & 0.029 \\
\hline & 210288 & -0.002 & 0.001 & 0.002 & 1.002 & 0.028 \\
\hline & 210306 & -0.002 & 0.001 & 0.002 & 1.002 & 0.028 \\
\hline \multirow[t]{4}{*}{ S-III } & 24925 & 0.002 & 0.001 & 0.003 & 0.998 & 0.028 \\
\hline & 27955 & 0.002 & 0.001 & 0.003 & 0.998 & 0.028 \\
\hline & 46647 & 0.001 & 0.001 & 0.002 & 0.999 & 0.028 \\
\hline & 54796 & -0.001 & 0.001 & 0.002 & 1.001 & 0.028 \\
\hline
\end{tabular}



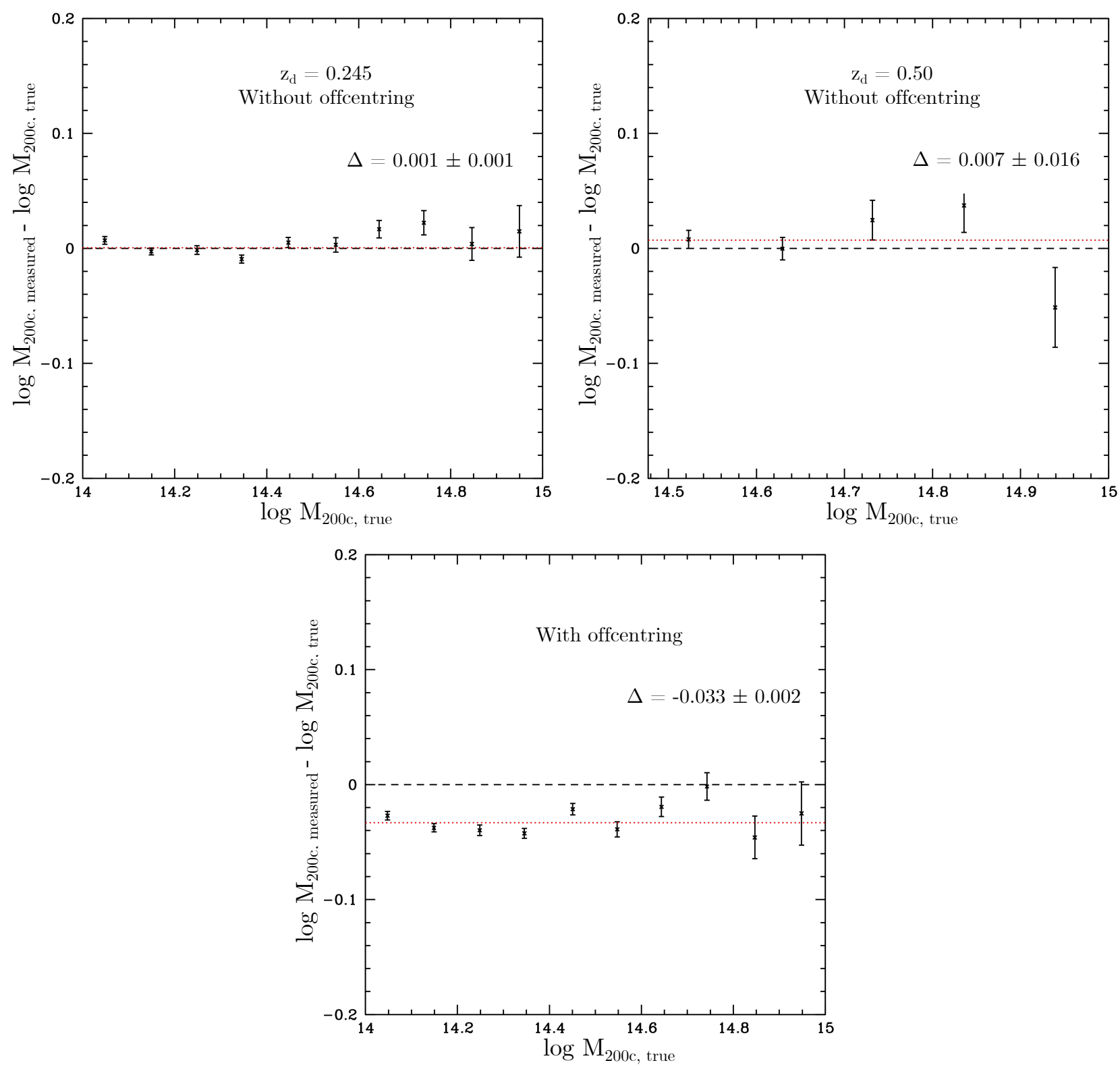

Figure A1. True mass vs. measured mass for perfectly well centred simulated galaxy clusters for the simulation at $z_{d}=0.245$ (upper left panel) and at $z_{d}=0.50$ (upper right panel). The true masses are recovered to a very good level in the weak lensing analysis. In the lower panel, the true mass vs. measured mass for the off-centred simulated galaxy clusters (all redshifts combined). The measured cluster masses are in average about 0.03 lower in logarithmic scale than the true masses.

stacked CODEX sample of $c=3.7_{-0.6}^{+0.7}$ which roughly corresponds to an uncertainty of 20 per cent. If we scale the concentration-mass relation by 20 per cent, once up and once down, we obtain logarithmic masses which are lower by 0.004 in the first case and higher by 0.002 in the second case. Taking these analyses into account we conclude that in the chosen range of between 500 and 2500 $h^{-1} \mathrm{kpc}$ the results are quite robust against modest modifications of the concentration-mass relation. Anyhow we will add the scatter $\left(\sigma_{\log M}=0.003\right)$ based on the 20 per cent modification into our systematic error budget.

\section{A3.2 Off-centring}

In our lensing analysis, we will define cluster centres as the most likely centre candidates identified by the redMaPPer algorithm. There are several failure modes of this assumption: sometimes it is not unambiguous from the photometric data which of the cluster galaxies is the central one, other times the true central galaxy might be lost to masking or, e.g. for ongoing mergers, there might not be a single central galaxy at all. These effects lead to a distribution of centre offsets that, on average, lower the cluster shear profiles on small scales. A lensing analysis could correct for this either by using an appropriately smoothed model (cf., e.g. Simet et al. 2016; Melchior et al. 2016, for redMaPPer lensing analyses using this approach) or by correcting for the average mass bias incurred in the 
fitting process to off-centred haloes. We do the latter, by shifting the centres of a fraction $\left(1-p_{\text {cen }}\right)$ of the simulated cluster fields by a (RA, dec) offset drawn from two independent Gaussians with a standard deviation of $340 \mathrm{~h}^{-1} \mathrm{kpc}$, the best-fit parameters of Cibirka et al. (2016). We find that the off-centring causes a mass bias of $\Delta \log M_{200 \mathrm{c}}=-0.033 \pm 0.002$ (see Fig. A1, lower panel), which we include in our analysis as a calibration factor. The dominant uncertainty in this offset does not result from the size of our simulated cluster sample, but from the width of the off-centring priors. From the constraints on off-centring derived in Rykoff et al. (2016) from $\mathrm{X}$-ray and SZ estimates of redMaPPer cluster centres, we approximate the uncertainty on the effect of off-centring as 50 per-cent of the fiducial amplitude, i.e. a systematic bias and uncertainty on mass of $\Delta \log M_{200 c}=-0.033$ and $\sigma_{\log M_{200 c}}=0.017$.

\section{A4 Systematic error budget}

We summarize our budget of systematic errors here. The following effects contribute to systematic uncertainty of our weak lensing cluster mass measurements:

- multiplicative error in shape measurements

- multiplicative error in our photometric estimate of $\beta$

- dilution of the source sample with cluster members

- mismatch of the fitted density profile to the truth

- uncertainty of mass-concentration relation

- uncertain prior on off-centring

Contributions from the effects named above are described in detail in the previous sections. An overview is given in Table A2. Multiplicative uncertainties in the measured profile amplitude are scaled up by $4 / 3$ (Melchior et al. 2016, their eqn. 53) to yield multiplicative errors in mass. The sign of $\Delta \log \Delta \Sigma$ and $\Delta \log M_{200 c}$ is defined such that if $<0$, the respective effect lowers the reconstructed $\Delta \Sigma$ and mass. We correct for the mean value of these biases in all massed we quote: the $\Delta \Sigma$ profiles we analyse with our likelihoods are corrected to account for the estimated mean value of the bias in $\beta$, and the recovered masses are re-scaled to correct for the biases expected from model profile mismatch and off-centring. For each cluster, we calculate a systematic uncertainty in mass as the squared sum of all above effects, where only the $p(z)$ bias differs from cluster to cluster.

\section{APPENDIX B: FIELDS WITH INCOMPLETE COLOUR INFORMATION}

The CODEX survey was planned to be covered by five-filter observations (ugriz), but in several occasions these observations have remained incomplete yet, resulting in coverage of some clusters only in four or fewer bands. Nonetheless, the colour-magnitude decision tree enables us to use even those pointings with incomplete colour information. However, a reduced accuracy of the measurements cannot be ruled out, which would lead to a scatter weakening the constraints or even inducing a bias on the mass estimates. We therefore have a closer look at those 31 pointings with complete coverage in five bands and recalculate $\beta$ leaving out certain filter information. This step artificially creates full samples with incomplete colour information. In detail we analyze the following filter combinations: griz, ugri, and uriz (see Fig. C1).

In more detail, first we estimate the geometrical distance ratio $\beta_{\text {ugriz }}$ (cf. equation 4) using the complete five-filter ugriz colour information and measure the galaxy cluster mass. This gives us a baseline mass estimate for our galaxy cluster sample. We repeat this mass estimate for each single cluster based on $\beta_{\text {griz }}, \beta_{\text {ugri }}$ and $\beta_{\text {uriz }}$ and compare those masses to the five-band mass values. We do this by applying linear regression, weighting each cluster with the corresponding mass uncertainties $\left(w=1 /\left(\sigma_{m 1}^{2}+\sigma_{m 2}^{2}\right)\right)$. The uncertainties on the fit parameters are then calculated from jackknife estimates.

Given the large uncertainties in the individual masses, the uncertainties for the fit parameters are quite large as well. However, we do not detect a significant multiplicative bias within the uncertainties.

\section{B1 Richness from three-band photometry}

Cluster richness is commonly estimated from four-band griz photometric data. For a small subset of our clusters ( 3 with ugri, 1 with uriz), we do not have this full information available. In this section, we investigate whether richnesses estimated from three-band information only ( $\mathrm{gri}$ or $\mathrm{riz}$ ) show considerable deviations from the fiducial griz case.

To this end, we run redMaPPer on gri and riz data only, for all clusters with griz observations from our pointed CODEX followup data. Comparison of these and the fiducial griz richnesses are shown in (Fig. C2). We fit the newly obtained richness estimates based on incomplete colour information with respect to the original values applying a linear regression and find that lack of $g$ or $z$-band does not change the richness estimates significantly. The best-fit relations of CFHT-griz vs CFHT-gri and CFHT-griz vs CFHT-riz richnesses are approximated by $\lambda_{\text {gri }}=(1.00 \pm 0.10) \lambda_{\text {griz }}+(3 \pm 9)$, and $\lambda_{\text {riz }}=(1.02 \pm 0.07) \lambda_{\text {griz }}+(5 \pm 6)$, respectively. The bestfit relations show the CFHT-griz richness is more consistent with CFHT-riz richness, as a result we use this richness.

The original plan was to incorporate all 36 clusters from sample S-I into the Bayesian analysis, described in section 4, but since the calibration is too uncertain for the $<5$ band clusters, and the sample is too small, we decided to only include the 5 band filter clusters in the final analysis.

\section{APPENDIX C: EXCLUDED WEAK LENSING SAMPLES}

From the initial sample of 407 clusters, and our follow-up observations with different redshift and richness range than the initial sample, we define three lensing samples with distinct selection functions, that are not part of the analysis, due to lacking weak lensing mass information, CFHT richness information, or richness incompleteness. Even though we do not use these clusters to calibrate the richness-mass relation, their weak lensing mass measurements are robust, so we present their mass measurements, along with sky coordinates, X-ray luminosities, spectroscopic and optical redshifts, and SDSS richness.

- The definition of the first lensing sample of 36 clusters S-I is given in section 2.1. The S-I lensing sample is listed in two separate places, in Table 1 (cleaned lensing sample of 25 clusters that went into the richness-mass analysis) and Table C1 (11 excluded clusters from the analysis).

- Our second lensing sample of 18 clusters, hereafter S-II, is selected only by their ROSAT excess. Its position is required to fall inside the CFHTLS footprint, but we do not require a rich optical counterpart in the CFHT observations to be present. Therefore, all clusters in S-II have "-" listed in the CFHT richness and CFHT 
Table A2. Systematic uncertainties in weak lensing mass likelihood.

\begin{tabular}{ccccccc}
\hline \hline Effect & $\Delta \log \Delta \Sigma$ & $\sigma_{\log \Delta \Sigma}$ & $\Delta \log M_{200 c}$ & $\sigma_{\log M_{200 c}}$ & Section/Appendix & Notes \\
\hline \hline Shear bias & 0.000 & 0.009 & 0.000 & 0.011 & A1 & Assuming 2\% \\
$p(z)$ bias & - & - & - & - & 2.6 & See Table A3 \\
Cluster member dilution & 0.000 & 0.009 & 0.000 & 0.011 & 2.6 & Assuming 2\% \\
\hline Model profile mismatch & - & - & 0.001 & 0.002 & A3 & - \\
$C(M)$ & - & - & 0.000 & 0.003 & 2.9 & - \\
Off-centring & - & - & -0.033 & 0.017 & $\mathrm{~A} 3.2$ & - \\
\hline \hline
\end{tabular}

redshift columns in Appendix, Table C2. For the purpose of feasible lensing analysis, we also require that $z_{\mathrm{RM}}$,SDSS $>0.1$. We note that clusters 13390 and 56934 are in both S-I and S-II, but do not have CFHT richness estimates. Unlike S-I and S-III, which are our dedicated observations, S-II shapes are from the public CFHTLenS catalogs (Heymans et al. 2012), which was before the introduction of self-calibration version of the lensfit.

- Our third lensing sample of 4 clusters, hereafter S-III, follow the same treatment as S-I, i.e., they are processed with the self-calibrating version of the lensfit, as described in section 2.5, following the calibration of Fenech Conti et al. (2017). Initially, these clusters fulfilled the selection criteria of the primary sample, but were later revised to $z_{\mathrm{RM} \text {,SDSS }}<0.35$.

The positions in the sky of clusters in each of these samples is shown in Figure C3.

\section{C1 Imaging data for S-II}

In addition to S-I and S-III pointings, for S-II, we make use of the publicly available imaging data from the CFHT Lensing Survey $^{8}$ (CFHTLenS, see Erben et al. 2013; Heymans et al. 2012) to include those CODEX clusters falling into the CFHTLS footprint and download the available reduced imaging data for 87 additional CFHT pointings. A summary of the all used images can be seen in Appendix D. The data reduction steps, detailed in section 2.2, for S-II remain the same as in S-I, and S-III.

For the cluster sample S-II falling into the footprint of the CFHTLS we create the corresponding multiband-photometry catalogues in the same way as described in section 2.3.

For the shape measurement, in the case of the cluster sample S-II we make use of the publicly available lensfit shape measurement data of CFHTLenS (see Miller et al. 2013) and download the corresponding catalogues from their website. In contrast to the lensfit version used in this work the version used in the CFHTLenS data release was not self-calibrating yet. We therefore include the following correction terms to the measured ellipticities (see Eqns. 17 and 19 in Heymans et al. 2012), a multiplicative one:

$$
m\left(v_{\mathrm{SN}}, r\right)=\frac{B}{\log \left(v_{\mathrm{SN}}\right)} \exp ^{-r A v_{\mathrm{SN}}}
$$

with $A=0.057$ and $B=-0.37$, which has to be applied through a weighted ensemble average correction, rather than dividing by $(1+m)$ on a galaxy-by-galaxy basis, and an additive one:

$$
c_{2}=\operatorname{Max}\left[\frac{F \log _{10}\left(v_{\mathrm{SN}}\right)-G}{1+\left(\frac{\mathrm{r}}{\mathrm{r}_{0}}\right)^{H}}, 0\right],
$$

with $F=11.910, G=12.715, H=2.458, r_{0}=0.01^{\prime}$, which has to be added only to $e_{2}$.

Since our data likelihood function, in section 4 , assumes that all the clusters in the analysis have both SDSS and CFHT richness estimates, this excludes the entire sample S-II and two clusters (CODEX ID 46647 and 54796) from S-III. For the last two clusters in S-III (CODEX ID 24925 and 27955), with redshifts $z \sim 0.3$, we did not include them in the analysis, as they fell outside of our original redshift region of $0.35<z<0.65$.

\footnotetext{
8 http://cfhtlens.org
} 
Table A3. Systematic uncertainties in weak lensing mass likelihood for individual clusters for all three subsamples.

Left column shows lower and upper uncertainty interval combined from $\mathrm{p}(\mathrm{z})$, shear bias and cluster member dilution, right column shows total systematic error budget.

\begin{tabular}{|c|c|c|c|}
\hline Subsample & CODEX ID & $\sigma_{\ln M_{200 c}, \sigma_{\mathrm{S}}, \text { syst }}$ & $\sigma_{\ln } \boldsymbol{M}_{200 c}$, total,syst \\
\hline \multirow[t]{36}{*}{ S-I } & 12451 & 0.021 & 0.027 \\
\hline & 13062 & - & - \\
\hline & 13390 & 0.021 & 0.027 \\
\hline & 16566 & 0.015 & 0.023 \\
\hline & 18127 & 0.027 & 0.032 \\
\hline & 24865 & 0.021 & 0.027 \\
\hline & 24872 & 0.017 & 0.024 \\
\hline & 24877 & 0.023 & 0.029 \\
\hline & 24981 & 0.017 & 0.024 \\
\hline & 25424 & 0.017 & 0.024 \\
\hline & 25953 & 0.019 & 0.026 \\
\hline & 27940 & 0.019 & 0.026 \\
\hline & 27974 & 0.019 & 0.026 \\
\hline & 29283 & 0.019 & 0.026 \\
\hline & 29284 & 0.019 & 0.026 \\
\hline & 29811 & 0.023 & 0.029 \\
\hline & 35361 & 0.023 & 0.029 \\
\hline & 35399 & 0.019 & 0.026 \\
\hline & 35646 & 0.015 & 0.023 \\
\hline & 36818 & 0.019 & 0.026 \\
\hline & 37098 & - & - \\
\hline & 41843 & 0.017 & 0.024 \\
\hline & 41911 & 0.019 & 0.026 \\
\hline & 43403 & 0.019 & 0.026 \\
\hline & 46649 & 0.021 & 0.027 \\
\hline & 47981 & 0.017 & 0.024 \\
\hline & 50492 & 0.023 & 0.029 \\
\hline & 50514 & 0.021 & 0.027 \\
\hline & 52480 & 0.017 & 0.024 \\
\hline & 53436 & - & - \\
\hline & 53495 & - & - \\
\hline & 54795 & 0.019 & 0.026 \\
\hline & 55181 & 0.021 & 0.027 \\
\hline & 56934 & 0.015 & 0.023 \\
\hline & 59915 & 0.021 & 0.027 \\
\hline & 64232 & 0.017 & 0.024 \\
\hline \multirow[t]{18}{*}{ S-II } & 13311 & 0.019 & 0.026 \\
\hline & 13315 & 0.021 & 0.027 \\
\hline & 13380 & 0.017 & 0.024 \\
\hline & 13390 & 0.021 & 0.027 \\
\hline & 13391 & 0.019 & 0.026 \\
\hline & 13400 & 0.021 & 0.027 \\
\hline & 17449 & 0.017 & 0.024 \\
\hline & 17453 & 0.017 & 0.024 \\
\hline & 54652 & 0.019 & 0.026 \\
\hline & 56934 & 0.015 & 0.023 \\
\hline & 57017 & 0.017 & 0.024 \\
\hline & 60076 & 0.017 & 0.024 \\
\hline & 60131 & 0.019 & 0.026 \\
\hline & 60155 & 0.015 & 0.023 \\
\hline & 64565 & 0.017 & 0.024 \\
\hline & 64636 & - & - \\
\hline & 210288 & 0.015 & 0.023 \\
\hline & 210306 & 0.015 & 0.023 \\
\hline \multirow[t]{4}{*}{ S-III } & 24925 & 0.019 & 0.026 \\
\hline & 27955 & 0.017 & 0.024 \\
\hline & 46647 & 0.015 & 0.023 \\
\hline & 54796 & 0.019 & 0.026 \\
\hline
\end{tabular}



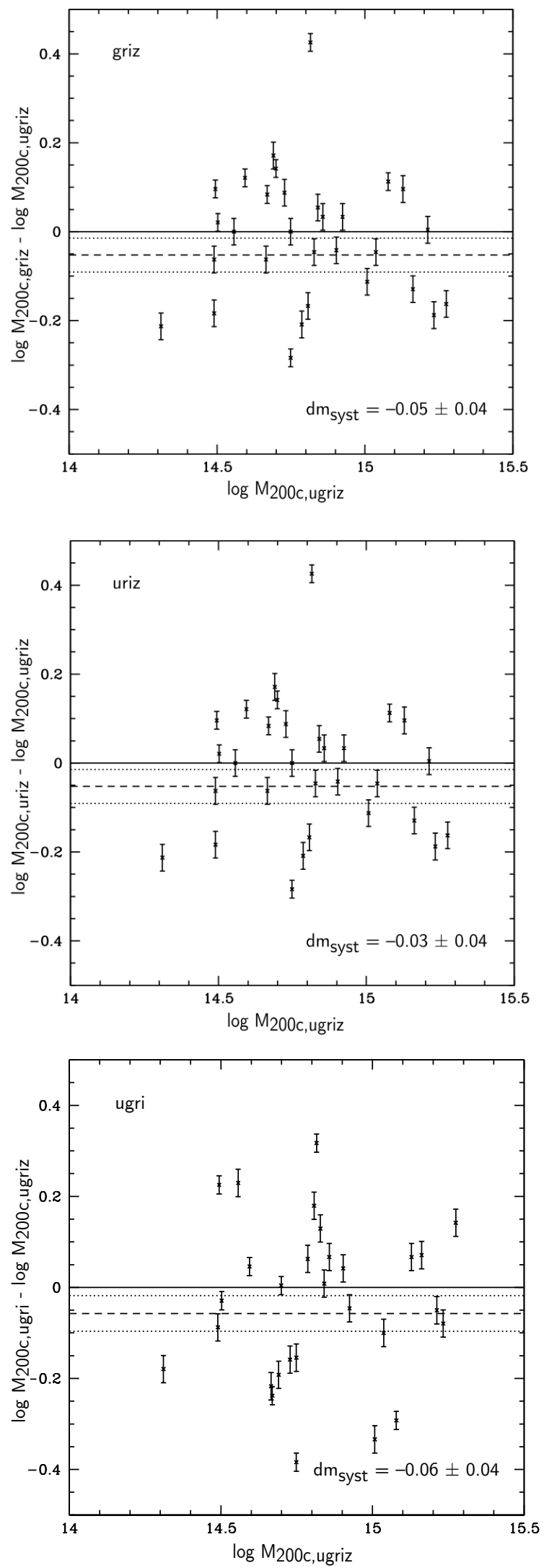

Figure C1. Weak lensing mass estimates based on ugriz photometry vs. four-filter photometry in griz (upper left panel), uriz, (upper right panel), and ugri (lower panel). The errorbars only show the systematic error in mass measurement since the by far larger statistical error should not significantly depend on the available filter combination. We correct our mass estimates based on griz, uriz, and ugri photometry and include the uncertainty into our systematic error estimate. 
Table C1. Lensing sample S-I clusters excluded from the richness-mass analysis.

\begin{tabular}{|c|c|}
\hline 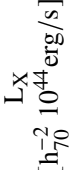 & 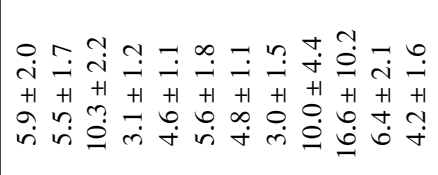 \\
\hline$\sum_{\substack{\infty \\
0}}^{\infty} \sum_{\substack{\infty \\
\Sigma}}^{+}$ & 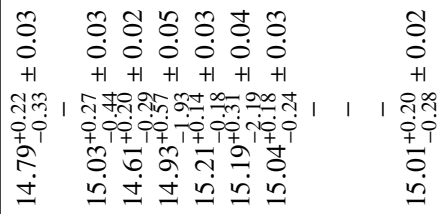 \\
\hline 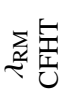 & 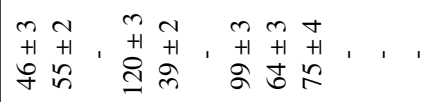 \\
\hline 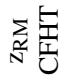 & 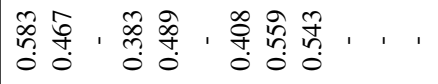 \\
\hline$\sum_{\substack{n \\
n}}^{n}$ & 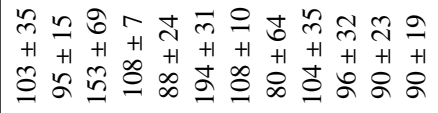 \\
\hline$\sum_{\text {N }}^{n}$ & 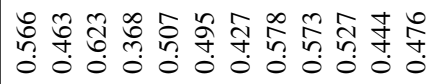 \\
\hline N & 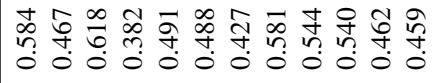 \\
\hline 善壱 & 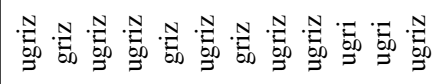 \\
\hline 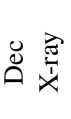 & 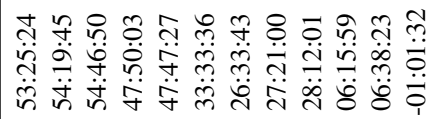 \\
\hline & 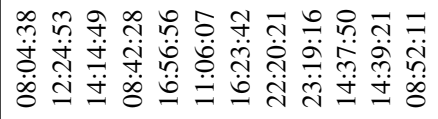 \\
\hline$\stackrel{\text { ڤ̆ }}{0}$ & 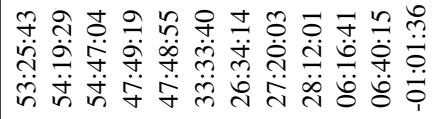 \\
\hline & 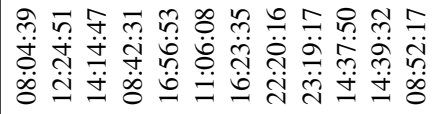 \\
\hline $\begin{array}{l}n \\
\frac{1}{11} \\
\text { 空 }\end{array}$ & 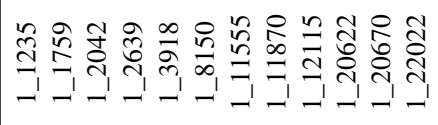 \\
\hline 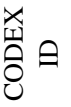 & 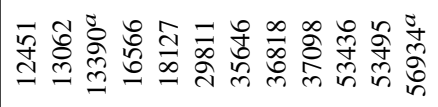 \\
\hline
\end{tabular}


K. Kiiveri et al.
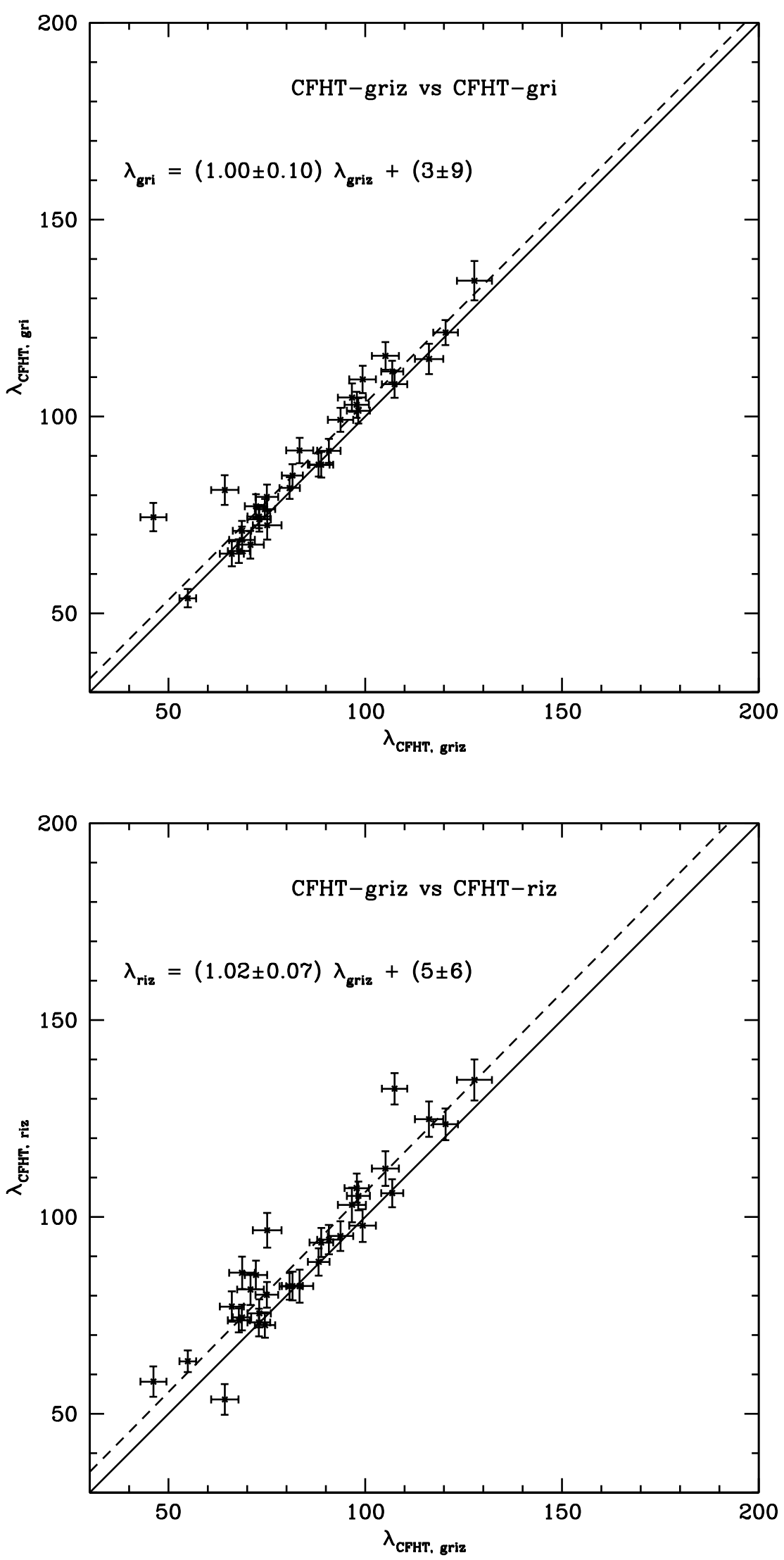

Figure C2. Richness estimated from CFHT imaging, griz vs. gri and griz vs. riz for all clusters covered in griz. 


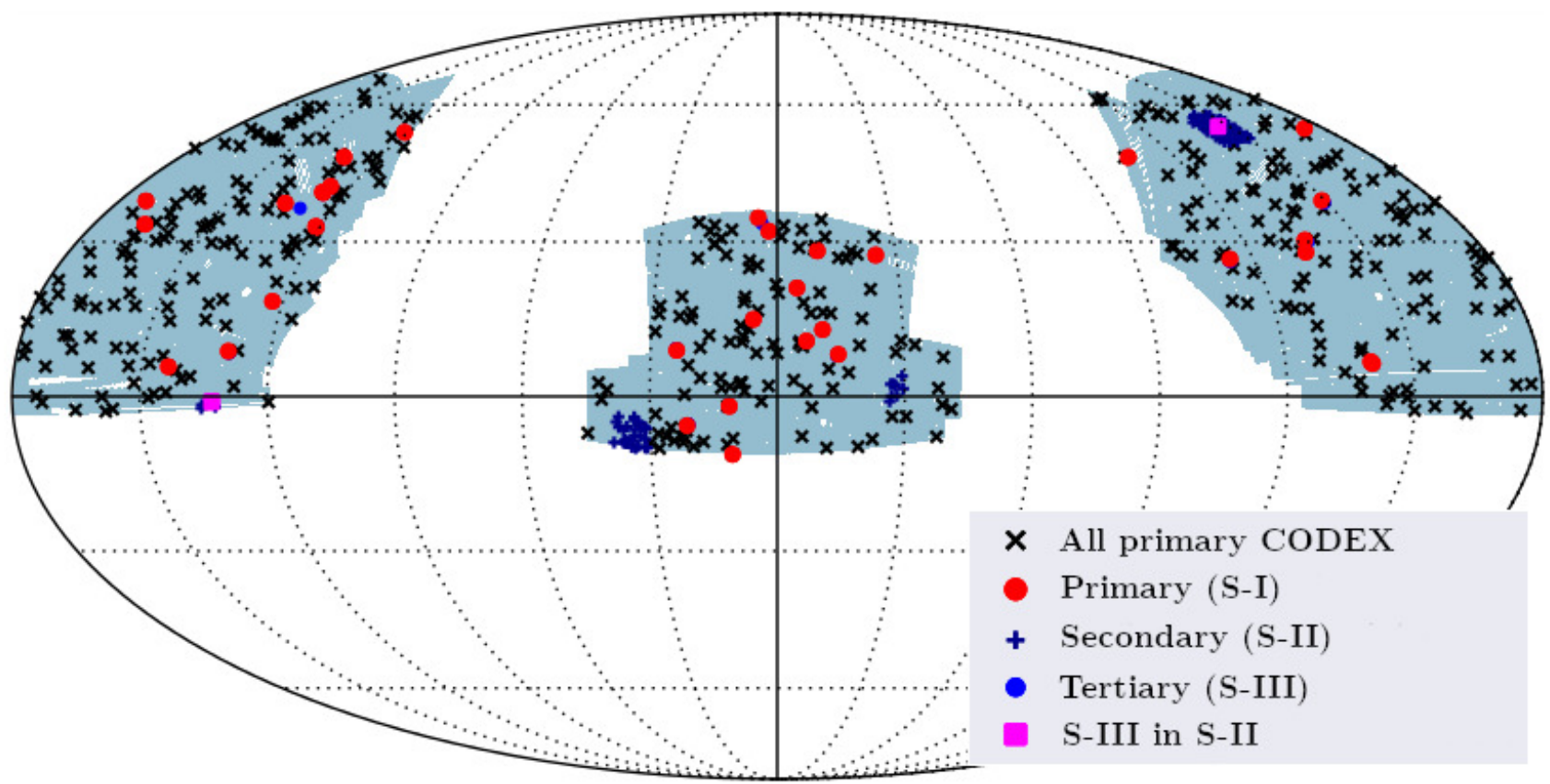

Figure C3. Positions of CODEX clusters in the sky. SDSS footprint is shown as grey area. Black crosses correspond to initial CODEX sample of 407 clusters, red points to CODEX lensing sample of 36 clusters (S-I) and dark blue crosses to the secondary sample, (S-II). Additionally, the blue points correspond to tertiary sample (S-III). Most of the tertiary targets (S-III) are secondary objects in primary target pointings and thus overplotted in pink. 
Table C2. Weak lensing mass catalogue for the sample S-II (covered by CFHTLS Wide, see Erben et al. 2013), and S-III $(z<0.35)$.

\begin{tabular}{|c|c|c|}
\hline 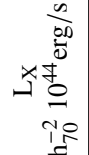 & 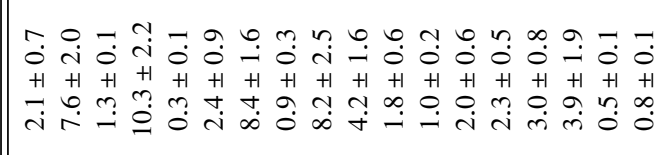 & 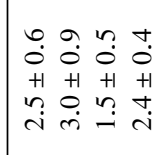 \\
\hline$\sum_{\substack{\infty \\
0}}^{\infty}$ & 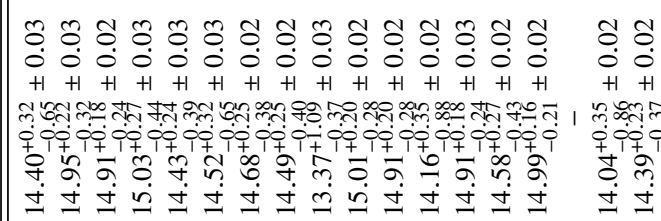 & 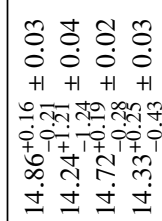 \\
\hline & 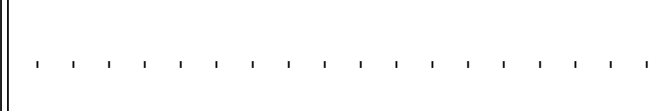 & 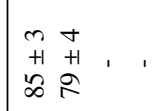 \\
\hline & $1,1,1,1,1,1,1,1,1$ & 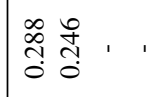 \\
\hline & 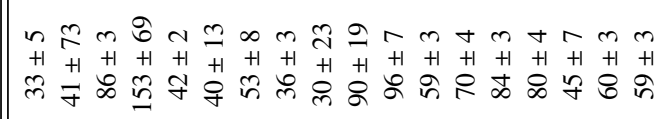 & 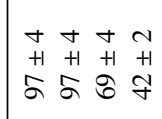 \\
\hline 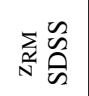 & 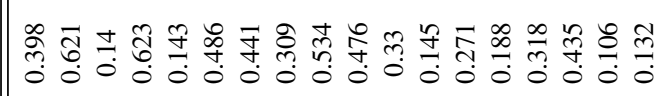 & 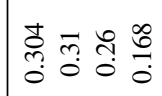 \\
\hline & 吕 & 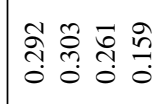 \\
\hline & 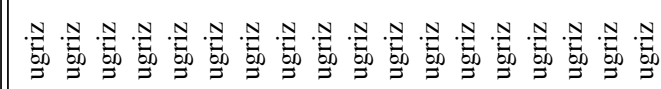 & 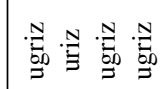 \\
\hline & 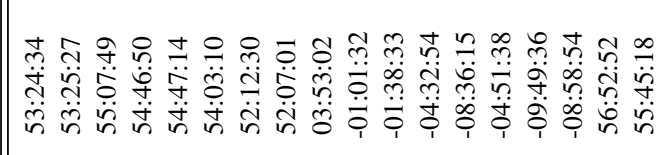 & 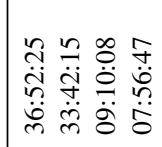 \\
\hline & 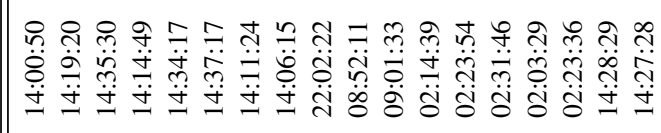 & 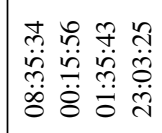 \\
\hline & 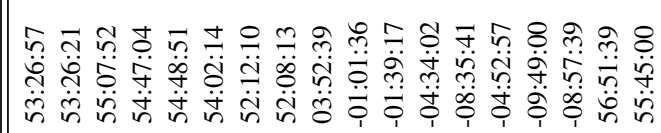 & 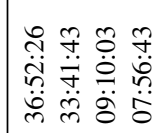 \\
\hline & 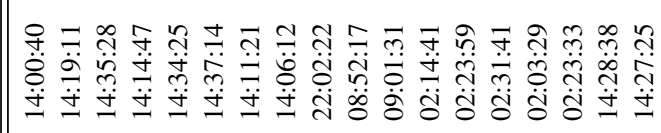 & 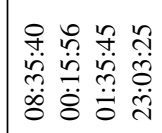 \\
\hline 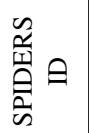 & 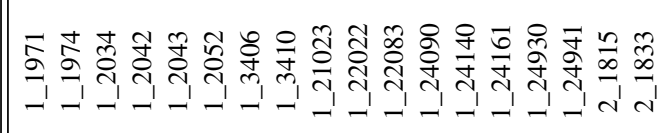 & 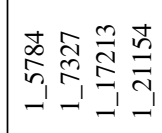 \\
\hline 岸 & 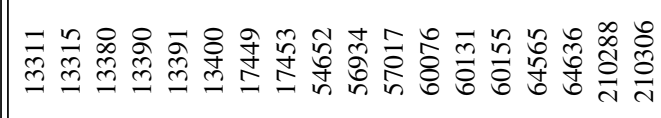 & 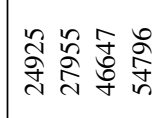 \\
\hline & 咅 & 素 \\
\hline
\end{tabular}


APPENDIX D: OBSERVATIONAL PROPERTIES OF THE CODEX IMAGING DATA

Table D1: CODEX cluster sample S-I $\left(\lambda_{\mathrm{RM}, \text { SDSS }}>60\right.$ and $\left.0.35<z<0.65\right)$, targeted fields only

\begin{tabular}{|c|c|c|c|c|c|}
\hline$\overline{\overline{\text { CODEX ID }}}$ & Filter & N. of exp. & $\begin{array}{c}\text { expos. time } \\
{[\mathrm{s}]}\end{array}$ & $\begin{array}{c}m_{\lim } \\
{[\mathrm{AB} \text { mag] }}\end{array}$ & $\begin{array}{c}\text { Seeing } \\
{\left[{ }^{\prime \prime}\right]}\end{array}$ \\
\hline 12451 & u.MP9301 & 5 & 2601 & 25.20 & 0.83 \\
\hline 12451 & g.MP9401 & 5 & 1051 & 25.12 & 1.08 \\
\hline 12451 & r.MP9601 & 9 & 2492 & 25.01 & 0.94 \\
\hline 12451 & i.MP9702 & 15 & 8403 & 24.89 & 0.61 \\
\hline 12451 & z.MP9801 & 2 & 1081 & 22.56 & 1.04 \\
\hline 13062 & g.MP9401 & 7 & 1471 & 25.34 & 0.82 \\
\hline 13062 & r.MP9601 & 6 & 1261 & 24.65 & 0.77 \\
\hline 13062 & i.MP9702 & 3 & 1681 & 24.40 & 0.98 \\
\hline 13062 & z.MP9801 & 4 & 2321 & 23.41 & 0.75 \\
\hline 16566 & u.MP9301 & 8 & 4482 & 24.92 & 1.15 \\
\hline 16566 & g.MP9401 & 3 & 1021 & 24.43 & 0.99 \\
\hline 16566 & r.MP9601 & 6 & 3001 & 24.86 & 1.17 \\
\hline 16566 & i.MP9702 & 8 & 4482 & 24.80 & 0.71 \\
\hline 16566 & z.MP9801 & 8 & 4482 & 23.57 & 0.73 \\
\hline 18127 & g.MP9401 & 3 & 6301 & 24.71 & 0.82 \\
\hline 18127 & r.MP9601 & 5 & 1291 & 24.48 & 0.74 \\
\hline 18127 & i.MP9702 & 8 & 4482 & 24.60 & 0.54 \\
\hline 18127 & z.MP9801 & 5 & 2701 & 23.34 & 0.78 \\
\hline 24865 & u.MP9301 & 5 & 2601 & 25.24 & 0.79 \\
\hline 24865 & g.MP9401 & 6 & 1261 & 25.16 & 0.91 \\
\hline 24865 & r.MP9601 & 5 & 1351 & 24.63 & 0.75 \\
\hline 24865 & i.MP9702 & 7 & 3921 & 24.71 & 0.73 \\
\hline 24865 & z.MP9801 & 3 & 1621 & 23.12 & 0.68 \\
\hline 24872 & u.MP9301 & 5 & 2801 & 25.32 & 1.05 \\
\hline 24872 & g.MP9401 & 3 & 1021 & 25.13 & 0.97 \\
\hline 24872 & r.MP9601 & 3 & 1494 & 24.81 & 0.87 \\
\hline 24872 & i.MP9702 & 8 & 4482 & 24.77 & 0.84 \\
\hline 24872 & z.MP9801 & 4 & 2241 & 23.60 & 0.88 \\
\hline 24877 & u.MP9301 & 5 & 2801 & 25.32 & 1.05 \\
\hline 24877 & g.MP9401 & 3 & 1021 & 25.13 & 0.97 \\
\hline 24877 & r.MP9601 & 3 & 1494 & 24.81 & 0.87 \\
\hline 24877 & i.MP9702 & 8 & 4482 & 24.77 & 0.84 \\
\hline 24877 & z.MP9801 & 4 & 2241 & 23.60 & 0.88 \\
\hline 24981 & u.MP9301 & 5 & 2601 & 25.30 & 0.93 \\
\hline 24981 & g.MP9401 & 5 & 1601 & 25.33 & 0.65 \\
\hline 24981 & r.MP9601 & 12 & 7406 & 25.49 & 0.64 \\
\hline 24981 & i.MP9701 & 4 & 961 & 23.80 & 0.89 \\
\hline 24981 & i.MP9702 & 6 & 3361 & 24.59 & 0.60 \\
\hline 24981 & z.MP9801 & 4 & 1441 & 22.91 & 0.81 \\
\hline 25424 & u.MP9301 & 15 & 7803 & 25.73 & 1.03 \\
\hline 25424 & g.MP9401 & 4 & 841 & 24.99 & 0.76 \\
\hline 25424 & r.MP9601 & 5 & 1351 & 24.51 & 0.65 \\
\hline 25424 & i.MP9702 & 8 & 4482 & 24.73 & 0.65 \\
\hline 25424 & z.MP9801 & 4 & 2161 & 23.27 & 0.46 \\
\hline 25953 & u.MP9301 & 4 & 2081 & 25.09 & 0.85 \\
\hline 25953 & g.MP9401 & 5 & 1051 & 25.21 & 0.91 \\
\hline 25953 & r.MP9601 & 4 & 991 & 24.58 & 0.84 \\
\hline 25953 & i.MP9702 & 8 & 4482 & 24.92 & 0.72 \\
\hline 25953 & z.MP9801 & 4 & 2161 & 23.62 & 0.83 \\
\hline
\end{tabular}




\begin{tabular}{|c|c|c|c|c|c|}
\hline CODEX ID & Filter & N. of exp. & $\begin{array}{c}\text { expos. time } \\
{[\mathrm{s}]}\end{array}$ & $\begin{array}{c}m_{\lim } \\
{[\mathrm{AB} \text { mag] }}\end{array}$ & $\begin{array}{c}\text { Seeing } \\
{\left[{ }^{\prime \prime}\right]}\end{array}$ \\
\hline 27940 & u.MP9301 & 5 & 2861 & 25.21 & 0.81 \\
\hline 27940 & g.MP9401 & 3 & 1080 & 25.04 & 0.68 \\
\hline 27940 & r.MP9601 & 3 & 1500 & 24.33 & 0.57 \\
\hline 27940 & i.MP9702 & 9 & 5042 & 24.77 & 0.91 \\
\hline 27940 & z.MP9801 & 4 & 2373 & 23.36 & 0.86 \\
\hline 27974 & u.MP9301 & 4 & 2289 & 25.23 & 0.82 \\
\hline 27974 & g.MP9401 & 3 & 1080 & 25.01 & 0.65 \\
\hline 27974 & r.MP9601 & 3 & 1500 & 24.31 & 0.67 \\
\hline 27974 & i.MP9702 & 8 & 4481 & 24.65 & 0.44 \\
\hline 27974 & z.MP9801 & 4 & 2373 & 23.29 & 0.86 \\
\hline 29283 & u.MP9301 & 5 & 2601 & 25.22 & 0.90 \\
\hline 29283 & g.MP9401 & 6 & 1261 & 25.23 & 1.20 \\
\hline 29283 & r.MP9601 & 3 & 930 & 24.45 & 0.76 \\
\hline 29283 & i.MP9702 & 8 & 4482 & 24.84 & 0.56 \\
\hline 29283 & z.MP9801 & 4 & 2161 & 23.24 & 0.87 \\
\hline 29284 & u.MP9301 & 5 & 2601 & 25.22 & 0.90 \\
\hline 29284 & g.MP9401 & 6 & 1261 & 25.23 & 1.20 \\
\hline 29284 & r.MP9601 & 3 & 930 & 24.45 & 0.76 \\
\hline 29284 & i.MP9702 & 8 & 4482 & 24.84 & 0.56 \\
\hline 29284 & z.MP9801 & 4 & 2161 & 23.24 & 0.87 \\
\hline 29811 & u.MP9301 & 4 & 2081 & 24.85 & 0.84 \\
\hline 29811 & g.MP9401 & 10 & 2102 & 25.45 & 0.93 \\
\hline 29811 & r.MP9601 & 8 & 1981 & 24.86 & 0.78 \\
\hline 29811 & i.MP9702 & 6 & 3361 & 24.51 & 0.44 \\
\hline 29811 & z.MP9801 & 4 & 2161 & 23.26 & 0.51 \\
\hline 35361 & u.MP9301 & 10 & 5202 & 25.49 & 0.77 \\
\hline 35361 & g.MP9401 & 5 & 1051 & 25.06 & 0.96 \\
\hline 35361 & r.MP9601 & 6 & 1561 & 24.63 & 0.94 \\
\hline 35361 & i.MP9702 & 7 & 3921 & 24.68 & 0.57 \\
\hline 35361 & z.MP9801 & 4 & 2321 & 23.14 & 0.83 \\
\hline 35399 & u.MP9301 & 7 & 3641 & 24.94 & 0.94 \\
\hline 35399 & g.MP9401 & 3 & 901 & 24.81 & 0.62 \\
\hline 35399 & r.MP9601 & 3 & 1380 & 24.39 & 0.67 \\
\hline 35399 & i.MP9702 & 8 & 4481 & 24.58 & 0.55 \\
\hline 35399 & z.MP9801 & 4 & 2161 & 23.35 & 0.88 \\
\hline 35646 & g.MP9401 & 5 & 1601 & 25.44 & 0.72 \\
\hline 35646 & r.MP9601 & 12 & 7178 & 25.42 & 0.58 \\
\hline 35646 & i.MP9701 & 5 & 1201 & 24.08 & 0.60 \\
\hline 35646 & z.MP9801 & 7 & 2521 & 23.51 & 0.97 \\
\hline 36818 & u.MP9301 & 5 & 2861 & 25.29 & 1.07 \\
\hline 36818 & g.MP9401 & 3 & 1020 & 25.03 & 0.82 \\
\hline 36818 & r.MP9601 & 3 & 1501 & 24.48 & 0.88 \\
\hline 36818 & i.MP9702 & 10 & 5602 & 24.73 & 0.45 \\
\hline 36818 & z.MP9801 & 10 & 5884 & 23.69 & 0.63 \\
\hline 37098 & u.MP9301 & 5 & 2861 & 25.25 & 0.85 \\
\hline 37098 & g.MP9401 & 3 & 1020 & 25.10 & 0.65 \\
\hline 37098 & r.MP9601 & 3 & 1500 & 24.69 & 0.60 \\
\hline 37098 & i.MP9702 & 8 & 4481 & 24.66 & 0.70 \\
\hline 37098 & z.MP9801 & 4 & 2373 & 23.38 & 0.49 \\
\hline 41843 & u.MP9301 & 5 & 2801 & 25.30 & 0.83 \\
\hline 41843 & g.MP9401 & 3 & 1020 & 25.12 & 0.71 \\
\hline 41843 & r.MP9601 & 6 & 2989 & 24.98 & 0.67 \\
\hline 41843 & i.MP9702 & 8 & 4481 & 24.90 & 0.73 \\
\hline 41843 & z.MP9801 & 8 & 4481 & 23.52 & 0.67 \\
\hline
\end{tabular}




\begin{tabular}{|c|c|c|c|c|c|}
\hline CODEX ID & Filter & N. of exp. & $\begin{array}{c}\text { expos. time } \\
{[\mathrm{s}]}\end{array}$ & $\begin{array}{c}m_{\lim } \\
{[\mathrm{AB} \text { mag] }}\end{array}$ & $\begin{array}{c}\text { Seeing } \\
{\left[{ }^{\prime \prime}\right]}\end{array}$ \\
\hline 41911 & u.MP9301 & 6 & 3361 & 25.37 & 0.86 \\
\hline 41911 & g.MP9401 & 3 & 1020 & 25.10 & 0.91 \\
\hline 41911 & r.MP9601 & 3 & 1494 & 24.49 & 0.99 \\
\hline 41911 & i.MP9702 & 8 & 4481 & 24.74 & 0.65 \\
\hline 41911 & z.MP9801 & 4 & 2241 & 23.33 & 0.64 \\
\hline 43403 & u.MP9301 & 5 & 2601 & 25.22 & 0.84 \\
\hline 43403 & g.MP9401 & 5 & 1071 & 25.06 & 0.87 \\
\hline 43403 & r.MP9601 & 3 & 630 & 24.25 & 0.87 \\
\hline 43403 & i.MP9702 & 8 & 4481 & 24.70 & 0.54 \\
\hline 43403 & z.MP9801 & 2 & 1080 & 22.47 & 0.41 \\
\hline 46649 & u.MP9301 & 5 & 2801 & 24.96 & 0.79 \\
\hline 46649 & g.MP9401 & 3 & 1020 & 24.76 & 0.62 \\
\hline 46649 & r.MP9601 & 4 & 1992 & 24.68 & 0.82 \\
\hline 46649 & i.MP9702 & 9 & 5041 & 24.67 & 0.51 \\
\hline 46649 & z.MP9801 & 4 & 2241 & 23.44 & 0.77 \\
\hline 47981 & u.MP9301 & 5 & 2800 & 25.20 & 1.21 \\
\hline 47981 & g.MP9401 & 3 & 1020 & 25.08 & 1.17 \\
\hline 47981 & r.MP9601 & 4 & 1992 & 24.86 & 0.65 \\
\hline 47981 & i.MP9702 & 9 & 5041 & 24.81 & 0.73 \\
\hline 47981 & z.MP9801 & 4 & 2240 & 23.27 & 0.53 \\
\hline 50492 & u.MP9301 & 5 & 2801 & 25.16 & 0.56 \\
\hline 50492 & g.MP9401 & 3 & 1080 & 24.91 & 0.56 \\
\hline 50492 & r.MP9601 & 6 & 3001 & 24.87 & 0.42 \\
\hline 50492 & i.MP9702 & 8 & 4481 & 24.58 & 0.70 \\
\hline 50492 & z.MP9801 & 4 & 2241 & 23.11 & 0.58 \\
\hline 50514 & u.MP9301 & 5 & 2801 & 25.24 & 0.95 \\
\hline 50514 & g.MP9401 & 3 & 1020 & 25.04 & 0.78 \\
\hline 50514 & r.MP9601 & 5 & 2491 & 24.67 & 0.71 \\
\hline 50514 & i.MP9702 & 8 & 4481 & 24.80 & 0.72 \\
\hline 50514 & z.MP9801 & 4 & 2241 & 23.20 & 0.59 \\
\hline 52480 & u.MP9301 & 5 & 2600 & 25.18 & 0.71 \\
\hline 52480 & g.MP9401 & 3 & 900 & 25.00 & 0.79 \\
\hline 52480 & r.MP9601 & 3 & 1380 & 24.56 & 0.94 \\
\hline 52480 & i.MP9702 & 10 & 5601 & 24.71 & 0.76 \\
\hline 52480 & z.MP9901 & 4 & 2241 & 22.91 & 0.81 \\
\hline 53436 & u.MP9301 & 7 & 3641 & 25.30 & 0.89 \\
\hline 53436 & g.MP9401 & 3 & 900 & 24.88 & 0.83 \\
\hline 53436 & r.MP9601 & 7 & 3221 & 25.04 & 1.04 \\
\hline 53436 & i.MP9702 & 8 & 4481 & 24.54 & 0.74 \\
\hline 53495 & u.MP9301 & 7 & 3641 & 25.30 & 0.89 \\
\hline 53495 & g.MP9401 & 3 & 900 & 24.88 & 0.83 \\
\hline 53495 & r.MP9601 & 7 & 3221 & 25.04 & 1.04 \\
\hline 53495 & i.MP9702 & 8 & 4481 & 24.54 & 0.74 \\
\hline 54795 & u.MP9301 & 5 & 2801 & 25.13 & 0.94 \\
\hline 54795 & g.MP9401 & 3 & 1020 & 24.97 & 1.13 \\
\hline 54795 & r.MP9601 & 3 & 1494 & 24.16 & 0.74 \\
\hline 54795 & i.MP9702 & 8 & 4481 & 24.55 & 0.64 \\
\hline 54795 & z.MP9801 & 4 & 2240 & 23.18 & 0.70 \\
\hline 55181 & u.MP9301 & 5 & 2801 & 25.02 & 1.18 \\
\hline 55181 & g.MP9401 & 3 & 1080 & 24.94 & 1.19 \\
\hline 55181 & r.MP9601 & 6 & 3001 & 24.75 & 0.74 \\
\hline 55181 & i.MP9702 & 8 & 4481 & 24.56 & 0.62 \\
\hline 55181 & z.MP9801 & 4 & 2240 & 22.99 & 0.67 \\
\hline
\end{tabular}




\begin{tabular}{cccccc}
\hline CODEX ID & Filter & N. of exp. & $\begin{array}{c}\text { expos. time } \\
{[\mathrm{s}]}\end{array}$ & $\begin{array}{c}m_{\lim } \\
{[\mathrm{AB} \text { mag }]}\end{array}$ & $\begin{array}{c}\text { Seeing } \\
{\left[{ }^{\prime \prime}\right]}\end{array}$ \\
\hline \hline 59915 & u.MP9301 & 5 & 2800 & 25.08 & 0.96 \\
59915 & g.MP9401 & 3 & 1020 & 24.90 & 0.89 \\
59915 & r.MP9601 & 3 & 1494 & 24.50 & 0.91 \\
59915 & i.MP9702 & 8 & 4481 & 24.80 & 0.62 \\
59915 & z.MP9801 & 4 & 2240 & 23.33 & 0.76 \\
\hline 64232 & u.MP9301 & 5 & 2800 & 25.20 & 0.90 \\
64232 & g.MP9401 & 3 & 1020 & 25.05 & 0.65 \\
64232 & r.MP9601 & 3 & 1494 & 24.59 & 0.84 \\
64232 & i.MP9702 & 6 & 3481 & 24.60 & 0.79 \\
64232 & z.MP9801 & 4 & 2240 & 23.17 & 0.74 \\
\hline \hline
\end{tabular}

Table D2: CODEX sample S-II covered by CFHTLS Wide (see Erben et al. 2013)

\begin{tabular}{|c|c|c|c|c|c|c|}
\hline$\overline{\text { CODEX ID }}$ & $\begin{array}{l}\text { CFHTLS Wide } \\
\text { field }\end{array}$ & Filter & $\begin{array}{c}\text { N. of exp. } \\
{[\mathrm{s}]}\end{array}$ & $\begin{array}{c}\text { expos. time } \\
{[\mathrm{AB} \text { mag] }}\end{array}$ & $\begin{array}{c}m_{\lim } \\
{\left[{ }^{\prime \prime}\right]}\end{array}$ & Seeing \\
\hline 13311 & "W3m3m1 & u.MP9301 & $\overline{5}$ & 3001 & 25.05 & 0.93 \\
\hline 13311 & $\mathrm{~W} 3 \mathrm{~m} 3 \mathrm{~m} 1$ & g.MP9401 & 5 & 2501 & 25.26 & 0.84 \\
\hline 13311 & $\mathrm{~W} 3 \mathrm{~m} 3 \mathrm{~m} 1$ & r.MP9601 & 4 & 2001 & 24.80 & 0.72 \\
\hline 13311 & $\mathrm{~W} 3 \mathrm{~m} 3 \mathrm{~m} 1$ & i.MP9701 & 7 & 4341 & 24.56 & 0.94 \\
\hline 13311 & $\mathrm{~W} 3 \mathrm{~m} 3 \mathrm{~m} 1$ & z.MP9801 & 6 & 3601 & 23.39 & 0.56 \\
\hline 13315 & $\mathrm{~W} 3 \mathrm{~m} 0 \mathrm{~m} 1$ & u.MP9301 & 5 & 3001 & 24.99 & 0.78 \\
\hline 13315 & $\mathrm{~W} 3 \mathrm{~m} 0 \mathrm{~m} 1$ & g.MP9401 & 5 & 2501 & 25.59 & 0.76 \\
\hline 13315 & $\mathrm{~W} 3 \mathrm{~m} 0 \mathrm{~m} 1$ & r.MP9601 & 4 & 2001 & 25.00 & 0.61 \\
\hline 13315 & $\mathrm{~W} 3 \mathrm{~m} 0 \mathrm{~m} 1$ & i.MP9701 & 7 & 4306 & 24.63 & 0.54 \\
\hline 13315 & $\mathrm{~W} 3 \mathrm{~m} 0 \mathrm{~m} 1$ & z.MP9801 & 6 & 3601 & 23.39 & 0.62 \\
\hline 13380 & W3p3p1 & u.MP9301 & 5 & 3001 & 25.23 & 0.88 \\
\hline 13380 & W3p3p1 & g.MP9401 & 5 & 2501 & 25.70 & 0.97 \\
\hline 13380 & W3p3p1 & r.MP9601 & 4 & 2001 & 25.06 & 0.76 \\
\hline 13380 & W3p3p1 & i.MP9701 & 7 & 4306 & 24.73 & 0.83 \\
\hline 13380 & W3p3p1 & z.MP9801 & 6 & 3601 & 23.07 & 0.68 \\
\hline 13390 & $\mathrm{~W} 3 \mathrm{~m} 0 \mathrm{~m} 0$ & u.MP9301 & 5 & 3001 & 25.02 & 0.97 \\
\hline 13390 & $\mathrm{~W} 3 \mathrm{~m} 0 \mathrm{~m} 0$ & g.MP9401 & 5 & 2501 & 25.53 & 0.94 \\
\hline 13390 & $\mathrm{~W} 3 \mathrm{~m} 0 \mathrm{~m} 0$ & r.MP9601 & 4 & 2001 & 24.77 & 0.87 \\
\hline 13390 & $\mathrm{~W} 3 \mathrm{~m} 0 \mathrm{~m} 0$ & i.MP9701 & 7 & 4341 & 24.41 & 0.94 \\
\hline 13390 & $\mathrm{~W} 3 \mathrm{~m} 0 \mathrm{~m} 0$ & z.MP9801 & 5 & 3001 & 23.12 & 0.76 \\
\hline 13390 & $\mathrm{~W} 3 \mathrm{~m} 1 \mathrm{~m} 0$ & u.MP9301 & 5 & 3001 & 24.91 & 0.69 \\
\hline 13390 & $\mathrm{~W} 3 \mathrm{~m} 1 \mathrm{~m} 0$ & g.MP9401 & 6 & 3001 & 25.66 & 0.99 \\
\hline 13390 & $\mathrm{~W} 3 \mathrm{~m} 1 \mathrm{~m} 0$ & r.MP9601 & 4 & 2001 & 25.09 & 0.66 \\
\hline 13390 & $\mathrm{~W} 3 \mathrm{~m} 1 \mathrm{~m} 0$ & i.MP9701 & 7 & 4306 & 24.24 & 0.53 \\
\hline 13390 & $\mathrm{~W} 3 \mathrm{~m} 1 \mathrm{~m} 0$ & z.MP9801 & 4 & 2401 & 23.01 & 0.71 \\
\hline 13391 & $\mathrm{~W} 3 \mathrm{p} 3 \mathrm{~m} 0$ & u.MP9301 & 5 & 3001 & 25.26 & 0.99 \\
\hline 13391 & $\mathrm{~W} 3 \mathrm{p} 3 \mathrm{~m} 0$ & g.MP9401 & 5 & 2501 & 25.67 & 0.97 \\
\hline 13391 & $\mathrm{~W} 3 \mathrm{p} 3 \mathrm{~m} 0$ & r.MP9601 & 4 & 2001 & 25.06 & 0.76 \\
\hline 13391 & $\mathrm{~W} 3 \mathrm{p} 3 \mathrm{~m} 0$ & i.MP9701 & 7 & 4306 & 24.74 & 0.71 \\
\hline 13391 & $\mathrm{~W} 3 \mathrm{p} 3 \mathrm{~m} 0$ & z.MP9801 & 6 & 3601 & 23.61 & 0.73 \\
\hline 13400 & $\mathrm{~W} 3 \mathrm{p} 3 \mathrm{~m} 0$ & u.MP9301 & 5 & 3001 & 25.26 & 0.99 \\
\hline 13400 & $\mathrm{~W} 3 \mathrm{p} 3 \mathrm{~m} 0$ & g.MP9401 & 5 & 2501 & 25.67 & 0.97 \\
\hline 13400 & $\mathrm{~W} 3 \mathrm{p} 3 \mathrm{~m} 0$ & r.MP9601 & 4 & 2001 & 25.06 & 0.76 \\
\hline 13400 & $\mathrm{~W} 3 \mathrm{p} 3 \mathrm{~m} 0$ & i.MP9701 & 7 & 4306 & 24.74 & 0.71 \\
\hline 13400 & $\mathrm{~W} 3 \mathrm{p} 3 \mathrm{~m} 0$ & z.MP9801 & 6 & 3601 & 23.61 & 0.73 \\
\hline 13400 & W3p3m1 & u.MP9301 & 5 & 3001 & 25.24 & 0.89 \\
\hline 13400 & $\mathrm{~W} 3 \mathrm{p} 3 \mathrm{~m} 1$ & g.MP9401 & 6 & 3001 & 25.71 & 0.89 \\
\hline 13400 & W3p3m1 & r.MP9601 & 4 & 2001 & 25.05 & 0.79 \\
\hline 13400 & $\mathrm{~W} 3 \mathrm{p} 3 \mathrm{~m} 1$ & i.MP9701 & 6 & 3691 & 24.87 & 0.85 \\
\hline 13400 & W3p3m1 & i.MP9702 & 7 & 4306 & 24.71 & 0.68 \\
\hline 13400 & W3p3m1 & z.MP9801 & 6 & 3601 & 23.64 & 0.64 \\
\hline
\end{tabular}




\begin{tabular}{|c|c|c|c|c|c|c|}
\hline CODEX ID & $\begin{array}{l}\text { CFHTLS Wide } \\
\text { field } \\
\end{array}$ & Filter & $\begin{array}{c}\text { N. of exp. } \\
\text { [s] }\end{array}$ & $\begin{array}{c}\text { expos. time } \\
\text { [AB mag] }\end{array}$ & $\begin{array}{c}m_{\lim } \\
{\left[{ }^{\prime \prime}\right]}\end{array}$ & Seeing \\
\hline 17449 & $\mathrm{~W} 3 \mathrm{~m} 1 \mathrm{~m} 2$ & u.MP9301 & 5 & 3001 & 24.48 & 0.86 \\
\hline 17449 & $\mathrm{~W} 3 \mathrm{~m} 1 \mathrm{~m} 2$ & g.MP9401 & 4 & 2001 & 25.40 & 0.88 \\
\hline 17449 & $\mathrm{~W} 3 \mathrm{~m} 1 \mathrm{~m} 2$ & r.MP9601 & 5 & 2501 & 24.93 & 0.65 \\
\hline 17449 & $\mathrm{~W} 3 \mathrm{~m} 1 \mathrm{~m} 2$ & i.MP9701 & 7 & 4306 & 24.34 & 0.65 \\
\hline 17449 & $\mathrm{~W} 3 \mathrm{~m} 1 \mathrm{~m} 2$ & z.MP9801 & 6 & 3601 & 23.45 & 0.67 \\
\hline 17449 & $\mathrm{~W} 3 \mathrm{~m} 1 \mathrm{~m} 3$ & u.MP9301 & 5 & 3001 & 24.61 & 0.75 \\
\hline 17449 & $\mathrm{~W} 3 \mathrm{~m} 1 \mathrm{~m} 3$ & g.MP9401 & 5 & 2501 & 25.64 & 0.86 \\
\hline 17449 & $\mathrm{~W} 3 \mathrm{~m} 1 \mathrm{~m} 3$ & r.MP9601 & 4 & 2001 & 24.84 & 0.70 \\
\hline 17449 & $\mathrm{~W} 3 \mathrm{~m} 1 \mathrm{~m} 3$ & i.MP9701 & 7 & 4307 & 24.41 & 0.66 \\
\hline 17449 & $\mathrm{~W} 3 \mathrm{~m} 1 \mathrm{~m} 3$ & z.MP9801 & 6 & 3601 & 23.41 & 0.59 \\
\hline 17453 & $\mathrm{~W} 3 \mathrm{~m} 2 \mathrm{~m} 2$ & u.MP9301 & 5 & 3001 & 25.11 & 0.77 \\
\hline 17453 & $\mathrm{~W} 3 \mathrm{~m} 2 \mathrm{~m} 2$ & g.MP9401 & 5 & 2501 & 25.55 & 0.89 \\
\hline 17453 & $\mathrm{~W} 3 \mathrm{~m} 2 \mathrm{~m} 2$ & r.MP9601 & 4 & 2001 & 24.97 & 0.62 \\
\hline 17453 & $\mathrm{~W} 3 \mathrm{~m} 2 \mathrm{~m} 2$ & i.MP9701 & 7 & 4306 & 24.46 & 0.65 \\
\hline 17453 & $\mathrm{~W} 3 \mathrm{~m} 2 \mathrm{~m} 2$ & z.MP9801 & 6 & 3601 & 23.43 & 0.64 \\
\hline 17453 & $\mathrm{~W} 3 \mathrm{~m} 2 \mathrm{~m} 3$ & u.MP9301 & 9 & 5402 & 25.57 & 0.86 \\
\hline 17453 & $\mathrm{~W} 3 \mathrm{~m} 2 \mathrm{~m} 3$ & g.MP9401 & 6 & 2501 & 25.62 & 0.89 \\
\hline 17453 & $\mathrm{~W} 3 \mathrm{~m} 2 \mathrm{~m} 3$ & r.MP9601 & 5 & 2001 & 24.97 & 0.80 \\
\hline 17453 & $\mathrm{~W} 3 \mathrm{~m} 2 \mathrm{~m} 3$ & i.MP9701 & 7 & 4306 & 24.40 & 0.73 \\
\hline 17453 & $\mathrm{~W} 3 \mathrm{~m} 2 \mathrm{~m} 3$ & z.MP9801 & 6 & 3601 & 23.22 & 0.82 \\
\hline 54652 & $\mathrm{~W} 4 \mathrm{~m} 3 \mathrm{p} 3$ & u.MP9301 & 5 & 3000 & 25.34 & 0.90 \\
\hline 54652 & $\mathrm{~W} 4 \mathrm{~m} 3 \mathrm{p} 3$ & g.MP9401 & 6 & 3000 & 25.70 & 0.71 \\
\hline 54652 & $\mathrm{~W} 4 \mathrm{~m} 3 \mathrm{p} 3$ & r.MP9601 & 4 & 2000 & 24.72 & 0.76 \\
\hline 54652 & $\mathrm{~W} 4 \mathrm{~m} 3 \mathrm{p} 3$ & i.MP9702 & 7 & 4306 & 24.70 & 0.57 \\
\hline 54652 & $\mathrm{~W} 4 \mathrm{~m} 3 \mathrm{p} 3$ & z.MP9801 & 6 & 3600 & 23.32 & 0.62 \\
\hline 56934 & W2m0p3 & u.MP9301 & 5 & 3001 & 25.25 & 0.82 \\
\hline 56934 & $\mathrm{~W} 2 \mathrm{~m} 0 \mathrm{p} 3$ & g.MP9401 & 5 & 2500 & 25.26 & 0.71 \\
\hline 56934 & $\mathrm{~W} 2 \mathrm{~m} 0 \mathrm{p} 3$ & r.MP9601 & 6 & 3000 & 25.04 & 0.70 \\
\hline 56934 & $\mathrm{~W} 2 \mathrm{~m} 0 \mathrm{p} 3$ & i.MP9701 & 7 & 4305 & 24.36 & 0.51 \\
\hline 56934 & $\mathrm{~W} 2 \mathrm{~m} 0 \mathrm{p} 3$ & z.MP9801 & 6 & 3600 & 23.27 & 0.78 \\
\hline 56934 & $\mathrm{~W} 2 \mathrm{~m} 1 \mathrm{p} 3$ & u.MP9301 & 5 & 3000 & 25.38 & 1.07 \\
\hline 56934 & $\mathrm{~W} 2 \mathrm{~m} 1 \mathrm{p} 3$ & g.MP9401 & 5 & 2500 & 25.67 & 0.75 \\
\hline 56934 & $\mathrm{~W} 2 \mathrm{~m} 1 \mathrm{p} 3$ & r.MP9601 & 6 & 3001 & 25.16 & 0.66 \\
\hline 56934 & $\mathrm{~W} 2 \mathrm{~m} 1 \mathrm{p} 3$ & i.MP9701 & 5 & 3075 & 24.48 & 0.63 \\
\hline 56934 & $\mathrm{~W} 2 \mathrm{~m} 1 \mathrm{p} 3$ & z.MP9801 & 6 & 3600 & 23.46 & 0.69 \\
\hline 57017 & $\mathrm{~W} 2 \mathrm{p} 2 \mathrm{p} 3$ & u.MP9301 & 5 & 3001 & 25.19 & 0.81 \\
\hline 57017 & $\mathrm{~W} 2 \mathrm{p} 2 \mathrm{p} 3$ & g.MP9401 & 5 & 2500 & 25.72 & 0.93 \\
\hline 57017 & $\mathrm{~W} 2 \mathrm{p} 2 \mathrm{p} 3$ & r.MP9601 & 5 & 2500 & 25.04 & 0.82 \\
\hline 57017 & $\mathrm{~W} 2 \mathrm{p} 2 \mathrm{p} 3$ & i.MP9701 & 7 & 4305 & 24.62 & 0.78 \\
\hline 57017 & $\mathrm{~W} 2 \mathrm{p} 2 \mathrm{p} 3$ & z.MP9801 & 7 & 4200 & 23.58 & 0.83 \\
\hline 60076 & W1m1p3 & u.MP9301 & 5 & 3001 & 25.06 & 0.85 \\
\hline 60076 & $\mathrm{~W} 1 \mathrm{~m} 1 \mathrm{p} 3$ & g.MP9401 & 5 & 2501 & 25.42 & 0.94 \\
\hline 60076 & $\mathrm{~W} 1 \mathrm{~m} 1 \mathrm{p} 3$ & r.MP9601 & 4 & 2000 & 24.89 & 0.83 \\
\hline 60076 & $\mathrm{~W} 1 \mathrm{~m} 1 \mathrm{p} 3$ & i.MP9701 & 7 & 4306 & 24.64 & 0.76 \\
\hline 60076 & W1m1p3 & z.MP9801 & 10 & 6001 & 23.56 & 0.72 \\
\hline 60131 & W1p1m2 & u.MP9301 & 5 & 3000 & 25.38 & 1.03 \\
\hline 60131 & $\mathrm{~W} 1 \mathrm{p} 1 \mathrm{~m} 2$ & g.MP9401 & 5 & 2500 & 25.60 & 0.76 \\
\hline 60131 & $\mathrm{~W} 1 \mathrm{p} 1 \mathrm{~m} 2$ & r.MP9601 & 4 & 2000 & 24.86 & 0.69 \\
\hline 60131 & $\mathrm{~W} 1 \mathrm{p} 1 \mathrm{~m} 2$ & i.MP9701 & 8 & 4921 & 24.86 & 0.70 \\
\hline 60131 & $\mathrm{~W} 1 \mathrm{p} 1 \mathrm{~m} 2$ & z.MP9801 & 6 & 3600 & 23.50 & 0.72 \\
\hline 60131 & $\mathrm{~W} 1 \mathrm{p} 2 \mathrm{~m} 2$ & u.MP9301 & 6 & 3601 & 25.44 & 1.04 \\
\hline 60131 & $\mathrm{~W} 1 \mathrm{p} 2 \mathrm{~m} 2$ & g.MP9401 & 5 & 2500 & 25.61 & 0.73 \\
\hline 60131 & $\mathrm{~W} 1 \mathrm{p} 2 \mathrm{~m} 2$ & r.MP9601 & 4 & 2000 & 24.79 & 0.78 \\
\hline 60131 & $\mathrm{~W} 1 \mathrm{p} 2 \mathrm{~m} 2$ & i.MP9701 & 7 & 4306 & 24.76 & 0.64 \\
\hline 60131 & $\mathrm{~W} 1 \mathrm{p} 2 \mathrm{~m} 2$ & z.MP9801 & 7 & 4200 & 23.36 & 0.89 \\
\hline
\end{tabular}




\begin{tabular}{|c|c|c|c|c|c|c|}
\hline CODEX ID & $\begin{array}{l}\text { CFHTLS Wide } \\
\text { field }\end{array}$ & Filter & $\begin{array}{c}\text { N. of exp. } \\
{[\mathrm{s}]}\end{array}$ & $\begin{array}{c}\text { expos. time } \\
\text { [AB mag] }\end{array}$ & $\begin{array}{c}m_{\lim } \\
{\left[{ }^{\prime \prime}\right]}\end{array}$ & Seeing \\
\hline 60155 & W1p4p2 & u.MP9301 & 5 & 3001 & 25.23 & 0.76 \\
\hline 60155 & W1p4p2 & g.MP9401 & 5 & 2500 & 25.52 & 0.86 \\
\hline 60155 & $\mathrm{~W} 1 \mathrm{p} 4 \mathrm{p} 2$ & r.MP9601 & 4 & 2000 & 24.76 & 0.60 \\
\hline 60155 & W1p4p2 & i.MP9701 & 7 & 4341 & 24.41 & 0.87 \\
\hline 60155 & $\mathrm{~W} 1 \mathrm{p} 4 \mathrm{p} 2$ & i.MP9702 & 10 & 6150 & 24.81 & 0.63 \\
\hline 60155 & $\mathrm{~W} 1 \mathrm{p} 4 \mathrm{p} 2$ & z.MP9801 & 6 & 3601 & 23.43 & 0.55 \\
\hline 64565 & $\mathrm{~W} 1 \mathrm{~m} 4 \mathrm{~m} 3$ & u.MP9301 & 5 & 3000 & 25.22 & 0.84 \\
\hline 64565 & $\mathrm{~W} 1 \mathrm{~m} 4 \mathrm{~m} 3$ & g.MP9401 & 7 & 3500 & 25.68 & 0.84 \\
\hline 64565 & $\mathrm{~W} 1 \mathrm{~m} 4 \mathrm{~m} 3$ & r.MP9601 & 4 & 2000 & 24.89 & 0.94 \\
\hline 64565 & $\mathrm{~W} 1 \mathrm{~m} 4 \mathrm{~m} 3$ & i.MP9701 & 7 & 4306 & 24.46 & 0.59 \\
\hline 64565 & $\mathrm{~W} 1 \mathrm{~m} 4 \mathrm{~m} 3$ & z.MP9801 & 6 & 3600 & 23.55 & 0.82 \\
\hline 64636 & $\mathrm{~W} 1 \mathrm{p} 1 \mathrm{~m} 2$ & u.MP9301 & 5 & 3000 & 25.38 & 1.03 \\
\hline 64636 & $\mathrm{~W} 1 \mathrm{p} 1 \mathrm{~m} 2$ & g.MP9401 & 5 & 2500 & 25.60 & 0.76 \\
\hline 64636 & $\mathrm{~W} 1 \mathrm{p} 1 \mathrm{~m} 2$ & r.MP9601 & 4 & 2000 & 24.86 & 0.69 \\
\hline 64636 & $\mathrm{~W} 1 \mathrm{p} 1 \mathrm{~m} 2$ & i.MP9701 & 8 & 4921 & 24.86 & 0.70 \\
\hline 64636 & $\mathrm{~W} 1 \mathrm{p} 1 \mathrm{~m} 2$ & z.MP9801 & 6 & 3600 & 23.50 & 0.72 \\
\hline 64636 & $\mathrm{~W} 1 \mathrm{p} 2 \mathrm{~m} 2$ & u.MP9301 & 6 & 3601 & 25.44 & 1.04 \\
\hline 64636 & $\mathrm{~W} 1 \mathrm{p} 2 \mathrm{~m} 2$ & g.MP9401 & 5 & 2500 & 25.61 & 0.73 \\
\hline 64636 & $\mathrm{~W} 1 \mathrm{p} 2 \mathrm{~m} 2$ & r.MP9601 & 4 & 2000 & 24.79 & 0.78 \\
\hline 64636 & $\mathrm{~W} 1 \mathrm{p} 2 \mathrm{~m} 2$ & i.MP9701 & 7 & 4306 & 24.76 & 0.64 \\
\hline 64636 & $\mathrm{~W} 1 \mathrm{p} 2 \mathrm{~m} 2$ & z.MP9801 & 7 & 4200 & 23.36 & 0.89 \\
\hline 210288 & $\mathrm{~W} 3 \mathrm{p} 2 \mathrm{p} 2$ & u.MP9301 & 5 & 3001 & 25.20 & 0.68 \\
\hline 210288 & $\mathrm{~W} 3 \mathrm{p} 2 \mathrm{p} 2$ & g.MP9401 & 5 & 2501 & 25.53 & 0.81 \\
\hline 210288 & W3p2p2 & r.MP9601 & 4 & 2001 & 24.88 & 0.79 \\
\hline 210288 & W3p2p2 & i.MP9701 & 7 & 4307 & 24.51 & 0.54 \\
\hline 210288 & $\mathrm{~W} 3 \mathrm{p} 2 \mathrm{p} 2$ & z.MP9801 & 6 & 3601 & 22.95 & 0.53 \\
\hline 210288 & W3p2p3 & u.MP9301 & 5 & 3001 & 25.25 & 0.99 \\
\hline 210288 & W3p2p3 & g.MP9401 & 5 & 2501 & 25.49 & 0.74 \\
\hline 210288 & W3p2p3 & r.MP9601 & 4 & 2001 & 24.93 & 0.67 \\
\hline 210288 & $\mathrm{~W} 3 \mathrm{p} 2 \mathrm{p} 3$ & i.MP9701 & 7 & 4306 & 24.56 & 0.69 \\
\hline 210288 & $\mathrm{~W} 3 \mathrm{p} 2 \mathrm{p} 3$ & z.MP9801 & 6 & 3601 & 23.82 & 0.63 \\
\hline 210306 & W3p1p1 & u.MP9301 & 5 & 3001 & 25.28 & 0.93 \\
\hline 210306 & W3p1p1 & g.MP9401 & 5 & 2501 & 25.66 & 0.79 \\
\hline 210306 & W3p1p1 & r.MP9601 & 4 & 2001 & 24.94 & 0.84 \\
\hline 210306 & W3p1p1 & i.MP9701 & 7 & 4306 & 24.64 & 0.71 \\
\hline 210306 & W3p1p1 & z.MP9801 & 6 & 3601 & 23.63 & 0.72 \\
\hline 210306 & W3p2p1 & u.MP9301 & 5 & 3001 & 25.19 & 0.78 \\
\hline 210306 & W3p2p1 & g.MP9401 & 6 & 3001 & 25.69 & 0.84 \\
\hline 210306 & W3p2p1 & r.MP9601 & 4 & 2001 & 24.94 & 0.64 \\
\hline 210306 & W3p2p1 & i.MP9701 & 9 & 5537 & 24.56 & 0.70 \\
\hline 210306 & W3p2p1 & z.MP9801 & 7 & 4201 & 23.13 & 0.57 \\
\hline
\end{tabular}


Table D3: CODEX sample S-III $(z \lesssim 0.4)$

\begin{tabular}{lccccc}
\hline \hline CODEX ID & Filter & N. of exp. & $\begin{array}{c}\text { expos. time } \\
{[\mathrm{s}]}\end{array}$ & $\begin{array}{c}m_{\lim } \\
{[\mathrm{AB} \text { mag }]}\end{array}$ & $\begin{array}{c}\text { Seeing } \\
{\left[{ }^{\prime \prime}\right]}\end{array}$ \\
\hline \hline 24925 & u.MP9301 & 5 & 2801 & 25.02 & 1.18 \\
24925 & g.MP9401 & 2 & 680 & 24.72 & 0.93 \\
24925 & r.MP9601 & 3 & 1500 & 24.39 & 0.87 \\
24925 & i.MP9702 & 9 & 5042 & 24.89 & 0.73 \\
24925 & z.MP9801 & 4 & 2241 & 22.96 & 0.76 \\
\hline 27955 & u.MP9301 & 7 & 3921 & 25.42 & 0.94 \\
27955 & r.MP9601 & 4 & 1993 & 24.51 & 0.61 \\
27955 & i.MP9702 & 8 & 4481 & 24.62 & 0.50 \\
27955 & z.MP9801 & 4 & 2241 & 23.13 & 0.49 \\
\hline 46647 & u.MP9301 & 5 & 2801 & 24.96 & 0.79 \\
46647 & g.MP9401 & 3 & 1020 & 24.76 & 0.62 \\
46647 & r.MP9601 & 4 & 1992 & 24.68 & 0.82 \\
46647 & i.MP9702 & 9 & 5041 & 24.67 & 0.51 \\
46647 & z.MP9801 & 4 & 2241 & 23.44 & 0.77 \\
\hline 54796 & u.MP9301 & 5 & 2801 & 25.13 & 0.94 \\
54796 & g.MP9401 & 3 & 1020 & 24.97 & 1.13 \\
54796 & r.MP9601 & 3 & 1494 & 24.16 & 0.74 \\
54796 & i.MP9702 & 8 & 4481 & 24.55 & 0.64 \\
54796 & z.MP9801 & 4 & 2240 & 23.18 & 0.70 \\
\hline \hline
\end{tabular}


Table D4: Additional CODEX galaxy clusters observed in three bands

\begin{tabular}{|c|c|c|c|c|c|}
\hline CODEX ID & Filter & N. of exp. & $\begin{array}{c}\text { expos. time } \\
{[\mathrm{s}]}\end{array}$ & $\begin{array}{c}m_{\lim } \\
{[\mathrm{AB} \mathrm{mag}]}\end{array}$ & $\begin{array}{c}\text { Seeing } \\
{\left[{ }^{\prime \prime}\right]}\end{array}$ \\
\hline 16463 & u.MP9301 & 7 & 3641 & 25.40 & 1.09 \\
\hline 16463 & i.MP9702 & 8 & 4482 & 24.74 & 0.57 \\
\hline 16463 & z.MP9801 & 6 & 3241 & 23.47 & 0.77 \\
\hline 16470 & u.MP9301 & 7 & 3641 & 25.40 & 1.09 \\
\hline 16470 & i.MP9702 & 8 & 4482 & 24.74 & 0.57 \\
\hline 16470 & z.MP9801 & 6 & 3241 & 23.47 & 0.77 \\
\hline 20621 & g.MP9401 & 4 & 841 & 24.82 & 1.14 \\
\hline 20621 & r.MP9601 & 3 & 631 & 24.16 & 1.06 \\
\hline 20621 & i.MP9702 & 11 & 6162 & 24.84 & 0.61 \\
\hline 20622 & g.MP9401 & 4 & 841 & 24.82 & 1.14 \\
\hline 20622 & r.MP9601 & 3 & 631 & 24.16 & 1.06 \\
\hline 20622 & i.MP9702 & 11 & 6162 & 24.84 & 0.61 \\
\hline 24747 & u.MP9301 & 5 & 2601 & 25.20 & 0.73 \\
\hline 24747 & i.MP9702 & 7 & 3921 & 24.64 & 0.61 \\
\hline 24747 & z.MP9801 & 4 & 2241 & 22.81 & 0.90 \\
\hline 25094 & g.MP9401 & 3 & 630 & 24.81 & 0.74 \\
\hline 25094 & r.MP9601 & 3 & 630 & 24.29 & 0.75 \\
\hline 25094 & i.MP9702 & 1 & 560 & 23.41 & 0.51 \\
\hline 25252 & g.MP9401 & 3 & 630 & 24.93 & 1.23 \\
\hline 25252 & r.MP9601 & 3 & 630 & 24.34 & 1.07 \\
\hline 25252 & i.MP9702 & 8 & 4482 & 24.74 & 0.60 \\
\hline 29249 & u.MP9301 & 5 & 2601 & 25.20 & 0.73 \\
\hline 29249 & i.MP9702 & 7 & 3921 & 24.64 & 0.61 \\
\hline 29249 & z.MP9801 & 4 & 2241 & 22.81 & 0.90 \\
\hline 37287 & u.MP9301 & 3 & 1681 & 24.81 & 1.13 \\
\hline 37287 & i.MP9702 & 8 & 4481 & 24.55 & 0.64 \\
\hline 37287 & z.MP9801 & 4 & 2241 & 22.89 & 0.50 \\
\hline 39323 & g.MP9401 & 3 & 630 & 24.79 & 0.67 \\
\hline 39323 & r.MP9601 & 3 & 630 & 24.12 & 0.56 \\
\hline 39323 & i.MP9702 & 8 & 4481 & 24.51 & 0.56 \\
\hline 39326 & g.MP9401 & 3 & 630 & 24.79 & 0.67 \\
\hline 39326 & r.MP9601 & 3 & 630 & 24.12 & 0.56 \\
\hline 39326 & i.MP9702 & 8 & 4481 & 24.51 & 0.56 \\
\hline 39879 & g.MP9401 & 3 & 630 & 24.86 & 0.73 \\
\hline 39879 & r.MP9601 & 3 & 630 & 24.33 & 0.61 \\
\hline 39879 & i.MP9702 & 8 & 4481 & 24.55 & 0.67 \\
\hline 43881 & g.MP9401 & 5 & 736 & 24.79 & 0.66 \\
\hline 43881 & r.MP9601 & 4 & 921 & 24.35 & 1.21 \\
\hline 43881 & i.MP9702 & 6 & 715 & 23.90 & 0.84 \\
\hline 46328 & g.MP9401 & 3 & 1020 & 25.05 & 0.96 \\
\hline 46328 & r.MP9601 & 3 & 1500 & 24.66 & 0.62 \\
\hline 46328 & i.MP9702 & 7 & 3921 & 24.82 & 0.66 \\
\hline 50502 & g.MP9401 & 3 & 1020 & 25.10 & 0.91 \\
\hline 50502 & r.MP9601 & 3 & 1500 & 24.80 & 0.56 \\
\hline 50502 & i.MP9702 & 8 & 4481 & 24.77 & 0.77 \\
\hline 52484 & g.MP9401 & 3 & 630 & 24.83 & 0.99 \\
\hline 52484 & r.MP9601 & 5 & 1050 & 24.53 & 0.93 \\
\hline 52484 & i.MP9702 & 8 & 4481 & 24.69 & 0.47 \\
\hline 57892 & g.MP9401 & 1 & 260 & 23.59 & 1.02 \\
\hline 57892 & r.MP9601 & 5 & 1500 & 24.49 & 0.99 \\
\hline 57892 & i.MP9702 & 1 & 560 & 23.75 & 0.90 \\
\hline 58014 & g.MP9401 & 5 & 1050 & 25.05 & 0.81 \\
\hline 58014 & r.MP9601 & 3 & 630 & 24.28 & 0.73 \\
\hline 58014 & i.MP9702 & 10 & 5601 & 24.83 & 0.85 \\
\hline
\end{tabular}




\begin{tabular}{cccccc}
\hline CODEX ID & Filter & N. of exp. & $\begin{array}{c}\text { expos. time } \\
{[\mathrm{s}]}\end{array}$ & $\begin{array}{c}m_{\lim } \\
{[\mathrm{AB} \text { mag }]}\end{array}$ & $\begin{array}{c}\text { Seeing } \\
{\left[{ }^{\prime \prime}\right]}\end{array}$ \\
\hline \hline 58114 & g.MP9401 & 3 & 630 & 24.82 & 0.92 \\
58114 & r.MP9601 & 3 & 630 & 24.28 & 0.81 \\
58114 & i.MP9702 & 10 & 5601 & 24.71 & 0.70 \\
\hline 64360 & g.MP9401 & 3 & 1020 & 25.06 & 0.88 \\
64360 & r.MP9601 & 3 & 540 & 23.79 & 0.89 \\
64360 & i.MP9702 & 9 & 5041 & 24.80 & 0.69 \\
\hline 219599 & g.MP9401 & 3 & 630 & 24.81 & 0.74 \\
219599 & r.MP9601 & 3 & 630 & 24.29 & 0.75 \\
219599 & i.MP9702 & 1 & 560 & 23.41 & 0.51 \\
\hline \hline
\end{tabular}

This paper has been typeset from a $\mathrm{TEX}_{\mathrm{X}} / \mathrm{L} \mathrm{TEX}$ file prepared by the author. 